\title{
SOLITONS OF THE SINE-GORDON EQUATION COMING IN CLUSTERS
}

\author{
C. SCHIEBOLD *
}

\begin{abstract}
In the present paper, we construct a particular class of solutions of the sine-Gordon equation, which is the exact analogue of the so-called negatons, a solution class of the Korteweg-de Vries equation discussed by Matveev [17] and Rasinariu et al. [21]. Their characteristic properties are:

Each solution consists of a finite number of clusters.

Roughly speaking, in such a cluster solitons are grouped around a center, and the distance between two of them grows logarithmically. The clusters themselves rather behave like solitons. Moving with constant velocity, they collide elastically with the only effect of a phaseshift.

The main contribution of this paper is the proof that all this - including an explicit calculation of the phase-shift - can be expressed by concrete asymptotic formulas, which generalize very naturally the known expressions for solitons.

Our results confirm expectations formulated in the context of the Korteweg-de Vries equation by Matveev [17] and Rasinariu et al. [21].
\end{abstract}

\section{Introduction}

Soliton equations are a class of nonlinear evolution equations, which are usually qualified as integrable. Although there is still no satisfying general definition of integrability (see also the discussion in Zakharov [27]), one has a good intuition of the characteristic properties of integrability:

${ }^{*}$ Research supported by the Deutsche Forschungsgemeinschaft DFG

2000 Mathematics Subject Classification: 15A90, 35Q51, 35Q53, 37K40.

Servicio de Publicaciones. Universidad Complutense. Madrid, 2002 
(1) There are enough constants of the motion to rule out chaotic behaviour.

(2) A systematic solution theory is available: On the one hand one knows a large class of explicit solutions, whose behaviour reflect the essential properties of the system, on the other hand there is a general access to the initial value problem (inverse scattering method).

(3) There is an intuitive law, referred to as nonlinear superposition, how to construct solutions by combining known solutions. Physically, this corresponds to the fact that solitary waves interact without changing shape and speed.

Since the results of this article can be best motivated starting from (3), we want to take a closer look at the notion of superposition. For linear differential equations, it is just the trivial fact that sums of solutions are again solutions. Typically, this leads to the possibility to solve the initial value problem by integral formulas (superposition of fundamental solutions).

In the nonlinear case, which is more subtle, superposition is best understood by the example of Hirota's $N$-solitons [9]. Qualitatively, $N$ solitons describe the interaction of $N$ single solitary waves, each moving with constant velocity and permanent shape. Unlike as in the linear case, we have no precise addition. But it is almost precise (if we take into account the additional effect of a phase-shift), and it can in fact be shown that the $N$-soliton tends asymptotically for $t \rightarrow \pm \infty$ to a sum of $N$ single solitons (cf. Toda [26]).

The main goal of the present article is the complete asymptotic description of a class of examples, which show the following new type of nonlinear superposition: Again there is a finite number of solitary waves, but now those are coupled together in clusters. The clusters themselves move with constant velocity, whereas solitary waves contained in the very same cluster move away from each other only with a speed of $\mathcal{O}(\log |t|)$. (For a detailed presentation of the main result, we refer to the next section.)

The solution class described above was discovered 1992 by Matveev [14] in connection with the Darboux transformation, which was worked 
out by Matveev [13], too. He called them negatons in contrast to the analogous class of positons. The terminology is related to special properties relevant to the inverse scattering transform.

In subsequent articles (see also Beutler [3] for the situation most closely related to this article), Matveev [15], [16], [17] investigated the asymptotic behaviour of positons. In [17] he gave the asymptotic description of finite superpositions of solitons and positons of the lowest degree of complexity (see the last remark in Section 2 for some more details). In our context, there is also a significant contribution of Rasinariu et al. [21], where special cases of negatons of low complexity are examined.

In both cases ([17], [21]) natural expectations are formulated by the authors (in [17] for positons, in [21] for negatons). Our results settle completely the case of negatons.

We decided to present our result in the context of the sine-Gordon equation (see the author's thesis [22] for the Korteweg-de Vries equation) for the following two reasons: Firstly because in this setting negatons are regular and real-valued. This is not true e.g. for the Kortweg-de Vries equation, where clusters of higher order necessarily contain poles. Secondly because the structure of clusters is richer in the case of the sine-Gordon equation, where kinks, antikinks, and breathers participate in clusters, whereas for the Korteweg-de Vries equation only solitons and antisolitons appear.

The picture would not be complete without the sources of our main techniques. In contrast to the Darboux transformation of Matveev, we make use of explicit solution formulas which were discovered in an attempt to apply methods of functional analysis to soliton equations. Building upon pioneering work of Marchenko [12], Aden/Carl [1] deduced an explicit solution formula for the Korteweg-de Vries equation formulated in terms of determinants on operator ideals. The formula for the sine-Grodon equation we use in what follows was found in the author's thesis [22].

The paper is organized as follows.

The main result of this paper (Theorem C) is stated in Section 2 together with an exhaustive discussion of its geometric content. In Section 3 we give a self-contained proof of the solution formula for the sine- 
Gordon equation restricting all arguments to the finite dimensional situation. Section 4 contains the linear algebra part of the proof of Theorem C. To this end a series of simplifications and reductions of the hypothesis is performed. Some remaining questions about well-definedness are discussed in Section 5. In Section 6 we carry out the asymptotic analysis and finish the proof of Theorem C.

Finally, several plots are included to illustrate the result in Section 7. They were produced with Mathematica 3.0 at the PC-pool of the Fakultätsrechenzentrum of the Friedrich-Schiller-Universität Jena.

\section{Acknowledgment}

This paper was written within the scope of the project 'Methoden der Geometrischen Analysis und der Funktionalanalysis bei der Behandlung von nichtlinearen Gleichungen der Solitonentheorie' of the Deutsche Forschungsgemeinschaft. I am grateful to its director Professor B. Carl for constant support and fruitful discussions.

Moreover, I would like to thank the referee for valuable hints which helped to improve the overall quality of the paper considerably.

\section{Statement and discussion of the main results}

For the construction of $\mathrm{N}$-clusters of solitons, we exploit a general device originating from functional analysis. In the recent work [6], it was explained how the concept of operator ideals can be used to investigate soliton equations. The principle of this approach is due to Marchenko [12]. From a somewhat different point of view it was developed within the scope of operator ideals by Aden/Carl [1].

In the same spirit the author of the present paper obtained in [22] a general solution formula for the sine-Gordon equation

$$
u_{x t}=\sin (u)
$$

Restriction to the finite dimensional setting yields the following description of $N$-clusters of solitons:

Theorem A. Let $A: \mathbf{C}^{d} \longrightarrow \mathbf{C}^{d}$ be an arbitrary $d \times d$-matrix with $0 \notin \operatorname{spec}(A)+\operatorname{spec}(A)$. 
a) Then the elementary operator $\Phi_{A}: \mathcal{M}_{d, d}(\mathbf{C}) \longrightarrow \mathcal{M}_{d, d}(\mathbf{C})$ given by $\Phi_{A}(X)=A X+X A$ is invertible.

b) If we define $p_{ \pm}(x, t):=\operatorname{det}(1 \pm \mathrm{i} L(x, t))$ with $L(x, t)=\exp (A x+$ $\left.A^{-1} t\right) \Phi_{A}^{-1}(f \otimes \hat{f})$ for $f, \hat{f} \in \mathbf{C}^{d}$, then

$$
u(x, t)=2 \mathrm{i} \log \frac{p_{-}(x, t)}{p_{+}(x, t)}
$$

is a solution of the sine-Gordon equation.

Let us immediately state the following trivial but crucial lemma, which allows to restrict our attention to matrices in Jordan form.

Lemma B. Let $u(x, t)$ be any solution in Theorem $A$ generated by a matrix $A$, and $U$ the transformation of $A$ in Jordan canonical form $J$, $A=U^{-1} J U$. Then the following reformulation holds

$$
\begin{gathered}
u(x, t)=2 \mathrm{i} \log \frac{q_{-}(x, t)}{q_{+}(x, t)} \\
q_{ \pm}(x, t)=\operatorname{det}\left(1 \pm \mathrm{i} \exp \left(J x+J^{-1} t\right) \Phi_{J}^{-1}\left(\left(U^{-1}\right)^{t} f \otimes U \hat{f}\right)\right) .
\end{gathered}
$$

Next we observe that our solution class (2.2) comprises the $N$-solitons. Indeed, with a diagonal matrix

$A=\left(\begin{array}{cccc}a_{1} & 0 & \cdots & 0 \\ 0 & a_{2} & \cdots & 0 \\ \cdots & \cdots & \cdots & \cdots \\ 0 & 0 & \cdots & a_{d}\end{array}\right), \quad a_{j} \in \mathbf{C}$ all different and $a_{j}+a_{j^{\prime}} \neq 0\left(\forall j, j^{\prime}\right)$,

Theorem A yields

$$
p_{ \pm}(x, t)=
$$

$$
1+\sum_{k=1}^{d}( \pm \mathrm{i})^{k} \sum_{i_{1}<\ldots<i_{k}} \prod_{j=1}^{k}\left(\frac{f_{i_{j}} \hat{f}_{i_{j}}}{2 a_{i_{j}}} \exp \left(a_{i_{j}} x+a_{i_{j}}^{-1} t\right)\right) \prod_{j^{\prime}=j+1}^{k}\left(\frac{a_{i_{j}}-a_{i_{j^{\prime}}}}{a_{i_{j}}+a_{i_{j^{\prime}}}}\right)^{2} .
$$

The $N$-soliton solutions of Hirota [9] are obtained for

$$
\Re\left(a_{j}\right)>0 \quad \text { and } \quad \sum_{j=1}^{d} \frac{f_{j} \hat{f}_{j}}{2 a_{j}} \exp \left(a_{j} x+a_{j}^{-1} t\right) \in \mathbf{R}
$$


(for the proof see Pöppe [19]). Since $\overline{p_{+}(x, t)}=p_{-}(x, t)$, the solution can be rewritten as

$$
u(x, t)=-4 \arctan \frac{\Im\left(p_{-}(x, t)\right)}{\Re\left(p_{-}(x, t)\right)} .
$$

We emphasize that the dimension $d$ differs from the number $N$ of the solitons. In fact, one-solitons may arise in two different ways, either by one real eigenvalue or by a pair of complex conjugate eigenvalues. We recall these two basic configurations, which are the building blocks of the clusters.

If $N=1$, condition (2.4) tells that we get one-solitons only for

$$
a_{1}>0 \quad \text { and } \quad \frac{f_{1} \hat{f}_{1}}{2 a_{1}} \in \mathbf{R} .
$$

Writing $b:=a_{1}$ and $\left(f_{1} \hat{f}_{1}\right) /\left(2 a_{1}\right)=\epsilon \exp (\beta), \epsilon= \pm 1, \beta \in \mathbf{R}$, we get

$$
u_{b}(x, t)=4 \arctan \left(\epsilon \exp \left(b x+b^{-1} t+\beta\right)\right) .
$$

For short, (2.6) will be referred to as soliton for $\epsilon=1$, and antisoliton for $\epsilon=-1$.

If $N=2$, a different type of one-solitons is obtained for a pair of complex conjugate eigenvalues $a_{1}, a_{2}=\overline{a_{1}}$. In this situation, (2.4) yields

$$
\Re\left(a_{1}\right)>0 \quad \text { and } \quad \frac{f_{2} \hat{f}_{2}}{2 a_{2}}=\frac{\overline{f_{1} \hat{f}_{1}}}{2 a_{1}} .
$$

With $c:=a_{1}$ and $\left(f_{1} \hat{f}_{1}\right) /\left(2 a_{1}\right)=\exp (\gamma), \gamma \in \mathbf{C}$, we find

$$
u_{c}(x, t)=4 \arctan \left(\frac{1}{\alpha} \frac{\cos \left(\Im(c)\left[x-\frac{1}{|c|^{2}} t\right]+\Im(\gamma)\right)}{\cosh \left(\Re(c)\left[x+\frac{1}{|c|^{2}} t\right]+\Re(\gamma)+\log \alpha\right)}\right) \text {, }
$$

$\alpha=\frac{|\Im(c)|}{\Re(c)}$. As in the literature, (2.7) is called pulsating soliton.

For a geometric description, we turn to the sine-Gordon equation in the coordinates $\xi=x+t, \tau=-x+t$,

$$
u_{\tau \tau}-u_{\xi \xi}+\sin (u)=0
$$


In this form it applies to various physical problems, for example as a non-trivial model in field theory.

In the new coordinates, the soliton (2.6) becomes

$$
u_{b}(\xi, \tau)=4 \arctan \left(\epsilon \exp \left(\frac{\xi-\mu \tau}{\sqrt{1-\mu^{2}}}+\beta\right)\right),
$$

where $\mu=-\frac{1-b^{2}}{1+b^{2}}$. Thus, its velocity is $\mu$ with $-1<\mu<1$. In particular, there are stationary solitons, a feature specific to the sine-Gordon equation. Since its graph is similar to a step function, it is also called kink $(\epsilon=+1)$ or antikink $(\epsilon=-1)$. Let us remark that the quantity $\varphi:=-\sqrt{1-\mu^{2}} \beta$, usually denoted as the phase of the soliton, represents the coordinate of its center at $\tau=0$.

The pulsating soliton (2.7) now writes

$$
u_{c}(\xi, \tau)=4 \arctan \left(\frac{1}{\alpha} \frac{\cos \left(\omega \frac{\nu \xi-\tau}{\sqrt{1-\nu^{2}}}+\Im(\gamma)\right)}{\cosh \left(\sqrt{1-\omega^{2}} \frac{\xi-\nu \tau}{\sqrt{1-\nu^{2}}}+\Re(\gamma)+\log (\alpha)\right)}\right),
$$

where $c=|c|\left(\sqrt{1-\omega^{2}}+\mathrm{i} \omega\right), \alpha=\frac{|\omega|}{\sqrt{1-\omega^{2}}}$, and $\nu=-\frac{1-|c|^{2}}{1+|c|^{2}}$. Hence it moves with velocity $\nu,-1<\nu<1$, and its shape, varying because of the oscillating cosine term in (2.10), has motivated the notion breather. Remark that $\psi:=-\sqrt{1-\nu^{2}} \Re(\gamma)$ is the phase of the pulsating soliton.

In general, the $N$-soliton solution consists of $N$ such basic configurations as described above. Namely, an $N$-soliton is induced by a diagonal matrix

$$
A=\operatorname{diag}\left\{b_{1}, \ldots, b_{n}, c_{1}, \ldots, c_{m}, \overline{c_{1}}, \ldots, \overline{c_{m}}\right\}
$$

with

$$
b_{j}>0 \quad \forall j \quad \text { and } \quad \Re\left(c_{j^{\prime}}\right)>0 \quad \forall j^{\prime} .
$$

Then the resulting formula (2.2) describes the collision of $n$ solitons/antisolitons and $m$ pulsating solitons, thus $N=n+m$. As a remark, here the dimension $d$ equals $n+2 m$.

In other words, $N$-solitons are characterized by the fact that, for large times $\tau \rightarrow \pm \infty$, they look like a superposition of $n$ solitons/antisolitons and $m$ pulsating solitons $(N=n+m)$. In a mathematically precise way, this is formulated in terms of their asymptotic behaviour. 
Definition. The functions $u(\xi, \tau), v(\xi, \tau)$ possess the same asymptotic behaviour for $\tau \rightarrow \infty(\tau \rightarrow-\infty)$,

$$
u(\xi, \tau) \approx v(\xi, \tau) \quad \text { for } \tau \approx \infty(\tau \approx-\infty),
$$

if for every $\epsilon>0$ there is a $\tau_{\epsilon}$ such that, for $\tau>\tau_{\epsilon}\left(\tau<\tau_{\epsilon}\right), \mid u(\xi, \tau)-$ $v(\xi, \tau) \mid<\epsilon$ holds uniformly in $\xi$.

For $N$-solitons the asymptotic behaviour is well-known (Novikov [18]):

Result. Let the matrix $A$ in Theorem $A$ be given as in (2.11), (2.12), and assume $b_{j}, j=1, \ldots, n$, and $\left|c_{j^{\prime}}\right|, j^{\prime}=1, \ldots, m$, to be all different. We associate to this data

$$
\begin{gathered}
u_{b_{j}}^{ \pm}(\xi, \tau)=4 \arctan \left(\epsilon_{j} \exp \left(\frac{\xi-\mu_{j} \tau}{\sqrt{1-\mu_{j}^{2}}}+\left(\beta_{j}+\beta_{j}^{ \pm}\right)\right)\right), \\
u_{c_{j^{\prime}}}^{ \pm}(\xi, \tau)= \\
4 \arctan \left(\frac{1}{\alpha_{j^{\prime}}} \frac{\cos \left(\omega_{j^{\prime}} \frac{\nu_{j^{\prime}} \xi-\tau}{\sqrt{1-\nu_{j^{\prime}}^{2}}}+\Im\left(\gamma_{j^{\prime}}+\gamma_{j^{\prime}}^{ \pm}\right)\right)}{\cosh \left(\sqrt{1-\omega_{j^{\prime}}^{2}} \frac{\xi-\nu_{j^{\prime}} \tau}{\sqrt{1-\nu_{j^{\prime}}^{2}}}+\Re\left(\gamma_{j^{\prime}}+\gamma_{j^{\prime}}^{ \pm}\right)+\log \left(\alpha_{j^{\prime}}\right)\right)}\right),
\end{gathered}
$$

with $\mu_{j}=-\frac{1-b_{j}^{2}}{1+b_{j}^{2}}$ and $\left(f_{j} \hat{f}_{j}\right) /\left(2 b_{j}\right)=\epsilon_{j} \exp \left(\beta_{j}\right), \epsilon_{j}= \pm 1, \beta_{j} \in \mathbf{R}$, and $\nu_{j^{\prime}}=-\frac{1-\left|c_{j^{\prime}}\right|^{2}}{1+\left|c_{j^{\prime}}\right|^{2}}$ for $c_{j^{\prime}}=\left|c_{j^{\prime}}\right|\left(\sqrt{1-\omega_{j^{\prime}}^{2}}+\mathrm{i} \omega_{j^{\prime}}\right), \alpha_{j^{\prime}}=\left|\omega_{j^{\prime}}\right| / \sqrt{1-\omega_{j^{\prime}}^{2}}$, and $\left(f_{n+j^{\prime}} \hat{f}_{n+j^{\prime}}\right) /\left(2 c_{j^{\prime}}\right)=\exp \left(\gamma_{j^{\prime}}\right), \gamma_{j^{\prime}} \in \mathbf{C}$. The quantities $\beta_{j}^{ \pm}$and $\gamma_{j^{\prime}}^{ \pm}$ indicate a change of the phase in the asymptotic forms, namely

$$
\begin{gathered}
\beta_{j}^{ \pm}=\sum_{\substack{k=1 \\
\pm\left(b_{j}-b_{k}\right)>0}}^{n} \log \left(\frac{b_{k}-b_{j}}{b_{k}+b_{j}}\right)^{2}+\sum_{\substack{k^{\prime}=1 \\
\pm\left(b_{j}-c_{k^{\prime}} \mid\right)>0}}^{m} \log \left|\frac{c_{k^{\prime}}-b_{j}}{c_{k^{\prime}}+b_{j}}\right|^{4}, \\
\gamma_{j^{\prime}}^{ \pm}=\sum_{\substack{k=1 \\
\pm\left(\left|c_{j^{\prime}}\right|-b_{k}\right)>0}}^{n} \log \left(\frac{b_{k}-c_{j^{\prime}}}{b_{k}+c_{j^{\prime}}}\right)^{2}+\sum_{\substack{k^{\prime}=1 \\
\pm\left(\left|c_{j^{\prime}}\right|-\left|c_{k^{\prime}}\right|\right)>0}}^{m} \log \left[\left(\frac{c_{k^{\prime}}-c_{j^{\prime}}}{c_{k^{\prime}}+c_{j^{\prime}}}\right)\left(\frac{\overline{c_{k^{\prime}}}-c_{j^{\prime}}}{\overline{c_{k^{\prime}}}+c_{j^{\prime}}}\right)\right]^{2} .
\end{gathered}
$$


Then the asymptotic behaviour of the corresponding solution (2.2) in Theorem $A$ is described by

$$
u(\xi, \tau) \approx \sum_{j=1}^{n} u_{b_{j}}^{ \pm}(\xi, \tau)+\sum_{j^{\prime}=1}^{m} u_{c_{j^{\prime}}}^{ \pm}(\xi, \tau) \quad \text { for } \tau \approx \pm \infty .
$$

Therefore, one can visualize the wave $u_{b_{j}}$ (or $u_{c_{j^{\prime}}}$ ) as travelling with constant velocity for all times. As time goes by, collisions with the other participating waves happen, which are elastic and leave the shape of the wave $u_{b_{j}}\left(\right.$ or $\left.u_{c_{j^{\prime}}}\right)$ unchanged. The only effect caused by such a collision is a phase-shift.

Remark. a) The assumption that $b_{j}(j=1, \ldots, n),\left|c_{j^{\prime}}\right|\left(j^{\prime}=1, \ldots, m\right)$ are all different means that the waves move with different velocities.

By the way, our method also leads to solutions where several waves travel with the same velocity (for example a soliton and a pulsating soliton, both stationary). In the present work we shall not persue the study of these degeneracies.

b) Further asymptotic results related to the inverse scattering method are proved in Schuur [24].

Up to now we have seen how simple eigenvalues lead to solitons. We shall next explain that larger Jordan blocks produce clusters.

Before we give the complicated formulation of our main theorem, we first shall discuss its geometric content.

\section{Interpretation.}

a) To start with, we consider the case of a single Jordan block $B_{1}$ of dimension $n_{1}$ corresponding to an eigenvalue $b_{1}>0$. The resulting solution consists of a wavepacket - a cluster - containing $n_{1}$ waves each of them being either a soliton or an antisoliton (their shapes are identical depending only on the eigenvalue $b_{1}$ ).

The first observation is that the geometric center of the cluster propagates with constant velocity $\mu_{1}=-\frac{1-b_{1}^{2}}{1+b_{1}^{2}}$ whereas the involved solitons/antisolitons themselves drift apart at most logarithmically.

Now, for large negative times $\tau<<0$, one can imagine each soliton/antisoliton to be located on one side of the center, approaching the center logarithmically. Sometime it changes over to the other side of 
the center (colliding elastically with other waves of the cluster in the meantime). For large positive times $\tau>>0$, the soliton/antisoliton thus is located on the opposite side, moving away from the center again logarithmically.

According to this interpretation - which is motivated by computer experiments - the solitons/antisolitons appear exactly in reversed order in the asymptotic forms for $-\infty$ and $+\infty$. As a matter of fact, solitons and antisolitons always alternate. In particular, there are only two different types of asymptotic forms depending on whether the first involved wave in the asymptotic form for $-\infty$ is a soliton or an antisoliton. The latter is determined by the sign $\epsilon_{1}$ of the parameter responsible for the phase of the cluster.

It is remarkable that the cluster itself, or, more precisely, the path of its geometric center, is not disturbed by internal collisions.

b) Next we consider the case that the solution belongs to two Jordan blocks $C_{1}, \overline{C_{1}}$, both of dimension $m_{1}$, corresponding to a pair of complex conjugate eigenvalues $c_{1}, \overline{c_{1}}$ with $\Re\left(c_{1}\right)>0$. Again the solution forms a cluster, now containing $m_{1}$ pulsating solitons (with identical shapes depending only on the eigenvalue $c_{1}$ ).

The geometric center of the cluster propagates with velocity $\nu_{1}=$ $-\frac{1-\left|c_{1}\right|^{2}}{1+\left|c_{1}\right|^{2}}$, and again the involved pulsating solitons drift apart with logarithmic speed. As in a), one visualizes each pulsating soliton on one side of the center. As time goes by, the pulsating solitons get closer, collide, and separate again. Once more, the internal collisions do not effect the center.

In the asymptotic forms for $\pm \infty$, now only pulsating solitons appear. However, the statement that solitons and antisolitons always alternate remains true if we take into account that each pulsating soliton can be considered as a bound state of a soliton and an antisoliton. The latter was shown in Novikov et al., see [18] for details.

Let us in addition point out that the oscillation of the pulsating solitons is synchronized in the asymptotic forms for $\pm \infty$.

c) In general, a solution consists of $N=n+m$ clusters,

- $n$ clusters of solitons/antisolitons as described in a),

( $n$ the number of Jordan blocks $B_{j}$ of dimension $n_{j}$ with eigenvalue $b_{j}>0$ ), 
- $m$ clusters of pulsating solitons as described in $\mathbf{b})$,

( $m$ the number of pairs of Jordan blocks $C_{j^{\prime}}, \overline{C_{j^{\prime}}}$ of dimension $m_{j^{\prime}}$ with complex conjugate eigenvalues $c_{j^{\prime}}, \overline{c_{j^{\prime}}}$, where $\left.\Re\left(c_{j^{\prime}}\right)>0\right)$.

As a whole, clusters rather behave like solitons. They collide elastically and the only effect of the collision is a phase-shift (of the whole cluster). Moreover, the resulting phase-shifts $\beta_{j}^{ \pm}$for the $j$-th cluster of solitons/antisolitons, see (2.26), and $\Re\left(\gamma_{j^{\prime}}^{ \pm}\right)$for the $j^{\prime}$-th cluster of pulsating solitons, see (2.27), are a very natural extension of the formulas for the $N$-soliton, see (2.15), (2.16), from which they differ only by the exponents $n_{j}, m_{j^{\prime}}$, respectively.

The following theorem is the main achievement of the present article and translates the preceeding interpretation into exact formulas.

Theorem C. Let the matrix $A$ in Theorem $A$ be given by

$$
A=\operatorname{diag}\left\{B_{1}, \ldots, B_{n}, C_{1}, \ldots, C_{m}, \overline{C_{1}}, \ldots, \overline{C_{m}}\right\},
$$

$B_{j}$ Jordan block of dimension $n_{j}$ corresponding to the eigenvalue $b_{j}$ $(j=1, \ldots, n)$,

$C_{j^{\prime}}, \overline{C_{j^{\prime}}}$ Jordan blocks of dimension $m_{j^{\prime}}$ corresponding to a pair of complex conjugate eigenvalues $c_{j^{\prime}}, \overline{c_{j^{\prime}}}\left(j^{\prime}=1, \ldots, m\right)$,

$$
b_{j}>0 \quad \forall j \quad \text { and } \quad \Re\left(c_{j^{\prime}}\right)>0 \quad \forall j^{\prime} .
$$

Assume in addition $b_{j}, j=1, \ldots, n$, and $\left|c_{j^{\prime}}\right|, j^{\prime}=1, \ldots, m$, to be all different.

Adapted to this Jordan canonical form, we decompose the vectors $f, \hat{f} \in \mathbf{C}^{d}$ accordingly $f=\left(f_{1}, \ldots, f_{n+2 m}\right)^{t}$, and for $j=1, \ldots, n, j^{\prime}=$ $1, \ldots, m$,

$$
\begin{gathered}
f_{j}=\left(f_{j}^{(1)}, \ldots, f_{j}^{\left(n_{j}\right)}\right)^{t}, \quad f_{n+j^{\prime}}=\left(f_{n+j^{\prime}}^{(1)}, \ldots, f_{n+j^{\prime}}^{\left(m_{j^{\prime}}\right)}\right)^{t}, \\
f_{n+m+j^{\prime}}=\left(f_{n+m+j^{\prime}}^{(1)}, \ldots, f_{n+m+j^{\prime}}^{\left(m_{j^{\prime}}\right)},\right.
\end{gathered}
$$

with $\sum_{\kappa=1}^{n_{j}-\delta+1} f_{j}^{(\kappa)} \hat{f}_{j}^{(\kappa+\delta-1)} \in \mathbf{R}$, and $\sum_{\kappa^{\prime}=1}^{m_{j}-\delta^{\prime}+1} \overline{f_{n+m+j^{\prime}}^{\left(\kappa^{\prime}\right)} \hat{f}_{n+m+j^{\prime}}^{\left(\kappa^{\prime}+\delta^{\prime}-1\right)}}=$ $\sum_{\kappa^{\prime}=1}^{m_{j}-\delta^{\prime}+1} f_{n+j^{\prime}}^{\left(\kappa^{\prime}\right)} \hat{f}_{n+j^{\prime}}^{\left(\kappa^{\prime}+\delta^{\prime}-1\right)}\left(\delta=1, \ldots, n_{j}, \delta^{\prime}=1, \ldots, m_{j^{\prime}}\right)$. 
We associate to this data the curves

$$
\mathcal{B}_{b_{j}, k_{j}}(\xi, \tau)=\frac{\xi-\mu_{j} \tau}{\sqrt{1-\mu_{j}^{2}}}+\log |\tau|^{\mp k_{j}}+\left(\beta_{j}+\beta_{j}^{ \pm}+\beta_{j, k_{j}}^{ \pm}\right)
$$

$$
\text { for } k_{j}=-\left(n_{j}-1\right),-\left(n_{j}-1\right)+2, \ldots,\left(n_{j}-1\right)-2,\left(n_{j}-1\right) \text {, }
$$

$$
\begin{aligned}
\mathcal{C}_{c_{j^{\prime}}, \kappa_{j^{\prime}}}(\xi, \tau)= & \sqrt{1-\omega_{j^{\prime}}^{2}} \frac{\xi-\nu_{j^{\prime}} \tau}{\sqrt{1-\nu_{j^{\prime}}^{2}}}+\log |\tau|^{\mp \kappa_{j^{\prime}}}+\mathrm{i} \omega_{j^{\prime}} \frac{\nu_{j^{\prime}} \xi-\tau}{\sqrt{1-\nu_{j^{\prime}}^{2}}} \\
& +\left(m_{j^{\prime}} \pm \kappa_{j^{\prime}}\right) \log \left(\alpha_{j^{\prime}}\right)+\left(\gamma_{j^{\prime}}+\gamma_{j^{\prime}}^{ \pm}+\gamma_{j^{\prime}, \kappa_{j^{\prime}}}^{ \pm}\right),
\end{aligned}
$$

for $\kappa_{j^{\prime}}=-\left(m_{j^{\prime}}-1\right),-\left(m_{j^{\prime}}-1\right)+2, \ldots,\left(m_{j^{\prime}}-1\right)-2,\left(m_{j^{\prime}}-1\right),(2.23)$ and, along this curves, the solitons

$$
\begin{aligned}
& u_{b_{j}, k_{j}}^{ \pm}(\xi, \tau)= \\
& (\mp 1)^{\left(n_{j}-1\right)} 4 \arctan \left((-1)^{\frac{\left(n_{j}-1\right)+k_{j}}{2}} \epsilon_{j} \exp \left(\mathcal{B}_{b_{j}, k_{j}}(\xi, \tau)\right)\right), \\
& u_{c_{j^{\prime}, \kappa_{j^{\prime}}}}^{ \pm}(\xi, \tau)= \\
& \quad(\mp 1)^{\left(m_{j^{\prime}}-1\right)} 4 \arctan \left(\frac{1}{\alpha_{j^{\prime}}} \frac{\cos \left(\Im\left(\mathcal{C}_{c_{j^{\prime}}, \kappa_{j^{\prime}}}(\xi, \tau)\right)\right)}{\cosh \left(\Re\left(\mathcal{C}_{c_{j^{\prime}}, \kappa_{j^{\prime}}}(\xi, \tau)\right)\right)}\right),
\end{aligned}
$$

with the notations $\mu_{j}=-\frac{1-b_{j}^{2}}{1+b_{j}^{2}}$ and $\left(f_{j}^{(1)} \hat{f}_{j}^{\left(n_{j}\right)}\right) /\left(2 b_{j}\right)^{n_{j}}=\epsilon_{j} \exp \left(\beta_{j}\right)$, $\epsilon_{j}= \pm 1, \beta_{j} \in \mathbf{R}$, and $\nu_{j^{\prime}}=-\frac{1-\left|c_{j^{\prime}}\right|^{2}}{1+\left|c_{j^{\prime}}\right|^{2}}$ for $c_{j^{\prime}}=\left|c_{j^{\prime}}\right|\left(\sqrt{1-\omega_{j^{\prime}}^{2}}+\mathrm{i} \omega_{j^{\prime}}\right)$, $\alpha_{j^{\prime}}=\left|\omega_{j^{\prime}}\right| / \sqrt{1-\omega_{j^{\prime}}^{2}}$, and $\left(f_{n+j^{\prime}}^{(1)} \hat{f}_{n+j^{\prime}}^{\left(m_{j^{\prime}}\right)}\right) /\left(2 c_{j^{\prime}}\right)^{m_{j^{\prime}}}=\exp \left(\gamma_{j^{\prime}}\right), \gamma_{j^{\prime}} \in \mathbf{C}$.

The quantities $\beta_{j}^{ \pm}, \beta_{j, k_{j}}^{ \pm}$and $\gamma_{j^{\prime}}^{ \pm}, \gamma_{j^{\prime}, \kappa_{j^{\prime}}}^{ \pm}$indicate a change of the phase in the asymptotic forms, namely

$$
\beta_{j}^{ \pm}=\sum_{\substack{k=1 \\ \pm\left(b_{j}-b_{k}\right)>0}}^{n} \log \left(\frac{b_{k}-b_{j}}{b_{k}+b_{j}}\right)^{2 n_{k}}+\sum_{\substack{k^{\prime}=1 \\ \pm\left(b_{j}-\left|c_{k^{\prime}}\right|\right)>0}}^{m} \log \left|\frac{c_{k^{\prime}}-b_{j}}{c_{k^{\prime}}+b_{j}}\right|^{4 m_{k^{\prime}}},
$$




$$
\begin{gathered}
\gamma_{j^{\prime}}^{ \pm}=\sum_{\substack{k=1 \\
\pm\left(\left|c_{j^{\prime}}\right|-b_{k}\right)>0}}^{n} \log \left(\frac{b_{k}-c_{j^{\prime}}}{b_{k}+c_{j^{\prime}}}\right)^{2 n_{k}}+\sum_{\substack{k^{\prime}=1 \\
\pm\left(\left|c_{j^{\prime}}\right|-\left|c_{k^{\prime}}\right|\right)>0}}^{m} \log \left[\left(\frac{c_{k^{\prime}}-c_{j^{\prime}}}{c_{k^{\prime}}+c_{j^{\prime}}}\right)\left(\frac{\overline{c_{k^{\prime}}}-c_{j^{\prime}}}{\overline{c_{k^{\prime}}}+c_{j^{\prime}}}\right)\right]^{2 m_{k^{\prime}}} \\
\beta_{j, k_{j}}^{ \pm}=\log \frac{\left(\frac{\left(n_{j}-1\right) \pm k_{j}}{2}\right) !}{\left(\frac{\left(n_{j}-1\right) \mp k_{j}}{2}\right) !}\left(\frac{4 b_{j}}{1+b_{j}^{2}}\right)^{\mp k_{j}} \\
\gamma_{j^{\prime}, \kappa_{j^{\prime}}}^{ \pm}=\log \frac{\left(\frac{\left(m_{j^{\prime}}-1\right) \pm \kappa_{j^{\prime}}}{2}\right) !}{\left(\frac{\left(m_{j^{\prime}}-1\right) \mp \kappa_{j^{\prime}}}{2}\right) !}\left(\frac{4 \Re\left(c_{j^{\prime}}\right)}{1+\left|c_{j^{\prime}}\right|^{2}}\right)^{\mp \kappa_{j^{\prime}}}
\end{gathered}
$$

Then the asymptotic behaviour of the corresponding solution (2.2) in Theorem $A$ is described by

$$
u(\xi, \tau) \approx \sum_{j=1}^{n} \sum_{k_{j}} u_{b_{j}, k_{j}}^{ \pm}(\xi, \tau)+\sum_{j^{\prime}=1}^{m} \sum_{\kappa_{j^{\prime}}} u_{c_{j^{\prime}}, \kappa_{j^{\prime}}}^{ \pm}(\xi, \tau) \quad \text { for } \tau \approx \pm \infty
$$

(the summation indices $k_{j}$ given by (2.21), $\kappa_{j^{\prime}}$ by (2.23)), and hence, the following equalities for the dimension $d$ and the number $N$ of participating solitons hold

$$
d=\sum_{j=1}^{n} n_{j}+2 \sum_{j^{\prime}=1}^{m} m_{j^{\prime}}, \quad N=\sum_{j=1}^{n} n_{j}+\sum_{j^{\prime}=1}^{m} m_{j^{\prime}} .
$$

Let us stress that our results concerning asymptotics are a complete generalization of what is known for $N$-solitons. In our opinion, this proves how nicely adapted the geometric background of Theorem A is to the dynamical nature of our solution class.

Taking into consideration the interpretation of pulsating solitons as bound states of a soliton and an antisoliton (see Novikov et al. [18]), we derive the following conservation law as an immediate consequence of Theorem C. 
Corollary. The sum over all phase-shifts vanishes:

$$
\sum_{j=1}^{n} n_{j}\left(\beta_{j}^{+}-\beta_{j}^{-}\right)+2 \sum_{j^{\prime}=1}^{m} m_{j^{\prime}}\left(\Re\left(\gamma_{j^{\prime}}^{+}\right)-\Re\left(\gamma_{j^{\prime}}^{-}\right)\right)=0 .
$$

Moreover, we can calculate the 'topological charge' $Q$,

$$
Q=\frac{1}{2 \pi} \int_{-\infty}^{\infty} u_{\xi}(\xi, \tau) d \xi
$$

which is evidently a conserved quantity for (2.8) (see e. g. [18]). For a soliton/antisoliton, $Q=\epsilon$, whereas a pulsating soliton - as a bound state of a soliton and an antisoliton - contributes $Q=0$. This generalizes naturally to the solutions described in Theorem $\mathrm{C}$.

Corollary. $\quad Q=\sum_{\substack{j=1 \\ n_{j} \text { odd }}}^{n} \epsilon_{j}$.

Finally we should mention some further literature, which is most closely related to the subject of this article.

Remark. a) The solutions discussed above were already mentioned by Pöppe [19]. He used the notion 'multipole solution' (for a single cluster) since it corresponds to scattering data with a multiple pole in the reflection coefficient.

However, Pöppe did not treat the asymptotic behaviour of "multipole solutions".

Recently, the concept of positons was introduced by Matveev in [14] in connection with the Korteweg-de Vries equation, for a more detailed version see [17] as well. The sine-Gordon equation was considered by Beutler [3].

Roughly speaking, positons of order $N$ arise by eigenvalues $a_{j}(j=$ $1, \ldots, 2 N)$ which are purely imaginary by taking the limit $a_{j} \rightarrow a$, $a \in \mathrm{iR}$, for all $j=1, \ldots, 2 N$. These solutions are weakly localized, oscillating at infinity, and decay like $1 / \xi$. Moreover, in positon-positon collisions no phase-shifts occur at all.

The difference to the behaviour of a single cluster - not considered by Matveev et al. - is remarkable, particularly with regard to the fact 
that it can be constructed by essentially the same procedure. Namely, choosing positive eigenvalues $a_{j}(j=1, \ldots, N)$ and taking the limit for $a_{j} \rightarrow a, a>0$, for all $j=1, \ldots, N$, we end up with a single cluster, a negaton of order $N$ in the notion of Matveev et al..

(The interpretation of positons of order $N$ as limit of $2 N$-solitons is due to Jaworski et al. [10].)

c) The smallest interesting case, $N=2$, is studied in detail in [23] for the Toda lattice equation. For the sine-Gordon equation, the corresponding solution for $N=2$ is also noted in [5], [11].

In several articles [7], [8], Gesztesy et al. treated the very natural problem how to obtain solutions as limits of $N$-solitons as $N$ tends to infinity. For the greater class of $N$-clusters of solitons we shall discuss the analogous question in a subsequent article.

\section{Explicit construction of solution formulas}

In this section, we give the proof of Theorem A.

For the sake of readability, we restrict all considerations to the finite dimensional situation. Nevertheless we want to stress that all arguments extend to the much more general context of quasi-Banach operator ideals (see [22]).

Moreover, there is a connection between the so-called bilinear method of Hirota [9] and Theorem A. To be precise, it can be shown that the functions $p_{+}(x, t), p_{-}(x, t)$ as defined in Theorem $\mathrm{A}$ are a solution of the 'Sym equation', the bilinear form of the sine-Gordon equation (this is proved as well in [22]). Then the solution $u(x, t)$ of Theorem $\mathrm{A}$ is given by $u(x, t)=2 \mathrm{i} \log p_{-}(x, t) / p_{+}(x, t)$ which transforms the solution $p_{-}(x, t), p_{+}(x, t)$ of the Sym equation into a solution of the sine-Gordon equation.

(To be brief, here we will follow a different line of reasoning.)

Let us first treat a matrix sine-Gordon equation.

Proposition 3.1. Let $A \in \mathcal{M}_{d, d}(\mathbf{C})$ be an invertible $d \times d$-matrix over $\mathbf{C}$ and, for $B \in \mathcal{M}_{d, d}(\mathbf{C})$ arbitrary, $L(x, t)=\exp \left(A x+A^{-1} t\right) B$ a smooth 
family of matrices. Then

$$
\begin{aligned}
& V_{+}:=1-\mathrm{i}(1+\mathrm{i} L)^{-1}\left(A L A^{-1}+L\right) \\
& V_{-}:=1+\mathrm{i}(1-\mathrm{i} L)^{-1}\left(A L A^{-1}+L\right)
\end{aligned}
$$

yield a solution of the matrix sine-Gordon equation

$$
\frac{\partial}{\partial t}\left(\left(V_{-}^{-1} \frac{\partial}{\partial x} V_{-}\right)-\left(V_{+}^{-1} \frac{\partial}{\partial x} V_{+}\right)\right)=\frac{1}{2}\left(V_{+}^{-1} V_{-}-V_{-}^{-1} V_{+}\right),
$$

provided that $(1 \pm \mathrm{i} L)^{-1}$ exist.

Proof. By the so-called base equations

$$
\frac{\partial}{\partial x} L=A L \quad \text { and } \quad \frac{\partial}{\partial t} L=A^{-1} L,
$$

we easily calculate

$$
\begin{aligned}
& \frac{\partial}{\partial x} V_{ \pm}= \\
= & \pm \mathrm{i}(1 \pm \mathrm{i} L)^{-1}( \pm \mathrm{i} A L)(1 \pm \mathrm{i} L)^{-1}\left(A L A^{-1}+L\right) \mp \mathrm{i}(1 \pm \mathrm{i} L)^{-1} A\left(A L A^{-1}+L\right) \\
= & \mp \mathrm{i}(1 \pm \mathrm{i} L)^{-1} A(1 \pm \mathrm{i} L)^{-1}\left(A L A^{-1}+L\right)
\end{aligned}
$$

and

$$
\begin{aligned}
V_{ \pm}^{-1} & =\left((1 \pm \mathrm{i} L)^{-1}\left((1 \pm \mathrm{i} L) \mp \mathrm{i}\left(A L A^{-1}+L\right)\right)\right)^{-1} \\
& =\left((1 \pm \mathrm{i} L)^{-1} A(1 \mp \mathrm{i} L) A^{-1}\right)^{-1} \\
& =A(1 \mp \mathrm{i} L)^{-1} A^{-1}(1 \pm \mathrm{i} L) .
\end{aligned}
$$

Thus, on the one hand we get

$$
\begin{aligned}
\mp \frac{\partial}{\partial t} & \left(V_{ \pm}^{-1} \frac{\partial}{\partial x} V_{ \pm}\right)=\mathrm{i} \frac{\partial}{\partial t}\left(A\left(1+L^{2}\right)^{-1}\left(A L A^{-1}+L\right)\right) \\
& =-\mathrm{i} A\left(1+L^{2}\right)^{-1}\left(A^{-1} L^{2}+L A^{-1} L\right)\left(1+L^{2}\right)^{-1}\left(A L A^{-1}+L\right) \\
& +\mathrm{i} A\left(1+L^{2}\right)^{-1} A^{-1}\left(A L A^{-1}+L\right) \\
& =\mathrm{i} A\left(1-L^{2}\right)^{-1}\left(-\left(A^{-1} L^{2}+L A^{-1} L\right)+A^{-1}\left(1+L^{2}\right)\right)\left(1+L^{2}\right)^{-1}\left(A L A^{-1}+L\right) \\
& =\mathrm{i} A\left(1+L^{2}\right)^{-1}\left(A^{-1}-L A^{-1} L\right)\left(1+L^{2}\right)^{-1}\left(A L A^{-1}+L\right) .
\end{aligned}
$$


On the other hand, from

$V_{+}-V_{-}=-\mathrm{i}\left((1-\mathrm{i} L)^{-1}+(1+\mathrm{i} L)^{-1}\right)\left(A L A^{-1}+L\right)=-2 \mathrm{i}\left(1+L^{2}\right)^{-1}\left(A L A^{-1}+L\right)$

and

$$
\begin{aligned}
V_{+}^{-1}+V_{-}^{-1} & =A\left(1+L^{2}\right)^{-1}\left((1+\mathrm{i} L) A^{-1}(1+\mathrm{i} L)+(1-\mathrm{i} L) A^{-1}(1-\mathrm{i} L)\right) \\
& =2 A\left(1+L^{2}\right)^{-1}\left(A^{-1}-L A^{-1} L\right)
\end{aligned}
$$

we conclude

$$
\begin{aligned}
& \left(V_{+}^{-1} V_{-}-V_{-}^{-1} V_{+}\right)=-\left(V_{+}^{-1}+V_{-}^{-1}\right)\left(V_{+}-V_{-}\right) \\
& \quad=4 \mathrm{i} A\left(1+L^{2}\right)^{-1}\left(A^{-1}-L A^{-1} L\right)\left(1+L^{2}\right)^{-1}\left(A L A^{-1}+L\right) .
\end{aligned}
$$

Remark. Extensions of the sine-Gordon equation to an analogous equation of higher (but finite) dimension have already attracted attention in the literature. For an example of such an equation in a form which is somehow similar to (3.2), see Andreev [2]. Commonly, the corresponding equation is interpreted as a system with a finite number of equations, and subsequently solution techniques such as the inverse scattering transform or, as in [2], Bäcklund transformations are developed for this system.

However, our approach is quite different: Reading (3.2) as a matrixvalued sine-Gordon equation, our solution (3.1) can be viewed as a matrix-valued generalization of the well-known one-soliton.

Next we prove Theorem A.

\section{Proof (of Theorem A)}

a) In the matrix case, the solvability of the equation $A X-X B=$ $Y$, where $A, B$, and $Y \in \mathcal{M}_{d, d}(\mathbf{C})$ are given matrices, was originally shown by Sylvester [25]. For the convenience of the reader, we give an elementary proof.

Evaluation of $\Phi_{A}$ at an arbitrary one-dimensional operator $w \otimes v$,

$$
\Phi_{A}(w \otimes v)=\left(A^{t} w\right) \otimes v+w \otimes(A v),
$$


immediately shows that $\lambda+\mu$ is an eigenvalue of $\Phi_{A}$ with eigenvector $w \otimes v$ whenever $\lambda$ is an eigenvalue of $A$ with eigenvector $v$ and $\mu$ is an eigenvalue of $A^{t}$ with eigenvector $w\left(\right.$ remember $\left.\operatorname{spec}(A)=\operatorname{spec}\left(A^{t}\right)\right)$.

To show

$$
\operatorname{spec}\left(\Phi_{A}\right)=\operatorname{spec}(A)+\operatorname{spec}(A),
$$

it is therefore sufficient to observe that the (algebraic) multiplicity of the eigenvalue $\lambda+\mu$ of $\Phi_{A}$ is not smaller than the product of the (algebraic) multiplicities of the eigenvalues $\lambda$ and $\mu$ of $A$.

To this end, let us denote by $\mathcal{N}(A, \lambda)$ the nullspace of a sufficiently high power of $A-\lambda \mathbf{1}$ whose sequence of nullspaces is stationary. Hence the algebraic multiplicity of the eigenvalue $\lambda$ equals the dimension of $\mathcal{N}(A, \lambda)$.

Similarly as above, we have

$$
\left(\Phi_{A}-(\lambda+\mu) \mathbf{1}\right)^{k}(w \otimes v)=\sum_{\kappa=0}^{k}\left(\begin{array}{l}
k \\
\kappa
\end{array}\right)\left(\left(A^{t}-\mu\right)^{\kappa} w\right) \otimes\left((A-\lambda)^{k-\kappa} v\right) .
$$

Thus, if $v_{1}, \ldots, v_{n}$ form a base of $\mathcal{N}(A, \lambda)$ and $w_{1}, \ldots, w_{m}$ a base of $\mathcal{N}\left(A^{t}, \mu\right)$, then the $n \cdot m$ matrices $w_{i} \otimes v_{j}(i=1, \ldots, m, j=1, \ldots, n)$ obviously are linearly independent in $\mathcal{N}\left(\Phi_{A}, \lambda+\mu\right)$. For dimensional reasons, this already yields the assertion.

b) First we observe that, given a solution $v_{-}, v_{+}$of the scalar version of equation (3.2), we obtain a solution $u$ of the sine-Gordon equation (2.1) by the transformation

$$
u=\mathrm{i} \log \frac{v_{-}}{v_{+}} .
$$

Next we exploit Proposition 3.1 to extract scalar solutions from matrix-valued solutions of equation (3.2).

According to a) we choose $B:=\Phi_{A}^{-1}(f \otimes \hat{f}) \cdot A$. Thus the solution given by Proposition 3.1 is $V_{ \pm}=1 \mp \mathrm{i}(1 \pm \mathrm{i} L)^{-1} L \cdot f \otimes \hat{f}$, and

$$
\begin{aligned}
& V_{\mp}^{-1} V_{ \pm}-1= \\
& \quad=V_{\mp}^{-1}\left(V_{ \pm}-V_{\mp}\right)=V_{\mp}^{-1}\left(\mp \mathrm{i}\left((1 \pm \mathrm{i} L)^{-1}+(1 \mp \mathrm{i} L)^{-1}\right) L \cdot f \otimes \hat{f}\right) \\
& \quad=\mp 2 \mathrm{i} V_{\mp}^{-1}\left(1+L^{2}\right)^{-1} L \cdot f \otimes \hat{f}
\end{aligned}
$$


is one-dimensional.

In preparation, let us recall the known relations

$\operatorname{det}(1+g \otimes \hat{g})=1+\operatorname{tr}(g \otimes \hat{g}) \quad$ for one-dimensional matrices $g \otimes \hat{g}$,

$$
\frac{\partial}{\partial x} \operatorname{det}(V)=\operatorname{det}(V) \cdot \operatorname{tr}\left(V^{-1} \frac{\partial}{\partial x} V\right) \text { for any } V \in \mathcal{M}_{d, d}(\mathbf{C}) .
$$

Now we show that the scalar functions $v_{ \pm}:=\operatorname{det}\left(V_{ \pm}\right)$are solutions of the scalar version of equation (3.2). Observe

$$
\begin{aligned}
\frac{1}{2}\left(\frac{v_{-}}{v_{+}}-\frac{v_{+}}{v_{-}}\right)= \\
\quad=\frac{1}{2}\left(\frac{\operatorname{det}\left(V_{-}\right)}{\operatorname{det}\left(V_{+}\right)}-\frac{\operatorname{det}\left(V_{+}\right)}{\operatorname{det}\left(V_{-}\right)}\right)=\frac{1}{2}\left(\operatorname{det}\left(V_{+}^{-1} V_{-}\right)-\operatorname{det}\left(V_{-}^{-1} V_{+}\right)\right) \\
\stackrel{(3.5)}{=} \frac{1}{2}\left(\left(1+\operatorname{tr}\left(V_{+}^{-1} V_{-}-1\right)\right)-\left(1+\operatorname{tr}\left(V_{-}^{-1} V_{+}-1\right)\right)\right) \\
\quad=\frac{1}{2} \operatorname{tr}\left(V_{+}^{-1} V_{-}-V_{-}^{-1} V_{+}\right) \\
\quad=\operatorname{tr}\left(\frac{\partial}{\partial t}\left(\left(V_{-}^{-1} \frac{\partial}{\partial x} V_{-}\right)-\left(V_{+}^{-1} \frac{\partial}{\partial x} V_{+}\right)\right)\right)(\text {by Proposition 3.1) } \\
\quad=\frac{\partial}{\partial t}\left(\operatorname{tr}\left(V_{-}^{-1} \frac{\partial}{\partial x} V_{-}\right)-\operatorname{tr}\left(V_{+}^{-1} \frac{\partial}{\partial x} V_{+}\right)\right) \\
\left.\quad \stackrel{\frac{\partial}{\partial 3.6)}}{=} \frac{\frac{\partial}{\partial x} \operatorname{det}\left(V_{-}\right)}{\operatorname{det}\left(V_{-}\right)}-\frac{\frac{\partial}{\partial x} \operatorname{det}\left(V_{+}\right)}{\operatorname{det}\left(V_{+}\right)}\right)=\frac{\partial}{\partial t}\left(\frac{\frac{\partial}{\partial x} v_{-}}{v_{-}}-\frac{\frac{\partial}{\partial x} v_{+}}{v_{+}}\right) .
\end{aligned}
$$

Since $f \otimes \hat{f}=A B A^{-1}+B$ by the choice of $B$, we finally get

$$
\begin{aligned}
v_{ \pm} & =\operatorname{det}\left(1 \mp \mathrm{i}(1 \pm \mathrm{i} L)^{-1}\left(A L A^{-1}+L\right)\right) \\
& =\operatorname{det}\left((1 \pm \mathrm{i} L)^{-1} A(1 \mp \mathrm{i} L) A^{-1}\right)=\frac{\operatorname{det}(1 \mp \mathrm{i} L)}{\operatorname{det}(1 \pm \mathrm{i} L)},
\end{aligned}
$$

which completes the proof.

Remark. The solvability of the equation $A X-X B=Y$, considered as an operator equation, is the subject of numerous publications, see [4] for further references and a comprehensive survey of applications. 


\section{Some preparational reductions}

The main problem in the study of the class of $N$-clusters is that in contrast to $N$-solitons it is no longer possible to calculate the determinants $p_{ \pm}(x, t)$ in Theorem A explicitely. Nevertheless, we can carry out an asymptotic analysis. To this end, in the present section we perform some reduction steps.

To begin with, we prove Lemma B, which allows to confine all considerations to matrices in Jordan form.

\section{Proof (of Lemma B)}

We immediately check $\Phi_{J}\left(U B U^{-1}\right)=U\left(\Phi_{A}(B)\right) U^{-1}$. In particular, for $B:=\Phi_{A}^{-1}(f \otimes \hat{f})$ we observe $\Phi_{J}\left(U B U^{-1}\right)=U\left(\Phi_{A}(B)\right) U^{-1}=U(f \otimes$ $\hat{f}) U^{-1}=\left(U^{-1}\right)^{t} f \otimes U \hat{f}$. Applying $\Phi_{J}^{-1}$ to both sides of the equation, we get $U B U^{-1}=\Phi_{J}^{-1}\left(\left(U^{-1}\right)^{t} f \otimes U \hat{f}\right)$. Therefore we have expressed $B$ in terms of $J, U$ by $B=\Phi_{A}^{-1}(f \otimes \hat{f})=U^{-1}\left[\Phi_{J}^{-1}\left(\left(U^{-1}\right)^{t} f \otimes U \hat{f}\right)\right] U$. Hence, the following relation between $q_{ \pm}(x, t)$ and $p_{ \pm}(x, t)$ as defined in Theorem A holds,

$$
\begin{aligned}
& p_{ \pm}(x, t)= \\
& =\operatorname{det}\left(1 \pm i \exp \left(A x+A^{-1} t\right) \Phi_{A}^{-1}(f \otimes \hat{f})\right) \\
& =\operatorname{det}\left(1 \pm i \exp \left(U^{-1}\left(J x+J^{-1} t\right) U\right) \cdot U^{-1}\left[\Phi_{J}^{-1}\left(\left(U^{-1}\right)^{t} f \otimes U \hat{f}\right)\right] U\right) \\
& =\operatorname{det}\left(1 \pm i \exp \left(J x+J^{-1} t\right) \Phi_{J}^{-1}\left(\left(U^{-1}\right)^{t} f \otimes U \hat{f}\right)\right) \\
& =q_{ \pm}(x, t),
\end{aligned}
$$

yielding the assertion.

To avoid repetitions, we impose the following assumption for the rest of the paper:

Assumption 4.1. Let the matrix $A \in \mathcal{M}_{d, d}(\mathbf{C})$ in Theorem $A$ be given 
by

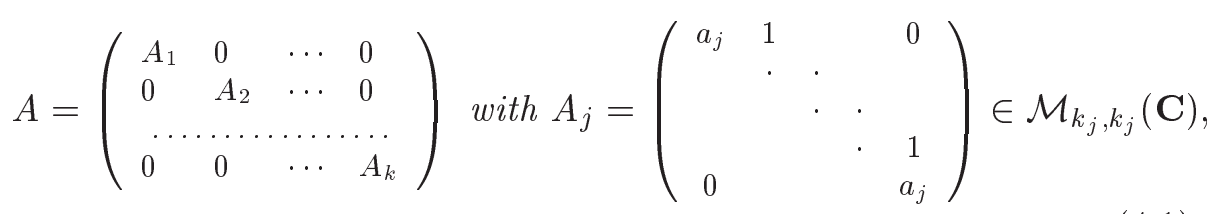

Jordan blocks of dimension $k_{j}$ corresponding to the eigenvalue $a_{j}$, in particular $d=\sum_{j=1}^{k} k_{j}$.

We assume

$$
\left|a_{i}\right| \neq\left|a_{j}\right| \quad \forall i, j \quad \text { and } \quad \Re\left(a_{j}\right)>0 \quad \forall j,
$$

and, in view of Theorem $C$, we admit as constellations of eigenvalues either real eigenvalues $a_{j} \in \mathbf{R}$ or pairs $a_{j}, \overline{a_{j}}$ of complex conjugate eigenvalues.

Remark. It should be stressed that for the reduction steps in this section we only need the assumption concerning the invertibility of $\Phi_{A}$, i.e.

$$
a_{i}+a_{j} \neq 0 \quad \forall i, j
$$

Our next step is the calculation of $\Phi_{A}^{-1}(f \otimes \hat{f})$. It is convenient to introduce a slightly extended version of this operator: For given matrices $A_{i} \in \mathcal{M}_{k_{i}, k_{i}}(\mathbf{C}), A_{j} \in \mathcal{M}_{k_{j}, k_{j}}(\mathbf{C})$, we define

$$
\Phi_{A_{i}, A_{j}}: \mathcal{M}_{k_{i}, k_{j}}(\mathbf{C}) \rightarrow \mathcal{M}_{k_{i}, k_{j}}(\mathbf{C}) \quad \text { by } \quad \Phi_{A_{i}, A_{j}}(X)=A_{i} X+X A_{j} .
$$

In the sequel we use the notation $\widehat{\partial}_{i}, \partial_{j}$ for

$$
\widehat{\partial}_{i}=\left(\frac{1}{\left(k_{i}-1\right) !} \frac{\partial^{k_{i}-1}}{\partial y_{i}^{k_{i}-1}}, \ldots, \frac{\partial}{\partial y_{i}}, 1\right)^{t}, \quad \partial_{j}=\left(1, \frac{\partial}{\partial y_{j}}, \ldots, \frac{1}{\left(k_{j}-1\right) !} \frac{\partial^{k_{j}-1}}{\partial y_{j}^{k_{j}-1}}\right)^{t} .
$$

Proposition 4.2. Let $\hat{f}_{i} \in \mathbf{C}^{k_{i}}, f_{j} \in \mathbf{C}^{k_{j}}$. For two given Jordan blocks $A_{i} \in \mathcal{M}_{k_{i}, k_{i}}(\mathbf{C}), A_{j} \in \mathcal{M}_{k_{j}, k_{j}}(\mathbf{C})$ corresponding to eigenvalues $a_{i}, a_{j}$, respectively,

$$
\Phi_{A_{i}, A_{j}}^{-1}\left(f_{j} \otimes \hat{f}_{i}\right)=\left.\left(\partial_{j} \otimes \widehat{\partial}_{i}\right) F\left(y_{i}, y_{j}\right)\right|_{\substack{y_{i}=a_{i} \\ y_{j}=a_{j}}},
$$


where $F\left(y_{i}, y_{j}\right)=\frac{F_{j}\left(y_{j}\right) \hat{F}_{i}\left(y_{i}\right)}{y_{i}+y_{j}}$ and the functions $\hat{F}_{i}, F_{j}$ are chosen appropriately such that the following relations hold:

$$
\begin{aligned}
\left.\widehat{\partial}_{i} \hat{F}_{i}\left(y_{i}\right)\right|_{y_{i}=a_{i}} & =\hat{f}_{i}, \quad \text { i. e. } \\
\left.\frac{1}{\left(k_{i}-\kappa\right) !} \frac{\partial^{k_{i}-\kappa}}{\partial y_{i}^{k_{i}-\kappa}} \hat{F}_{i}\left(y_{i}\right)\right|_{y_{i}=a_{i}} & =\hat{f}_{i}^{(\kappa)} \quad \text { for } \kappa=1, \ldots, k_{i}, \\
\left.\partial_{j} F_{j}\left(y_{j}\right)\right|_{y_{j}=a_{j}} & =f_{j}, \quad \text { i. e. } \\
\left.\frac{1}{(\kappa-1) !} \frac{\partial^{\kappa-1}}{\partial y_{j}^{\kappa-1}} F_{j}\left(y_{j}\right)\right|_{y_{j}=a_{j}} & =f_{j}^{(\kappa)} \quad \text { for } \kappa=1, \ldots, k_{j} .
\end{aligned}
$$

Proof. Let $Y_{i} \in \mathcal{M}_{k_{i}, k_{i}}(\mathbf{C}), Y_{j} \in \mathcal{M}_{k_{j}, k_{j}}(\mathbf{C})$ be Jordan blocks corresponding to eigenvalues which coincide with the variables $y_{i}, y_{j}$. We easily calculate

$$
\begin{aligned}
Y_{i} \widehat{\partial}_{i} & =\left(y_{i} \frac{1}{\left(k_{i}-1\right) !} \frac{\partial^{k_{i}-1}}{\partial y_{i}^{k_{i}-1}}+\frac{1}{\left(k_{i}-2\right) !} \frac{\partial^{k_{i}-2}}{\partial y_{i}^{k_{i}-2}}, \ldots, y_{i} \frac{\partial}{\partial y_{i}}+1, y_{i}\right)^{t} \\
& =\left(\frac{1}{\left(k_{i}-1\right) !} \frac{\partial^{k_{i}-1}}{\partial y_{i}^{k_{i}-1}} y_{i}, \ldots, \frac{\partial}{\partial y_{i}} y_{i}, y_{i}\right)^{t} \\
& =\widehat{\partial}_{i} y_{i},
\end{aligned}
$$

and analogously

$$
\begin{aligned}
Y_{j}^{t} \partial_{j} & =\left(y_{j}, 1+y_{j} \frac{\partial}{\partial y_{j}}, \ldots, \frac{1}{\left(k_{j}-2\right) !} \frac{\partial^{k_{j}-2}}{\partial y_{j}^{k_{j}-2}}+y_{j} \frac{1}{\left(k_{j}-1\right) !} \frac{\partial^{k_{j}-1}}{\partial y_{j}^{k_{j}-1}}\right)^{t} \\
& =\left(y_{j}, \frac{\partial}{\partial y_{j}} y_{j}, \ldots, \frac{1}{\left(k_{j}-1\right) !} \frac{\partial^{k_{j}-1}}{\partial y_{j}^{k_{j}-1}} y_{j}\right)^{t} \\
& =\partial_{j} y_{j} .
\end{aligned}
$$

Now it is straightforward to verify that the operator $X$ defined by the right hand side of (4.4) satisfies $A_{i} X+X A_{j}=f_{j} \otimes \hat{f}_{i}$ :

$\left.A_{i}\left(\left(\partial_{j} \otimes \widehat{\partial}_{i}\right) F\left(y_{i}, y_{j}\right)\right)\right|_{\substack{y_{i}=a_{i} \\ y_{j}=a_{j}}}+\left.\left(\left(\partial_{j} \otimes \widehat{\partial}_{i}\right) F\left(y_{i}, y_{j}\right)\right)\right|_{\substack{y_{i}=a_{i} \\ y_{j}=a_{j}}} A_{j}$
$=\left.\left(Y_{i}\left(\left(\partial_{j} \otimes \widehat{\partial}_{i}\right) F\left(y_{i}, y_{j}\right)\right)+\left(\left(\partial_{j} \otimes \widehat{\partial}_{i}\right) F\left(y_{i}, y_{j}\right)\right) Y_{j}\right)\right|_{\substack{y_{i}=a_{i} \\ y_{j}=a_{j}}}$ 


$$
\begin{aligned}
& =\left.\left(\left(\partial_{j} \otimes\left(Y_{i} \widehat{\partial}_{i}\right) F\left(y_{i}, y_{j}\right)\right)+\left(\left(Y_{j}^{t} \partial_{j}\right) \otimes \widehat{\partial}_{i} F\left(y_{i}, y_{j}\right)\right)\right)\right|_{\substack{y_{i}=a_{i} \\
y_{j}=a_{j}}} \\
& =\left.\left(\left(\partial_{j} \otimes \widehat{\partial}_{i}\right)\left(\left(y_{i}+y_{j}\right) F\left(y_{i}, y_{j}\right)\right)\right)\right|_{\substack{y_{i}=a_{i} \\
y_{j}=a_{j}}} \\
& =\left.\left.\left(\partial_{j} F_{j}\left(y_{j}\right)\right)\right|_{y_{j}=a_{j}} \otimes\left(\widehat{\partial}_{i} \hat{F}_{i}\left(y_{i}\right)\right)\right|_{y_{i}=a_{i}} \\
& =f_{j} \otimes \hat{f}_{i} \text {. }
\end{aligned}
$$

Recall the notion of Toeplitz matrices for matrices $T$ of the form $T=\left(t_{i-j}\right)_{i, j}$.

Corollary 4.3. If $\widehat{T}_{i} \in \mathcal{M}_{k_{i}, k_{i}}(\mathbf{C}), T_{j} \in \mathcal{M}_{k_{j}, k_{j}}(\mathbf{C})$ are upper triangular Toeplitz matrices, uniquely determined by $\widehat{T}_{i}(0, \ldots, 0,1)^{t}=\hat{f}_{i}$ and $T_{j}^{t}(1,0, \ldots, 0)^{t}=f_{j}$, the following factorization holds:

$$
\Phi_{A_{i}, A_{j}}^{-1}\left(f_{j} \otimes \hat{f}_{i}\right)=\widehat{T}_{i}\left(\left.\left(\partial_{j} \otimes \widehat{\partial}_{i}\right) \frac{1}{y_{i}+y_{j}}\right|_{\substack{y_{i}=a_{i} \\ y_{j}=a_{j}}}\right) T_{j} .
$$

Proof. Choosing $\hat{F}_{i}, F_{j} \equiv 1$, Proposition ?? yields

$$
\begin{gathered}
A_{i}\left(\left.\left(\partial_{j} \otimes \widehat{\partial}_{i}\right) \frac{1}{y_{i}+y_{j}}\right|_{\substack{y_{i}=a_{i} \\
y_{j}=a_{j}}}\right)+\left(\left.\left(\partial_{j} \otimes \widehat{\partial}_{i}\right) \frac{1}{y_{i}+y_{j}}\right|_{\substack{y_{i}=a_{i} \\
y_{j}=a_{j}}}\right) A_{j}= \\
=(1,0, \ldots, 0)^{t} \otimes(0, \ldots, 0,1)^{t} .
\end{gathered}
$$

Since upper triangular Toeplitz matrices obviously commute,

$$
\begin{aligned}
& A_{i}\left[\widehat{T}_{i}\left(\left.\left(\partial_{j} \otimes \widehat{\partial}_{i}\right) \frac{1}{y_{i}+y_{j}}\right|_{\substack{y_{i}=a_{i} \\
y_{j}=a_{j}}}\right) T_{j}\right]+\left[\widehat{T_{i}}\left(\left.\left(\partial_{j} \otimes \widehat{\partial}_{i}\right) \frac{1}{y_{i}+y_{j}}\right|_{\substack{y_{i}=a_{i} \\
y_{j}=a_{j}}}\right) T_{j}\right] A_{j} \\
= & \widehat{T}_{i}\left[A_{i}\left(\left.\left(\partial_{j} \otimes \widehat{\partial}_{i}\right) \frac{1}{y_{i}+y_{j}}\right|_{\substack{y_{i}=a_{i} \\
y_{j}=a_{j}}}\right)+\left(\left.\left(\partial_{j} \otimes \widehat{\partial}_{i}\right) \frac{1}{y_{i}+y_{j}}\right|_{\substack{y_{i}=a_{i} \\
y_{j}=a_{j}}}\right) A_{j}\right] T_{j} \\
= & \widehat{T}_{i}\left((1,0, \ldots, 0)^{t} \otimes(0, \ldots, 0,1)^{t}\right) T_{j} \\
= & \left(T_{j}^{t}(1,0, \ldots, 0)^{t}\right) \otimes\left(\widehat{T}_{i}(0, \ldots, 0,1)^{t}\right) \\
= & f_{j} \otimes \hat{f}_{i},
\end{aligned}
$$


and the assumption follows by the uniqueness of $\Phi_{A_{i}, A_{j}}^{-1}\left(f_{j} \otimes \hat{f}_{i}\right)$.

Now we are prepared for the announced reduction.

Proposition 4.4. With the notations of this section, decomposing the vectors $f, \hat{f} \in \mathbf{C}^{d}$ accordingly $f=\left(f_{1}, \ldots, f_{k}\right)^{t}$, where $f_{j}=\left(f_{j}^{(1)}, \ldots, f_{j}^{\left(k_{j}\right)}\right)^{t}$ for $j=1, \ldots, k$, we observe the following reformulation of the determinants $p_{ \pm}(x, t)$ in Theorem $A$ :

$$
\begin{aligned}
p_{ \pm}(x, t) & = \\
& =\operatorname{det}\left(1 \pm \mathrm{i}\left(\left.L_{i}\right|_{y_{i}=a_{i}}\left(\left.\left(\partial_{j} \otimes \widehat{\partial}_{i}\right) \frac{1}{y_{i}+y_{j}}\right|_{\substack{y_{i}=a_{i} \\
y_{j}=a_{j}}}\right)\right)_{i, j=1}^{k}\right) \\
& \left.=\operatorname{det}\left(1 \pm \mathrm{i}\left(\left(\partial_{j} \otimes \partial_{i}\right) \frac{1}{y_{i}+y_{j}}\right) L_{j} D_{j}\right)_{i, j=1}^{k}\right)\left.\right|_{y_{j}=a_{j} \forall j} \\
\text { with } D_{i} & =\left(\begin{array}{cccc}
0 & \cdots & 0 & 1 \\
0 & \cdots & 1 & 0 \\
\cdots & \cdots & \cdots \\
1 & \cdots & 0 & 0
\end{array}\right) \in \mathcal{M}_{k_{i}, k_{i}}(\mathbf{C}) \\
\text { and } L_{i} & =\left(\begin{array}{cccc}
\ell_{i}^{(0)} & \ell_{i}^{(1)} & \\
& \cdot & \cdot \\
\ell_{i}^{\left(k_{i}-1\right)} \\
0 & \cdot & \cdot \\
0 & \cdot & \ell_{i}^{(1)} \\
0 & & \ell_{i}^{(0)}
\end{array}\right) \in \mathcal{M}_{k_{i}, k_{i}}(\mathbf{C})
\end{aligned}
$$

an upper triangular Toeplitz matrix with entries $\ell_{i}^{(\kappa)}\left(y_{i} ; x, t\right)=\frac{1}{\kappa !} \frac{\partial^{\kappa}}{\partial y_{i}^{\kappa}} \ell_{i}\left(y_{i} ; x, t\right), \kappa=0, \ldots, k_{i}-1$, where the underlying function is $\ell_{i}\left(y_{i} ; x, t\right)=\exp \left(y_{i} x+y_{i}^{-1} t+f_{i}\left(y_{i}\right)\right)$ with $\exp \left(f_{i}\left(y_{i}\right)\right)=$ $F_{i}\left(y_{i}\right) \hat{F}_{i}\left(y_{i}\right)$ and the coefficients $F_{i}\left(y_{i}\right), \hat{F}_{i}\left(y_{i}\right)$ are defined by (4.5), (4.6).

Proof. Since $D_{i} \partial_{i}=\widehat{\partial}_{i}$, it is sufficient to prove (4.7).

Concerning the specified decomposition, the determinant reads

$$
\begin{aligned}
p_{ \pm}(x, t) & =\operatorname{det}\left(1 \pm \mathrm{i} \exp \left(A x+A^{-1} t\right) \Phi_{A}^{-1}(f \otimes \hat{f})\right) \\
& =\operatorname{det}\left(1 \pm \mathrm{i}\left(\exp \left(A_{i} x+A_{i}^{-1} t\right) \Phi_{A_{i}, A_{j}}^{-1}\left(f_{j} \otimes \hat{f}_{i}\right)\right)_{i, j=1}^{k}\right) .
\end{aligned}
$$


By Corollary 4.2, $\Phi_{A_{i}, A_{j}}^{-1}\left(f_{j} \otimes \hat{f}_{i}\right)=\widehat{T}_{i}\left(\left.\left(\partial_{j} \otimes \widehat{\partial}_{i}\right) \frac{1}{y_{i}+y_{j}}\right|_{\substack{y_{i}=a_{i} \\ y_{j}=a_{j}}}\right) T_{j}$, where $\widehat{T}_{i}, T_{j}$ are upper triangular Toeplitz matrices determined by $\widehat{T}_{i}(0, \ldots, 0,1)^{t}=\hat{f}_{i} \forall i$ and $T_{j}^{t}(1,0, \ldots, 0)^{t}=f_{j} \forall j$. If we define functions $F_{j}\left(y_{j}\right), \hat{F}_{i}\left(y_{i}\right)$ by $(4.5),(4.6)$, this is equivalent to

$$
\begin{aligned}
\widehat{T}_{i}(0, \ldots, 0,1)^{t} & =\left.\widehat{\partial}_{i} \hat{F}_{i}\left(y_{i}\right)\right|_{y_{i}=a_{i}}, \\
T_{j}^{t}(1,0, \ldots, 0)^{t} & =\left.\partial_{j} F_{j}\left(y_{j}\right)\right|_{y_{j}=a_{j}} \Leftrightarrow T_{j}(0, \ldots, 0,1)^{t}=\left.\widehat{\partial}_{j} F_{j}\left(y_{j}\right)\right|_{y_{j}=a_{j}} .
\end{aligned}
$$

From the properties of a determinant, we infer $p_{ \pm}(x, t)=$

$$
=\operatorname{det}\left(1 \pm \mathrm{i}\left(\left(T_{i} \exp \left(A_{i} x+A_{i}^{-1} t\right) \widehat{T}_{i}\right)\left(\left.\left(\partial_{j} \otimes \widehat{\partial}_{i}\right) \frac{1}{y_{i}+y_{j}}\right|_{\substack{y_{i}=a_{i} \\ y_{j}=a_{j}}}\right)\right)_{i, j=1}^{k}\right) \text {. }
$$

A straightforward calculation shows that $\exp \left(A_{i} x+A_{i}^{-1} t\right)$ is an upper triangular Toeplitz matrix as well. It is determined by

$$
\begin{gathered}
\exp \left(A_{i} x+A_{i}^{-1} t\right)(0, \ldots, 0,1)^{t}= \\
=\left(\frac{1}{\left(k_{i}-1\right) !} \frac{\partial^{k_{i}-1}}{\partial a_{i}^{k_{i}-1}} e^{a_{i} x+a_{i}^{-1} t}, \ldots, e^{a_{i} x+a_{i}^{-1} t}\right)^{t}=\left.\widehat{\partial}_{i} e^{y_{i} x+y_{i}^{-1} t}\right|_{y_{i}=a_{i}} .
\end{gathered}
$$

Therefore, we finally observe

$$
\begin{aligned}
& \left(T_{i} \exp \left(A_{i} x+A_{i}^{-1} t\right) \widehat{T}_{i}\right)(0, \ldots, 0,1)^{t} \\
& =\left(T_{i} \exp \left(A_{i} x+A_{i}^{-1} t\right)\right)\left(\left.\frac{1}{\left(k_{i}-\kappa\right) !} \frac{\partial^{k_{i}-\kappa}}{\partial y_{i}^{k_{i}-\kappa}} \hat{F}_{i}\left(y_{i}\right)\right|_{y_{i}=a_{i}}\right)_{\kappa=1}^{k} \\
& =T_{i}\left(\left.\left.\sum_{\kappa^{\prime}=\kappa}^{k} \frac{1}{\left(\kappa^{\prime}-\kappa\right) !} \frac{\partial^{\kappa^{\prime}-\kappa}}{\partial y_{i}^{\kappa^{\prime}-\kappa}} e^{y_{i} x+y_{i}^{-1} t}\right|_{y_{i}=a_{i}} \frac{1}{\left(k_{i}-\kappa^{\prime}\right) !} \frac{\partial^{k_{i}-\kappa^{\prime}}}{\partial y_{i}^{k_{i}-\kappa^{\prime}}} \hat{F}_{i}\left(y_{i}\right)\right|_{y_{i}=a_{i}}\right)_{\kappa=1}^{k} \\
& =T_{i}\left(\left.\frac{1}{\left(k_{i}-\kappa\right) !} \frac{\partial^{k_{i}-\kappa}}{\partial y_{i}^{k_{i}-\kappa}}\left(\hat{F}_{i}\left(y_{i}\right) e^{y_{i} x+y_{i}^{-1} t}\right)\right|_{y_{i}=a_{i}}\right)_{\kappa=1}^{k} \\
& =\left(\left.\left.\sum_{\kappa^{\prime}=\kappa}^{k} \frac{1}{\left(\kappa^{\prime}-\kappa\right) !} \frac{\partial^{\kappa^{\prime}-\kappa}}{\partial y_{i}^{k^{\prime}-\kappa}} F_{i}\left(y_{i}\right)\right|_{y_{i}=a_{i}} \frac{1}{\left(k_{i}-\kappa^{\prime}\right) !} \frac{\partial^{k_{i}-\kappa^{\prime}}}{\partial y_{i}^{k_{i}-\kappa^{\prime}}} \hat{F}_{i}\left(y_{i}\right) e^{y_{i} x+y_{i}^{-1} t}\right|_{y_{i}=a_{i}}\right)_{\kappa=1}^{k} \\
& =\left(\left.\frac{1}{\left(k_{i}-\kappa\right) !} \frac{\partial^{k_{i}-\kappa}}{\partial y_{i}^{k_{i}-\kappa}}\left(F_{i}\left(y_{i}\right) \hat{F}_{i}\left(y_{i}\right) e^{y_{i} x+y_{i}^{-1} t}\right)\right|_{y_{i}=a_{i}}\right)_{\kappa=1}^{k} \\
& =L_{i}(0, \ldots, 0,1)^{t} .
\end{aligned}
$$




\section{$5 \quad$ Regularity of clusters}

The main purpose of this section is to show that the solutions we deal with are well-defined, that is $p_{ \pm}(x, t) \neq 0 \forall(x, t)$. Although this aspect is often neglected in the literature, it is sometimes far away from being obvious.

Recall that two operators $T \in \mathcal{L}(E), \hat{T} \in \mathcal{L}(\hat{E})$ are called related if there exist $A \in \mathcal{L}(\hat{E}, E), B \in \mathcal{L}(E, \hat{E})$ such that $T=A B$ and $\hat{T}=B A$. It is clear from the definition that $\operatorname{det}(1+T)=\operatorname{det}(1+\hat{T})$ for related operators (if the determinant exists).

Proposition 5.1. Assume $\Re\left(a_{j}\right)>0 \forall j$. Then the operator $L(x, t)=$ $\exp \left(A x+A^{-1} t\right) \Phi_{A}^{-1}(f \otimes \hat{f})$ on $\bigoplus_{j=1}^{k} \ell_{2}^{k_{j}}$ is related to the integral operator $H$ on $L_{2}(-\infty, 0]$ given by

$$
\begin{gathered}
(H f)(s)=\int_{-\infty}^{0} f(\sigma) h(s, \sigma) d \sigma \quad \text { with kernel } \\
h(s, \sigma)=\left.\sum_{j=1}^{k} \frac{1}{\left(k_{j}-1\right) !} \frac{\partial^{k_{j}-1}}{\partial y_{j}^{k_{j}-1}}\left(\ell_{j}^{(0)}\left(y_{j} ; x, t\right) \exp \left(y_{j}(s+\sigma)\right)\right)\right|_{y_{j}=a_{j}},
\end{gathered}
$$

where we used the notation $\ell_{j}^{(0)}\left(y_{j} ; x, t\right)=\exp \left(y_{j} x+y_{j}^{-1} t+f_{j}\left(y_{j}\right)\right)($ confer Proposition 4.4).

If, in addition, the $a_{j}$ form a symmetric set $\mathcal{A}$ (that is $a_{j} \in \mathcal{A} \Longrightarrow$ $\left.\bar{a}_{j} \in \mathcal{A}\right)$, and, moreover,

$$
\left.\frac{\partial^{\kappa}}{\partial y_{j}^{\kappa}} \exp \left(f_{j}\left(y_{j}\right)\right)\right|_{y_{j}=\overline{a_{j}}}=\overline{\left.\frac{\partial^{\kappa}}{\partial y_{j}^{\kappa}} \exp \left(f_{j}\left(y_{j}\right)\right)\right|_{y_{j}=a_{j}}},
$$

then $H$ is hermitian and thus has only real eigenvalues.

In particular, in this situation, $\operatorname{det}(1-\mathrm{i} L(x, t)) \neq 0$.

Proof. By $\left\{e_{j}^{\left(j^{\prime}\right)} \mid j^{\prime}=1, \ldots, k_{j}\right\}$ we denote the canonical basis in $\ell_{2}^{k_{j}}$ $(j=1, \ldots, k)$. 
Abbreviating $\ell_{j}\left(y_{j}\right)=\ell_{j}^{(0)}\left(y_{j} ; x, t\right)$, we define

$$
\begin{gathered}
T: \bigoplus_{j=1}^{k} \ell_{2}^{k_{j}} \longrightarrow L_{2}[-\infty, 0) \quad \text { by } \\
\left(T e_{j}^{\left(j^{\prime}\right)}\right)(\sigma)=\left.\frac{1}{\left(j^{\prime}-1\right) !} \frac{\partial^{j^{\prime}-1}}{\partial y_{j}^{j^{\prime}-1}}\left(\exp \left(y_{j} \sigma\right)\right)\right|_{y_{j}=a_{j}}, \\
S: L_{2}[-\infty, 0) \longrightarrow \bigoplus_{j=1}^{k} \ell_{2}^{k_{j}} \quad \text { by } \\
S f=\sum_{j=1}^{k} \sum_{j^{\prime}=1}^{k_{j}}\left(\left.\int_{-\infty}^{0} f(s) \frac{1}{\left(k_{j}-j^{\prime}\right) !} \frac{\partial^{k_{j}-j^{\prime}}}{\partial y_{j}^{k_{j}-j^{\prime}}}\left(\ell_{j}\left(y_{j}\right) \exp \left(y_{j} s\right)\right)\right|_{y_{j}=a_{j}} d s\right) e_{j}^{\left(j^{\prime}\right)} .
\end{gathered}
$$

First observe

$$
\begin{aligned}
& \left\langle S T e_{j}^{\left(j^{\prime}\right)}, e_{i}^{\left(i^{\prime}\right)}\right\rangle= \\
& =\left.\left.\int_{-\infty}^{0} \frac{1}{\left(j^{\prime}-1\right) !} \frac{\partial^{j^{\prime}-1}}{\partial y_{j}^{j^{\prime}}-1}\left(\exp \left(y_{j} s\right)\right)\right|_{y_{j}=a_{j}} \cdot \frac{1}{\left(k_{i}-i^{\prime}\right) !} \frac{\partial^{k_{i}-i^{\prime}}}{\partial y_{i}^{k_{i}-i^{\prime}}}\left(\ell_{i}\left(y_{i}\right) \exp \left(y_{i} s\right)\right)\right|_{y_{i}=a_{i}} d s \\
& =\left.\sum_{i^{\prime \prime}=0}^{k_{i}-i^{\prime}} \frac{1}{\left(k_{i}-i^{\prime}-i^{\prime \prime}\right) !} \frac{\partial^{k_{i}-i^{\prime}-i^{\prime \prime}}}{\partial y_{i}^{k_{i}-i^{\prime}-i^{\prime \prime}}} \ell_{i}\left(y_{i}\right) \int_{-\infty}^{0} \frac{1}{\left(j^{\prime}-1\right) !} \frac{\partial^{j^{\prime}-1}}{\partial y_{j}^{j^{\prime}}-1} \frac{1}{i^{\prime \prime} !} \frac{\partial^{i^{\prime \prime}}}{\partial y_{i}^{i^{\prime \prime}}} \exp \left(\left(y_{i}+y_{j}\right) s\right) d s\right|_{\substack{y_{i}=a_{i} \\
y_{j}=a_{j}}}
\end{aligned}
$$

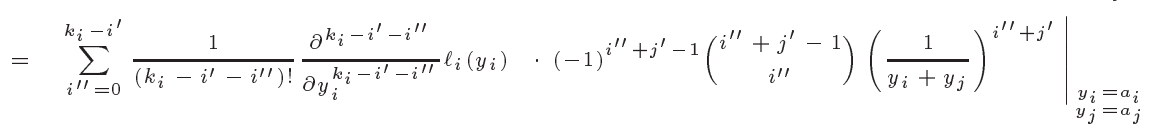

$$
\begin{aligned}
& =\left.\frac{1}{\left(j^{\prime}-1\right) !} \frac{\partial^{j^{\prime}-1}}{\partial y_{j}^{j^{\prime}-1}} \frac{1}{\left(k_{i}-i^{\prime}\right) !} \frac{\partial^{k_{i}-i^{\prime}}}{\partial y_{i}^{k_{i}-i^{\prime}}} \frac{\ell_{i}\left(y_{i}\right)}{y_{i}+y_{j}}\right|_{\substack{y_{i}=a_{i} \\
y_{j}=a_{j}}} .
\end{aligned}
$$

On the other hand, by the reduction proved in Proposition 4.4,

$$
\begin{aligned}
& L(x, t)=\left.\left(L_{i}\left(\partial_{j} \otimes \widehat{\partial}_{i}\right) \frac{1}{y_{i}+y_{j}}\right)_{i, j=1}^{k}\right|_{\substack{y_{i}=a_{i} \\
y_{j}=a_{j}}} \\
& =\left.\left(\left(\partial_{j} \otimes \widehat{\partial}_{i}\right) \frac{\ell_{i}^{(0)}}{y_{i}+y_{j}}\right)^{k}\right|_{\substack{i, j=1 \\
y_{i}=a_{i} \\
y_{j}=a_{j}}},
\end{aligned}
$$


and it is easy to see that this expression coincides with $\left(\left(\left\langle S T e_{j}^{\left(j^{\prime}\right)}, e_{i}^{\left(i^{\prime}\right)}\right\rangle\right)_{\substack{i^{\prime}=1, \ldots, k_{i} \\ j^{\prime}=1, \ldots, k_{j}}}\right)_{i, j=1}^{k}$. Thus we obtained a factorization of $L(x, t)=S T$ through the Hilbert space $L_{2}(-\infty, 0]$.

Vice versa, $(T S f)(\sigma)=$

$=\left.\sum_{j=1}^{k} \sum_{j^{\prime}=1}^{k_{j}} \int_{-\infty}^{0} f(s)\left(\frac{1}{\left(k_{j}-j^{\prime}\right) !} \frac{\partial^{k_{j}-j^{\prime}}}{\partial y_{j}^{k_{j}-j^{\prime}}}\left(\ell_{j}\left(y_{j}\right) \exp \left(y_{j} s\right)\right)\right) d s \cdot \frac{1}{\left(j^{\prime}-1\right) !} \frac{\partial^{j^{\prime}-1}}{\partial y_{j}^{j^{\prime}-1}}\left(\exp \left(y_{j} \sigma\right)\right)\right|_{y_{j}=a_{j}}$ $=\left.\int_{-\infty}^{0} f(s)\left(\sum_{j=1}^{k} \frac{1}{\left(k_{j}-1\right) !} \sum_{j^{\prime}=1}^{k_{j}}\left(\begin{array}{c}k_{j}-1 \\ j^{\prime}-1\end{array}\right) \frac{\partial^{k_{j}-j^{\prime}}}{\partial y_{j}^{k_{j}-j^{\prime}}}\left(\ell_{j}\left(y_{j}\right) \exp \left(y_{j} s\right)\right) \frac{\partial^{j^{\prime}-1}}{\partial y_{j}^{j^{\prime}-1}}\left(\exp \left(y_{j} \sigma\right)\right)\right)\right|_{y_{j}=a_{j}} d s$ $=\left.\int_{-\infty}^{0} f(s)\left(\sum_{j=1}^{k} \frac{1}{\left(k_{j}-1\right) !} \frac{\partial^{k_{j}-1}}{\partial y_{j} k^{-1}}\left(\ell_{j}\left(y_{j}\right) \exp \left(y_{j}(s+\sigma)\right)\right)\right)\right|_{y_{j}=a_{j}} d s$ $=(H f)(\sigma)$.

Under the additional assumptions, $h(s, \sigma)$ is real-valued, and, since $h(s, \sigma)=h(\sigma, s)$ anyway, it represents a hermitian operator.

Remark. By the way, Proposition 5.1 establishes a nice relation to the methods of Pöppe [19]. In fact he uses the Fredholm integral operator given by the kernel $h(s, \sigma)$ to construct multipole solutions.

\section{Asymptotics of clusters}

This section contains the asymptotic analysis.

First of all, let us slightly reformulate the solution formula of Section 4.

According to Assumption 4.1 and the assumption on $f$ and $\hat{f}$ in Theorem C, which guarantees that $\left.\frac{\partial^{\kappa}}{\partial y_{j}^{\kappa}} \exp \left(f_{j}\left(y_{j}\right)\right)\right|_{y_{j}=a_{j}}$ is real-valued for real

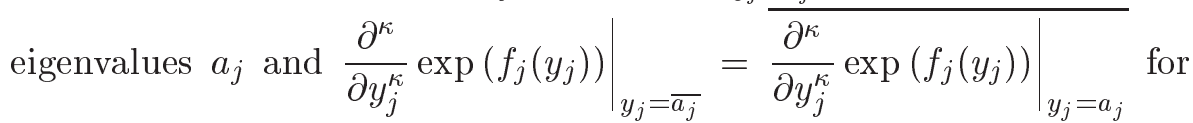
pairs of complex conjugated eigenvalues $a_{j}, \overline{a_{j}}$, it is not difficult to check that the determinants $p_{ \pm}(x, t)$ in Theorem $\mathrm{A}$ are complex conjugate to 
each other. Thus, with $p(x, t):=p_{-}(x, t)$, our solution formula reads

$u=2 \mathrm{i} \log \frac{p}{\bar{p}} \quad$ with $p=\operatorname{det}\left(\delta_{j j^{\prime}} \mathbf{1}_{k_{j} k_{j^{\prime}}}-\mathrm{i} W_{j j^{\prime}}\left(L_{j^{\prime}} D_{j^{\prime}}\right)\right)_{j, j^{\prime}=1}^{k}$,

where $\quad W_{j j^{\prime}}=\left((-1)^{\kappa+\kappa^{\prime}-1}\left(\frac{1}{a_{j}+a_{j^{\prime}}}\right)^{\kappa+\kappa^{\prime}-1}\left(\begin{array}{c}(\kappa-1)+\left(\kappa^{\prime}-1\right) \\ (\kappa-1)\end{array}\right)\right)_{\substack{\kappa=1, \ldots, k_{j} \\ \kappa^{\prime}=1, \ldots, k_{j^{\prime}}}}(6$

As we will see, the term $\operatorname{det}\left(W_{j j^{\prime}}\right)_{j j^{\prime}}$ is responsible for the phaseshifts. Therefore, the value of $\operatorname{det}\left(W_{j j^{\prime}}\right)_{j j^{\prime}}$ is a crucial point in the asymptotic analysis. It is given by the following theorem.

Theorem 6.1. For the determinant of the matrix $\hat{W}=\left(\hat{W}_{j j^{\prime}}\right)_{j, j^{\prime}=1}^{k}$ consisting of the blocks $\hat{W}_{j j^{\prime}}=\left(\left(\frac{1}{a_{j}+a_{j^{\prime}}}\right)^{\kappa+\kappa^{\prime}-1}\left(\begin{array}{c}(\kappa-1)+\left(\kappa^{\prime}-1\right) \\ (\kappa-1)\end{array}\right)\right)_{\substack{\kappa=1, \ldots, k_{j} \\ \kappa^{\prime}=1, \ldots, k_{j^{\prime}}}}$, where $a_{j}+a_{j^{\prime}} \neq 0$,

$$
\operatorname{det}(\hat{W})=\prod_{j=1}^{k}\left[\frac{1}{\left(a_{j}+a_{j^{\prime}}\right)}\right]^{k_{j}^{2}} \cdot \prod_{\substack{j, j^{\prime}=1 \\ j<j^{\prime}}}^{k}\left[\frac{\left(a_{j}-a_{j^{\prime}}\right)}{\left(a_{j}+a_{j^{\prime}}\right)}\right]^{2 k_{j} k_{j^{\prime}}} .
$$

The proof of this theorem, which indeed is very involved, is beyond the scope ot the present article. We thus omit it and point the interested reader to the author's thesis [22] instead.

Remark. Theorem 6.1 is a generalization of the well-known formula for Jacobi's determinant $\operatorname{det}\left(\frac{1}{a_{j}+a_{j^{\prime}}}\right)_{j j^{\prime}}$ (see Pólya and Szegö [20]), which is included in Theorem 6.1 as the special case $k_{j}=1 \forall j$.

There is another lemma which will be needed throughout the asymptotic analysis. Since its proof is straightforward, we omit it, too.

Lemma 6.2. Let $A=\left(a_{i j}\right)_{i, j=1}^{N}, B=\left(b_{i j}\right)_{i, j=1}^{N}$ be matrices the entries of which grow according the estimates $\left|a_{i j}\right| \leq p_{a}(\tau) \exp (\alpha \tau)$ and $\left|b_{i j}\right| \leq$ $p_{b}(\tau) \exp (-\beta \tau) \forall \tau \geq \tau_{0}$, where $p_{a}, p_{b}$ are polynomials and $\alpha, \beta>0$. If $\gamma:=\beta-N \alpha>0$, then there is a polynomial $P$ such that

$$
|\operatorname{det}(A+B)-\operatorname{det}(A)| \leq P(\tau) \exp (-\gamma \tau) \quad \forall \tau \geq \tau_{0}
$$


Let us next briefly provide some reformulations which are necessary because the asymptotic description is done in the variables $\xi, \tau$. Recall that

$$
\begin{gathered}
\left.\ell_{j}^{(\kappa)}\left(y_{j} ; \xi, \tau\right)\right|_{y_{j}=a_{j}}=\left.\left.\frac{1}{\kappa !} p_{j}^{(\kappa)}\left(y_{j} ; \xi, \tau\right)\right|_{y_{j}=a_{j}} \ell_{j}\left(y_{j} ; \xi, \tau\right)\right|_{y_{j}=a_{j}} \\
\text { with } p_{j}^{(\kappa)}\left(y_{j} ; \xi, \tau\right)=\left(\frac{\partial^{\kappa}}{\partial y_{j}^{\kappa}} \ell_{j}\left(y_{j} ; \xi, \tau\right)\right) / \ell_{j}\left(y_{j} ; \xi, \tau\right) .
\end{gathered}
$$

Obviously, $p_{j}^{(\kappa)}\left(y_{j} ; \xi, \tau\right)$ are polynomials in $(\xi, \tau)$ (for all $\kappa$ ). Moreover, the recursion

$$
p_{j}^{(\kappa)}\left(y_{j} ; \xi, \tau\right)=\left(\frac{\partial}{\partial y_{j}} p_{j}^{(\kappa-1)}\left(y_{j} ; \xi, \tau\right)\right)+p_{j}^{(1)}\left(y_{j} ; \xi, \tau\right) p_{j}^{(\kappa-1)}\left(y_{j} ; \xi, \tau\right)
$$

for $\kappa \geq 1$ holds. Thus

$$
p_{j}^{(\kappa)}\left(y_{j} ; \xi, \tau\right)=\left(p_{j}^{(1)}\left(y_{j} ; \xi, \tau\right)\right)^{\kappa}+P_{j, \kappa}\left(y_{j} ; \xi, \tau\right),
$$

where $P_{j, \kappa}$ are polynomials in $\xi, \tau$ of order less than $\kappa-1$.

In the sequel we suppress the dependence on the variables $y_{j}$ as well as the evaluation at $y_{j}=a_{j}$, for example $\ell_{j}(\xi, \tau)=\left.\ell_{j}\left(y_{j} ; \xi, \tau\right)\right|_{y_{j}=a_{j}}$. In terms of the variables $\xi, \tau$ we have

$$
\begin{aligned}
\ell_{j}(\xi, \tau) & =\exp \left(\sqrt{1-\omega_{j}^{2}} \frac{\xi-\lambda_{j} \tau}{\sqrt{1-\lambda_{j}^{2}}}-\mathrm{i} \omega_{j} \frac{\tau-\lambda_{j} \xi}{\sqrt{1-\lambda_{j}^{2}}}+f_{j}\left(a_{j}\right)\right), \\
p_{j}^{(1)}(\xi, \tau) & =\frac{1}{a_{j}}\left(-\sqrt{1-\omega_{j}^{2}} \frac{\tau-\lambda_{j} \xi}{\sqrt{1-\lambda_{j}^{2}}}+\mathrm{i} \omega_{j} \frac{\xi-\lambda_{j} \tau}{\sqrt{1-\lambda_{j}^{2}}}\right)+f_{j}^{\prime}\left(a_{j}\right)
\end{aligned}
$$

with the notation $a_{j}=\left|a_{j}\right|\left(\sqrt{1-\omega_{j}^{2}}+\mathrm{i} \omega_{j}\right), \lambda_{j}=-\frac{1-\left|a_{j}\right|^{2}}{1+\left|a_{j}\right|^{2}}$.

With this preparation in mind, we carry out the asymptotic analysis in two steps: At first, we convince ourselves that all clusters are separated, and, secondly, we examine the participating waves within a single cluster.

We concentrate on $\tau \rightarrow+\infty$. The arguments for $\tau \rightarrow-\infty$ are completely parallel. 
Step 1. With the abbreviation $\Lambda_{j_{0}}^{+}=\left\{j: \lambda_{j}<\lambda_{j_{0}}\right\}, \Lambda_{j_{0}}^{-}=\left\{j: \lambda_{j_{0}}<\right.$ $\left.\lambda_{j}\right\}$, the following holds: $\{1 \ldots, k\}=\Lambda_{j_{0}}^{+} \cup \Lambda_{j_{0}}^{-} \cup\left\{j_{0}, \bar{\jmath}_{0}\right\}$, where $\bar{\jmath}_{0}=j_{0}$ for $\Im\left(a_{j_{0}}\right)=0$ and $\bar{\jmath}_{0} \neq j_{0}$ for $\Im\left(a_{j_{0}}\right) \neq 0$, that is $a_{\bar{\jmath}_{0}}=\bar{a}_{j_{0}}$. We show

$$
\begin{gathered}
u(\xi, \tau) \approx \sum_{j 0: \Im\left(a_{j_{0}}\right) \geq 0} u_{a_{j_{0}}}^{+}(\xi, \tau), \\
u_{a_{j_{0}}}^{+}=2 \mathrm{i} \log \frac{p_{a_{j_{0}}}^{+}}{p_{a_{j_{0}}^{+}}^{+}}-c_{j_{0}}^{+} \text {and } p_{a_{j_{0}}}^{+}=\operatorname{det}\left(V_{j j^{\prime}}^{\left(j_{0}\right)+}\right)_{j, j^{\prime} \in \Lambda_{j_{0}}^{+} \cup\left\{j_{0}, \bar{\jmath}_{0}\right\}}, \\
\text { where } V_{j j^{\prime}}^{\left(j_{0}\right)+}=\left\{\begin{array}{cc}
\delta_{j j^{\prime}} \mathbf{1}_{k_{j} k_{j^{\prime}}}-\mathrm{i} W_{j j^{\prime}}\left(D_{j^{\prime}} L_{j^{\prime}}\right), j^{\prime} \in\left\{j_{0}, \bar{\jmath}_{0}\right\} \\
-\mathrm{i} W_{j j^{\prime}}, & j^{\prime} \in \Lambda_{j_{0}}^{+}, \\
\text {and } c_{j_{0}}^{+}=2 \mathrm{i} \log (-1)^{\sum_{j \in \Lambda_{j_{0}}^{+}} k_{j}} .
\end{array}\right.
\end{gathered}
$$

We have to care about the ambiguities of the logarithm. Firstly there is a unique continuous choice of the logarithm such that for any $\tau$ we have $\lim _{\xi \rightarrow-\infty} u(\xi, \tau)=0$. Furthermore a standard inductive argument shows that there are unique choices of logarithms, such that the sum $\sum_{j_{0}: \Im\left(a_{j_{0}}\right) \geq 0} u_{a_{j_{0}}}^{+}(\xi, \tau)$ is close to $u(\xi, \tau)$ for sufficiently large $\tau$. To fix convenient branches of the logarithm, we have to start with the slowest cluster and choose the branch of the next faster cluster such that the graphs paste together.

The sign + in the notation $u_{a_{j}}^{+}$for $u_{a_{j_{0}}}$ indicates that the $j_{0}$-th cluster has suffered a phase-shift, which is due to its collision with all those clusters moving faster (i.e. by which the $j_{0}$-th cluster has been overtaken).

Proof. Consider the following intervals:

$$
\begin{aligned}
& I_{j_{0}}(\tau)=\left(\left(\lambda_{j_{0}}-\delta_{j_{0}}\right) \tau,\left(\lambda_{j_{0}}+\delta_{j_{0}}\right) \tau\right), \\
& 0<\delta_{j_{0}}<d_{j_{0}}:=\min _{j \neq j_{0}}\left|\lambda_{j}-\lambda_{j_{0}}\right|, \quad \forall j_{0}, \\
& I_{j_{1} j_{2}}(\tau)=\left(\left(\lambda_{j_{1}}+\delta_{j_{1} j_{2}}\right) \tau,\left(\lambda_{j_{2}}-\delta_{j_{1} j_{2}}\right) \tau\right), \quad 0<\delta_{j_{1} j_{2}}<\lambda_{j_{2}}-\lambda_{j_{1}}, \\
& \text { if } \lambda_{j_{1}}<\lambda_{j_{2}} \text { and there is no } \lambda_{j} \in\left(\lambda_{j_{1}}, \lambda_{j_{2}}\right) \quad \forall j_{1}, j_{2},
\end{aligned}
$$

and

$$
I_{-\infty}(\tau):=\left(-\infty,\left(\min _{j} \lambda_{j}-\delta_{-}\right) \tau\right), \quad I_{+\infty}(\tau):=\left(\left(\max _{j} \lambda_{j}+\delta_{+}\right) \tau,+\infty\right)
$$




$$
\text { for } \delta_{-}, \delta_{+}>0 \text {. }
$$

Geometrically, we cover $\mathbf{R}^{2}$ by sectors $S_{j_{0}}=\left\{(\xi, \tau): \xi \in I_{j_{0}}(\tau)\right\}$ (a small angle containing the line $\left.\xi=\lambda_{j_{0}} \tau\right), S_{j_{1} j_{2}}=\left\{(\xi, \tau): \xi \in I_{j_{1} j_{2}}(\tau)\right\}$ (angles between the lines $\xi=\lambda_{j_{1}} \tau$ and $\xi=\lambda_{j_{2}} \tau$ ), and $S_{-\infty}=\{(\xi, \tau)$ : $\left.\xi \in I_{-\infty}(\tau)\right\}, S_{+\infty}=\left\{(\xi, \tau): \xi \in I_{+\infty}(\tau)\right\}$ at the outside. Our aim is to find estimates in each of the sectors showing that the only contributions to the solution come from the $j_{0}$-th cluster in the sector $S_{j_{0}} \forall j_{0}$. More precisely, $u_{a_{j_{0}}}^{+}$dominates in the sector $S_{j_{0}}$, and all other terms under consideration decay to zero.

a) Now fix $j_{0}$ and take $\xi \in I_{j_{0}}(\tau)$.

For the entries $\ell_{j}^{(\kappa)}$ of $L_{j}$ and $\tilde{\ell}_{j}^{(\kappa)}$ of $L_{j}^{-1}$ (remember $L_{j}^{-1}$ is of the same structure as derived for $L_{j}$ in (6.4), (6.5), just start with $\ell_{j}^{-1}$ instead of $\ell_{j}$ ) we achieve

$$
\begin{aligned}
\left|\ell_{j}^{(\kappa)}(\xi, \tau)\right| & =q_{j, \kappa}(\xi, \tau) \exp \left(\quad \alpha_{j}\left(\xi-\lambda_{j} \tau\right)\right) \leq \\
& \leq Q_{j, \kappa}(\tau) \exp \left(-\widetilde{\alpha}_{j_{0}}\left(d_{j_{0}}-\delta_{j_{0}}\right) \tau\right), \quad j \in \Lambda_{j_{0}}^{-}, \\
\left|\tilde{\ell}_{j}^{(\kappa)}(\xi, \tau)\right| & =\tilde{q}_{j, \kappa}(\xi, \tau) \exp \left(-\alpha_{j}\left(\xi-\lambda_{j} \tau\right)\right) \leq \\
& \leq \tilde{Q}_{j, \kappa}(\tau) \exp \left(-\widetilde{\alpha}_{j_{0}}\left(d_{j_{0}}-\delta_{j_{0}}\right) \tau\right), \quad j \in \Lambda_{j_{0}}^{+},
\end{aligned}
$$

where $q_{j, \kappa}, \tilde{q}_{j, \kappa}$ are polynomials in $(\xi, \tau), Q_{j, \kappa}, \tilde{Q}_{j, \kappa}$ are polynomials in $\tau, \alpha_{j}=\sqrt{\frac{1-\omega_{j}^{2}}{1-\lambda_{j}^{2}}}>0$, and $\widetilde{\alpha}_{j_{0}}=\min _{j \neq j_{0}} \alpha_{j}$. Moreover,

$$
\left|\ell_{j_{0}}^{(\kappa)}(\xi, \tau)\right|,\left|\ell_{\bar{j}_{0}}^{(\kappa)}(\xi, \tau)\right| \leq Q_{j_{0}, \kappa}(\tau) \exp \left(\alpha_{j_{0}} \delta_{j_{0}} \tau\right)
$$

where $Q_{j_{0}, \kappa}$ again are polynomials.

Next, we verify that we can replace $p$ by $q^{+}=\operatorname{det}\left(\hat{V}_{j j^{\prime}}^{+}\right)_{j, j^{\prime}=1}^{k}$

$$
\text { with } \hat{V}_{j j^{\prime}}^{+}=\left\{\begin{array}{ll}
\delta_{j j^{\prime}} \mathbf{1}_{k_{j} k_{j^{\prime}}}-\mathrm{i} W_{j j^{\prime}}\left(L_{j^{\prime}} D_{j^{\prime}}\right), & j^{\prime} \in \Lambda_{j_{0}}^{-} \cup\left\{j_{0}, \bar{\jmath}_{0}\right\}, \\
\delta_{j j^{\prime}} \mathbf{1}_{k_{j} k_{j^{\prime}}}\left(L_{j^{\prime}} D_{j^{\prime}}\right)^{-1}-\mathrm{i} W_{j j^{\prime}}, & j^{\prime} \in \Lambda_{j_{0}}^{+}
\end{array} .\right.
$$

This is due to the fact that $p=q^{+} \prod_{j \in \Lambda_{j_{0}}^{+}} \operatorname{det}\left(L_{j} D_{j}\right)$, and the additional factor

$$
\prod_{j \in \Lambda_{j_{0}}^{+}} \operatorname{det}\left(D_{j}\right) \operatorname{det}\left(L_{j}\right)=\prod_{j \in \Lambda_{j_{0}}^{+}: \Im\left(a_{j}\right)=0}(-1)^{\frac{k_{j}\left(k_{j}+3\right)}{2}}\left(\ell_{j}^{(0)}\right)^{k_{j}} \prod_{j \in \Lambda_{j_{0}}^{+}: \Im\left(a_{j}\right)>0}\left|\ell_{j}^{(0)}\right|^{2 k_{j}}
$$


obviously does not change the solution.

Decomposing $\hat{V}_{j j^{\prime}}^{+}=V_{j j^{\prime}}^{+}+\Delta_{j j^{\prime}}^{+}$

$$
V_{j j^{\prime}}^{+}=\left\{\begin{array}{llll}
\delta_{j j^{\prime}} \mathbf{1}_{k_{j} k_{j^{\prime}},}, & & & j^{\prime} \in \Lambda_{j_{0}}^{-} \\
\delta_{j j^{\prime}} \mathbf{1}_{k_{j} k_{j^{\prime}}} & -\mathrm{i} W_{j j^{\prime}}\left(L_{j^{\prime}} D_{j^{\prime}}\right), & j^{\prime} \in\left\{j_{0}, \bar{\jmath}_{0}\right\} \\
& -\mathrm{i} W_{j j^{\prime}}, & j^{\prime} \in \Lambda_{j_{0}}^{+}
\end{array},\right.
$$

we observe that $\operatorname{det}\left(V_{j j^{\prime}}^{+}\right)_{j, j^{\prime}=1}^{k}=\operatorname{det}\left(V_{j j^{\prime}}^{+}\right)_{j, j^{\prime} \in \Lambda_{j_{0}}^{+} \cup\left\{j_{0}, \bar{J}_{0}\right\}}=$ $\operatorname{det}\left(V_{j j^{\prime}}^{\left(j_{0}\right)+}\right)_{j, j^{\prime} \in \Lambda_{j_{0}}^{+} \cup\left\{j_{0}, \bar{\jmath}_{0}\right\}}=p_{a_{j_{0}}}^{+}$by eliminating the entries without impact. From the above estimates $(6.11)-(6.13)$ for the entries and by Lemma 6.2 , we infer (to unify notations, $q_{a_{j_{0}}}^{+}:=p_{a_{j_{0}}}^{+}$)

$$
\left|q^{+}-q_{a_{j_{0}}}^{+}\right|=\left|\operatorname{det}\left(\hat{V}_{j j^{\prime}}^{+}\right)_{j, j^{\prime}=1}^{k}-\operatorname{det}\left(V_{j j^{\prime}}^{\left(j_{0}\right)+}\right)_{j, j^{\prime}}^{k}\right| \leq Q_{j_{0}}(\tau) \exp \left(-\gamma_{j_{0}} \tau\right)
$$

with a polynomial $Q_{j_{0}}$. Here, the constant $\gamma_{j_{0}}$ (see Lemma 6.2) is given by $\gamma_{j_{0}}=\widetilde{\alpha}_{j_{0}}\left(d_{j_{0}}-\delta_{j_{0}}\right)-K \alpha_{j_{0}} \delta_{j_{0}}, K:=\sum_{j} k_{j}$ the dimension of the whole matrix, and, to provide $\gamma_{j_{0}}>0$, we have to choose

$$
\delta_{j_{0}}<\frac{\widetilde{\alpha}_{j_{0}}}{\widetilde{\alpha}_{j_{0}}+K \alpha_{j_{0}}} d_{j_{0}} .
$$

We shall denote the contracted sector again by $I_{j_{0}}(\tau)$.

Repeating the arguments outlined above, we get analogous estimates for $p_{a_{j}}^{+}, j \in \Lambda_{j_{0}}^{+} \cup \Lambda_{j_{0}}^{-}$. Namely, if we replace $p_{a_{j}}^{+}$by $q_{a_{j}}^{+}=$ $\operatorname{det}\left(\hat{V}_{j^{\prime} j^{\prime \prime}}^{(j)+}\right)_{j^{\prime} j^{\prime \prime} \in \Lambda_{j}^{+} \cup\{j, \bar{j}\}}$, where

$$
\begin{gathered}
\hat{V}_{j^{\prime} j^{\prime \prime}}^{(j)+}=V_{j^{\prime} j^{\prime \prime}}^{(j)+} \text { if } j \in \Lambda_{j_{0}}^{-} \text {and } \\
\hat{V}_{j^{\prime} j^{\prime \prime}}^{(j)+}=\left\{\begin{array}{rl}
\delta_{j^{\prime} j^{\prime \prime}} \mathbf{1}_{k_{j^{\prime}} k_{j^{\prime \prime}}}\left(D_{j^{\prime \prime}} L_{j^{\prime \prime}}\right)^{-1}-\mathrm{i} W_{j^{\prime} j^{\prime \prime}}, & j^{\prime \prime} \in\{j, \bar{\jmath}\} \\
-\mathrm{i} W_{j^{\prime} j^{\prime \prime}}, & j^{\prime \prime} \in \Lambda_{j}^{+}
\end{array}, j \in \Lambda_{j_{0}}^{+},\right.
\end{gathered}
$$

again by Lemma 6.2 we observe

$$
\begin{array}{r}
\left|q_{a_{j}}^{+}-\operatorname{det}\left(-\mathrm{i} W_{j^{\prime} j^{\prime \prime}}\right)_{j^{\prime}, j^{\prime \prime} \in \Lambda_{j}^{+}}\right|<Q_{j} \exp \left(-\widetilde{\alpha}_{j_{0}}\left(d_{j_{0}}-\delta_{j_{0}}\right) \tau\right) \\
\text { for } j \in \Lambda_{j_{0}}^{-}, \\
\left|q_{a_{j}}^{+}-\operatorname{det}\left(-\mathrm{i} W_{j^{\prime} j^{\prime \prime}}\right)_{j^{\prime}, j^{\prime \prime} \in \Lambda_{j}^{+} \cup\{j, \bar{j}\}}\right|<Q_{j} \exp \left(-\widetilde{\alpha}_{j_{0}}\left(d_{j_{0}}-\delta_{j_{0}}\right) \tau\right) \\
\text { for } j \in \Lambda_{j_{0}}^{+}
\end{array}
$$


with constants $Q_{j}$.

Therefore, (6.14), (6.16), and (6.17) yield

$$
\begin{gathered}
\forall \varepsilon>0 \quad \exists \tau_{\varepsilon, j_{0}}: \quad\left|q^{+}-q_{a_{j_{0}}}^{+}\right|<\varepsilon \text { and }\left|q_{a_{j}}^{+}-C_{j}^{+}\right|<\varepsilon \forall j \neq j_{0} \\
\forall \tau \geq \tau_{\varepsilon, j_{0}} \text { uniformly in } S_{j_{0}},
\end{gathered}
$$

where

$$
\begin{aligned}
& C_{j}^{+}=\left\{\begin{array}{ll}
\operatorname{det}\left(-\mathrm{i} W_{j^{\prime} j^{\prime \prime}}\right)_{j^{\prime}, j^{\prime \prime} \in \Lambda_{j}^{+}}, & j \in \Lambda_{j_{0}}^{-} \\
\operatorname{det}\left(-\mathrm{i} W_{j^{\prime} j^{\prime \prime}}\right)_{j^{\prime}, j^{\prime \prime} \in \Lambda_{j}^{+} \cup\{j, \bar{\jmath}\}}, & j \in \Lambda_{j_{0}}^{+}
\end{array} \Longrightarrow\right. \\
& \Longrightarrow 2 \mathrm{i} \log \frac{C_{j}^{+}}{\overline{C_{j}^{+}}}= \begin{cases}c_{j}^{+}, & j \in \Lambda_{j_{0}}^{-} \\
c_{j}^{+}+\widetilde{c}_{j}^{+}, & j \in \Lambda_{j_{0}}^{+}\end{cases}
\end{aligned}
$$

with $\widetilde{c}_{j}^{+}=2 \mathrm{i} \log (-1)^{\sum_{j^{\prime} \in\{j, \bar{j}\}} k_{j^{\prime}}}$ (for all $j$ ). Since $\sum_{j \in \Lambda_{j_{0}}^{+}: \Im\left(a_{j}\right) \geq 0} \widetilde{c}_{j}^{+}=c_{j_{0}}^{+}$,

$$
\begin{gathered}
\left|u-\sum_{j: \Im\left(a_{j}\right) \geq 0} u_{a_{j}}^{+}\right|=\left|2 \mathrm{i} \log \frac{q^{+}}{\overline{q^{+}}}-\sum_{j: \Im\left(a_{j}\right) \geq 0}\left(2 \mathrm{i} \log \frac{q_{a_{j}}^{+}}{\overline{q_{a_{j}}^{+}}}-c_{j}^{+}\right)\right| \\
\leq\left|2 \mathrm{i} \log \frac{q^{+}}{\overline{q^{+}}}-2 \mathrm{i} \log \frac{q_{a_{j_{0}}}^{+}}{\overline{q_{a_{0}}^{+}}}\right|+\sum_{j \in \Lambda_{j_{0}}^{-}: \Im\left(a_{j}\right) \geq 0}\left|2 \mathrm{i} \log \frac{q_{a_{j}}^{+}}{\overline{q_{a_{j}}^{+}}}-c_{j}^{+}\right| \\
+\sum_{j \in \Lambda_{j_{0}}^{+}: \Im\left(a_{j}\right) \geq 0}\left|2 \mathrm{i} \log \frac{q_{a_{j}}^{+}}{\overline{q_{a_{j}}^{+}}}-\left(c_{j}^{+}+\widetilde{c}_{j}^{+}\right)\right| .
\end{gathered}
$$

Applying (6.18), we derive

$\forall \varepsilon>0 \quad \exists \tau_{\varepsilon, j_{0}}: \quad\left|u-\sum_{j: \Im\left(a_{j}\right) \geq 0} u_{a_{j}}^{+}\right|<\varepsilon \quad \forall \tau \geq \tau_{\varepsilon, j_{0}} \quad$ uniformly in $S_{j_{0}}$.

b) Secondly, fix $j_{1}, j_{2}$ with $\lambda_{j_{1}}<\lambda_{j_{2}}$ such that there is no $\lambda_{j} \in\left(\lambda_{j_{1}}, \lambda_{j_{2}}\right)$ and take $\xi \in I_{j_{1} j_{2}}(\tau)$.

In this situation, for the entries $\ell_{j}^{(\kappa)}$ of $L_{j}$ and $\tilde{\ell}_{j}^{(\kappa)}$ of $L_{j}^{-1}$ (remark $\left.\Lambda_{j_{2}}^{-} \cup \Lambda_{j_{1}}^{+}=\{1, \ldots, k\}\right)$ we use the estimates

$$
\begin{aligned}
\left|\ell_{j}^{(\kappa)}(\xi, \tau)\right| \leq P_{j, \kappa}(\tau) \exp \left(-\alpha \delta_{j_{1} j_{2}} \tau\right), \quad j \in \Lambda_{j_{2}}^{-}, \\
\left|\tilde{\ell}_{j}^{(\kappa)}(\xi, \tau)\right| \leq \tilde{P}_{j, \kappa}(\tau) \exp \left(-\alpha \delta_{j_{1} j_{2}} \tau\right), \quad j \in \Lambda_{j_{1}}^{+},
\end{aligned}
$$


where $P_{j, \kappa}, \tilde{P}_{j, \kappa}$ are polynomials in $\tau$ and $\alpha=\min _{j} \alpha_{j}$.

By the same argumentation as in a), replace $p$ by $q \pm \operatorname{det}\left(\hat{V}_{j j^{\prime}}^{+}\right)_{j, j^{\prime}=1}^{k}$,

$$
\hat{V}_{j j^{\prime}}^{+}=\left\{\begin{array}{ll}
\delta_{j j^{\prime}} \mathbf{1}_{k_{j} k_{j^{\prime}}}-\mathrm{i} W_{j j^{\prime}}\left(L_{j^{\prime}} D_{j^{\prime}}\right), & j^{\prime} \in \Lambda_{j_{2}}^{-} \\
\delta_{j j^{\prime}} \mathbf{1}_{k_{j} k_{j^{\prime}}}\left(L_{j^{\prime}} D_{j^{\prime}}\right)^{-1}-\mathrm{i} W_{j j^{\prime}}, & j^{\prime} \in \Lambda_{j_{1}}^{+}
\end{array},\right.
$$

and $p_{a_{j}}^{+}$by $q_{a_{j}}^{+}=\operatorname{det}\left(\hat{V}_{j^{\prime} j^{\prime \prime}}^{(j)+}\right)_{j^{\prime}, j^{\prime \prime} \Lambda_{j}^{+} \cup\{j, \bar{\jmath}\}}$,

$$
\begin{gathered}
\hat{V}_{j^{\prime} j^{\prime \prime}}^{(j)+}=V_{j^{\prime} j^{\prime \prime}}^{(j)+} \text { if } j \in \Lambda_{j_{2}}^{-} \text {and } \hat{V}_{j^{\prime} j^{\prime \prime}}^{(j)+}= \\
=\left\{\begin{array}{rl}
\delta_{j^{\prime} j^{\prime \prime}} \mathbf{1}_{k_{j^{\prime}} k_{j^{\prime \prime}}}\left(L_{j^{\prime \prime}} D_{j^{\prime \prime}}\right)^{-1}-\mathrm{i} W_{j^{\prime} j^{\prime \prime}}, & j^{\prime \prime} \in\{j, \bar{\jmath}\} \\
-\mathrm{i} W_{j^{\prime} j^{\prime \prime}}, & j^{\prime \prime} \in \Lambda_{j}^{+}
\end{array}, j \in \Lambda_{j_{1}}^{+} .\right.
\end{gathered}
$$

Using the estimates (6.19), (6.20), and Lemma 6.2 once more, we conclude

$$
\begin{aligned}
& \forall \varepsilon>0 \quad \exists \tau_{\varepsilon, j_{1} j_{2}}: \quad\left|q^{+}-B^{+}\right|<\varepsilon \text { and }\left|q_{a_{j}}^{+}-B_{j}^{+}\right|<\varepsilon \forall j \quad \forall \tau \geq \tau_{\varepsilon, j_{1} j_{2}} \\
& \text { uniformly in } S_{j_{1} j_{2}} \text {, } \\
& B^{+}=\operatorname{det}\left(-\mathrm{i} W_{j j^{\prime}}\right)_{j, j^{\prime} \in \Lambda_{j_{1}}^{+}}, B_{j}^{+}=\left\{\begin{array}{ll}
\operatorname{det}\left(-\mathrm{i} W_{j^{\prime} j^{\prime \prime}}\right)_{j^{\prime}, j^{\prime \prime} \in \Lambda_{j}^{+}}, & j \in \Lambda_{j_{2}}^{-} \\
\operatorname{det}\left(-\mathrm{i} W_{j^{\prime} j^{\prime \prime}}\right)_{j^{\prime}, j^{\prime \prime} \in \Lambda_{j}^{+} \cup\{j, \bar{j}\}}, & j \in \Lambda_{j_{1}}^{+}
\end{array} .\right.
\end{aligned}
$$

Following still the line of argumentation of a) and using the same notation for the constants $c_{j}^{+}, \widetilde{c}_{j}^{+}$as in $\left.\mathbf{a}\right)$, we get

$$
\begin{array}{r}
\left|u-\sum_{j: \Im\left(a_{j}\right) \geq 0} u_{a_{j}}^{+}\right| \leq\left|2 \mathrm{i} \log \frac{q^{+}}{\overline{q^{+}}}-c_{j_{1}}^{+}\right|+\sum_{j \in \Lambda_{j_{2}}^{-}: \Im\left(a_{j}\right) \geq 0}\left|2 \mathrm{i} \log \frac{q_{a_{j}}^{+}}{q_{a_{j}}^{+}}-c_{j}^{+}\right| \\
+\sum_{j \in \Lambda_{j_{1}}^{+}: \Im\left(a_{j}\right) \geq 0}\left|2 \mathrm{i} \log \frac{q_{a_{j}}^{+}}{\overline{q_{a_{j}}^{+}}}-\left(c_{j}^{+}+\widetilde{c}_{j}^{+}\right)\right| .
\end{array}
$$

Thus, by (6.21), we proved

$\forall \varepsilon>0 \quad \exists \tau_{\varepsilon, j_{1} j_{2}}: \quad\left|u-\sum_{j: \Im\left(a_{j}\right) \geq 0} u_{a_{j}}^{+}\right|<\varepsilon \quad \forall \tau \geq \tau_{\varepsilon, j_{1} j_{2}} \quad$ uniformly in $S_{j_{1} j_{2}}$.

c) Next, consider $\xi \in I_{-\infty}(\tau)$ or $\xi \in I_{+\infty}(\tau)$. 
Repeating the arguments of $\mathbf{b}$ ), we obtain that for all $\varepsilon>0$ there exists a $\tau_{\varepsilon,-}$ such that $\left|p^{+}-1\right|<\varepsilon$ and $\left|p_{a_{j}}^{+}-\operatorname{det}\left(-\mathrm{i} W_{j^{\prime} j^{\prime \prime}}\right)_{j^{\prime}, j^{\prime \prime} \in \Lambda_{j}^{+}}\right|<\varepsilon$ $(\forall j)$ for all $\tau \geq \tau_{\varepsilon,-}$ uniformly in $S_{-\infty}$. This yields immediately

$\forall \varepsilon>0 \quad \exists \tau_{\varepsilon,-}: \quad|u|<\varepsilon$ and $\left|u_{a_{j}}^{+}\right|<\varepsilon \forall j \quad \forall \tau \geq \tau_{\varepsilon,-}$ uniformly in $S_{-\infty}$.

Moreover, replacing $p$ by $q^{+}, p_{a_{j}}^{+}$by $q_{a_{j}}^{+}$(i.e. exchanging $L_{j}$ for $L_{j}^{-1}$ ), we observe that for all $\varepsilon>0$ there exists a $\tau_{\varepsilon,+}$ such that $\mid q^{+}-\operatorname{det}(-$ $\left.\mathrm{i} W_{j j^{\prime}}\right)_{j, j^{\prime}=1}^{k} \mid<\varepsilon$ and $\left|q_{a_{j}}^{+}-\operatorname{det}\left(-\mathrm{i} W_{j^{\prime} j^{\prime \prime}}\right)_{j^{\prime}, j^{\prime \prime} \in \Lambda_{j}^{+} \cup\{j, \bar{j}\}}\right|<\varepsilon(\forall j)$ for all $\tau \geq \tau_{\varepsilon,+}$ uniformly in $S_{+\infty}$. Hence

$$
\begin{aligned}
& \forall \varepsilon>0 \quad \exists \tau_{\varepsilon,+}: \quad\left|u-2 \mathrm{i} \log (-1)^{\sum_{j=1}^{k} k_{j}}\right|<\varepsilon \text { and } \\
& \left|u_{a_{j}}^{+}-2 \mathrm{i} \log (-1)^{\sum_{j^{\prime} \in\{j, \bar{j}\}} k_{j^{\prime}}}\right|<\varepsilon \forall j \quad \forall \tau \geq \tau_{\varepsilon,+} \\
& \text { uniformly in } S_{+\infty} \text {. }
\end{aligned}
$$

d) At last, we check that the constants $\delta_{j_{0}}, \delta_{j_{1} j_{2}}$, and $\delta_{-}, \delta_{+}$can be chosen such that the intervals $I_{j_{0}}(\tau)\left(\forall j_{0}\right), I_{j_{1} j_{2}}(\tau)\left(\forall j_{1}, j_{2}\right)$ and $I_{-\infty}(\tau)$, $I_{+\infty}(\tau)$ cover $\mathbf{R}(\forall \tau)$.

For $\varepsilon>0$ let $\tau_{\varepsilon}$ be sufficiently large such that the estimates in a) c) hold simultaneously for $\tau \geq \tau_{\varepsilon}$. Thus

$$
\left|u-\sum_{j: \Im\left(a_{j}\right) \geq 0} u_{a_{j}}^{+}\right|<\varepsilon \quad \forall \tau \geq \tau_{\varepsilon} \quad \text { uniformly in } \xi \in \mathbf{R} .
$$

\section{Step 2.}

For later use we have to calculate a certain determinant.

Lemma 6.3. With the usual convention that empty products equal one,

$$
\operatorname{det}\left(\prod_{\bar{\kappa}=1}^{\kappa^{\prime}-1}[(a-(\kappa-1))-\bar{\kappa}]\right)_{\kappa, \kappa^{\prime}=1}^{m}=(-1)^{\frac{m(m+3)}{2}} \prod_{\kappa=1}^{m-1} \kappa ! \text {. }
$$

Proof. If we multiply the $\kappa^{\prime}-1$-th column by $\left[(a-(m-1))-\left(\kappa^{\prime}-1\right)\right]$ and subtract it from the $\kappa^{\prime}$-th column for $\kappa^{\prime}=m, \ldots, 2$, we get

$$
\operatorname{det}\left(\prod_{\bar{\kappa}=1}^{\kappa^{\prime}-1}[(a-(\kappa-1))-\bar{\kappa}]\right)_{\kappa, \kappa^{\prime}=1}^{m}=
$$




$$
=\operatorname{det}\left(\delta_{1 \kappa^{\prime}}+\left(1-\delta_{1 \kappa^{\prime}}\right)(m-\kappa) \prod_{\bar{\kappa}=1}^{\left(\kappa^{\prime}-1\right)-1}[(a-(\kappa-1))-\bar{\kappa}]\right)_{\kappa, \kappa^{\prime}=1}^{m} .
$$

Expanding the latter determinant with respect to the $m$-th row and extracting the factor $(m-\kappa)$ from all other rows $\kappa<m$, we attain

$$
=(-1)^{m+1} \prod_{\kappa=1}^{m-1}(m-\kappa) \cdot \operatorname{det}\left(\prod_{\bar{\kappa}=1}^{\kappa^{\prime}-1}[(a-(\kappa-1))-\bar{\kappa}]\right)_{\kappa, \kappa^{\prime}=1}^{m-1},
$$

and the assertion now follows by induction.

Corollary 6.4. Let $n \in \mathbf{N}$ and define $F=\left(\operatorname{fak}_{\kappa \kappa^{\prime}}\right)_{\kappa, \kappa^{\prime}=1}^{m}$ by fak f $_{\kappa \kappa^{\prime}}:=0$ for $\kappa+\kappa^{\prime}>n+1$ and fak $_{\kappa \kappa^{\prime}}:=\frac{1}{\left[n-\left(\kappa+\kappa^{\prime}-1\right)\right] !}$ for $\kappa+\kappa^{\prime} \leq n+1$. Then

$$
\operatorname{det}(F)=(-1)^{\frac{m(m+3)}{2}}\left(\prod_{\kappa=1}^{m-1} \kappa !\right)\left(\prod_{\kappa=1}^{m}(n-\kappa) !\right)^{-1} .
$$

Proof. For $\kappa+\kappa^{\prime} \leq n+1$,

$$
\begin{gathered}
(n-\kappa) ! \mathrm{fak}_{\kappa \kappa^{\prime}}=\frac{(n-\kappa) !}{\left[(n-\kappa)-\left(\kappa^{\prime}-1\right)\right] !}= \\
=\prod_{\bar{\kappa}=0}^{\kappa^{\prime}-2}[(n-\kappa)-\bar{\kappa}]=\prod_{\bar{\kappa}=1}^{\kappa^{\prime}-1}[(n-(\kappa-1))-\bar{\kappa}] .
\end{gathered}
$$

Since the product on the right-hand side vanishes for $\kappa+\kappa^{\prime}>n+1$ (the factor 0 appears whenever $\left.\bar{\kappa}=(n+1)-\kappa<\kappa^{\prime}\right)$, we infer $(n-\kappa)$ ! fak ${\text { f } \kappa^{\prime}}=$ $\prod_{\bar{\kappa}=1}^{\kappa^{\prime}-1}[(n-(\kappa-1))-\bar{\kappa}] \quad \forall \kappa, \kappa^{\prime}$. Therefore,

$$
\begin{aligned}
\operatorname{det}(F) & =\operatorname{det}\left(\frac{1}{(n-\kappa) !} \prod_{\bar{\kappa}=1}^{\kappa^{\prime}-1}[(n-(\kappa-1))-\bar{\kappa}]\right)_{\kappa, \kappa^{\prime}=1}^{m}= \\
& =(-1)^{\frac{m(m+3)}{2}}\left(\prod_{\kappa=1}^{m-1} \kappa !\right)\left(\prod_{\kappa=1}^{m}(n-\kappa) !\right)^{-1},
\end{aligned}
$$

the latter by extracting the factor $\frac{1}{(n-\kappa) !}$ from the $\kappa$-th row $(\forall \kappa)$ and applying Lemma 6.3. 
Assertion. Now we fix the $j_{0}$-th cluster

$$
u_{a_{j_{0}}}^{+}=2 \mathrm{i} \log \frac{p_{a_{j_{0}}}^{+}}{\overline{p_{a_{j_{0}}}^{+}}}-c_{j_{0}}^{+}, \quad \text { where } c_{j_{0}^{+}}=2 \mathrm{i} \log (-1)^{\sum_{j \in \Lambda_{j_{0}}^{+}}^{k_{j}}}
$$

To simplify arguments, we will first treat the case that the $j_{0}$-th cluster corresponds to a real eigenvalue $a_{j_{0}} \in \mathbf{R}$ separately and indicate thereafter the purely notational changes necessary for clusters corresponding to a pair of complex conjugate eigenvalues $a_{\bar{\jmath}_{0}}=\bar{a}_{j_{0}}$.

We show

$$
u_{a_{j_{0}}}^{+}(\xi, \tau) \approx \sum_{\kappa_{0}} u_{a_{j_{0}, \kappa_{0}}}^{+}(\xi, \tau)
$$

where $\kappa_{0}=-\left(k_{j_{0}}-1\right)+2 \kappa_{0}^{\prime}$ for $\kappa_{0}^{\prime}=0, \ldots, k_{j_{0}}-1$ (vice versa, $\kappa_{0}^{\prime}=$ $\left.\frac{\left(k_{j_{0}}-1\right)+\kappa_{0}}{2}\right)$. The participating solitons/antisolitons $u_{a_{j_{0}}, \kappa_{0}}^{+}(\xi, \tau)$ are defined along the curves

$$
\begin{gathered}
\mathcal{A}_{a_{j_{0}, \kappa_{0}}}(\xi, \tau)=\frac{\xi-\lambda_{j_{0}} \tau}{\sqrt{1-\lambda_{j_{0}}^{2}}}-\kappa_{0} \log |\tau|+\left(\varphi_{j_{0}}+\varphi_{j_{0}}^{+}+\varphi_{j_{0}, \kappa_{0}}^{+}\right) \\
e^{\varphi_{j_{0}}}=\frac{\exp \left(f_{j_{0}}\left(a_{j_{0}}\right)\right)}{\left(2 a_{j_{0}}\right)^{k_{j_{0}}}}, \quad e^{\varphi_{j_{0}}^{+}}=\prod_{j \in \Lambda_{j_{0}}^{+}}\left(\frac{a_{j}-a_{j_{0}}}{a_{j}+a_{j_{0}}}\right)^{2 k_{j}}, \\
e^{\varphi_{j_{0}, \kappa_{0}}^{+}}=\frac{\left(\frac{\left(k_{j_{0}}-1\right)+\kappa_{0}}{2}\right) !}{\left(\frac{\left(k_{j_{0}}-1\right)-\kappa_{0}}{2}\right) !}\left(\frac{4 a_{j_{0}}}{1+a_{j_{0}}^{2}}\right)^{-\kappa_{0}}
\end{gathered}
$$

by $\quad u_{a_{j_{0}, \kappa_{0}}}^{+}=2 \mathrm{i} \log \frac{p_{a_{j_{0}, \kappa_{0}}^{+}}^{+}}{\overline{p_{a_{j_{0}}, \kappa_{0}}^{+}}} \quad$ and

$$
p_{a_{j_{0}}, \kappa_{0}}^{+}(\xi, \tau)=1-\mathrm{i}(-1) \frac{\left(k_{j_{0}}-1\right)-\kappa_{0}}{2} \exp \left(\mathcal{A}_{a_{j_{0}, \kappa_{0}}}(\xi, \tau)\right)
$$

To care about the ambiguities of the logarithm, we proceed as indicated in Step 1. 


\section{Proof.}

(1) At first, we derive estimates for the entries of $L_{j_{0}}$ on logarithmic curves $\xi=\Gamma_{\rho}(\tau)$ defined by

$$
\Gamma_{\rho}(\tau)=\lambda_{j_{0}} \tau+\sqrt{1-\lambda_{j_{0}}^{2}} \cdot(\rho \log |\tau|) \quad \text { for } \rho \in \mathbf{R} .
$$

Obviously,

$$
\xi=\Gamma_{\rho}(\tau) \Longleftrightarrow \frac{\xi-\lambda_{j_{0}} \tau}{\sqrt{1-\lambda_{j_{0}}^{2}}}=\rho \log |\tau| .
$$

On this curve, the following estimate holds,

$$
\begin{aligned}
& \ell_{j_{0}}^{(\kappa)}\left(\Gamma_{\rho}(\tau), \tau\right)= \\
& \quad=\frac{\left(-d_{j_{0}}\right)^{\kappa}}{\kappa !} \exp \left(f_{j_{0}}\left(a_{j_{0}}\right)\right) \tau^{\kappa}|\tau|^{\rho} \cdot\left[1+\mathcal{O}\left(\frac{\log |\tau|}{\tau}\right)\right] \quad \forall \kappa,
\end{aligned}
$$

with $d_{j_{0}}=\frac{2}{1+a_{j_{0}}^{2}}$.

For the proof, remember $a_{j_{0}}=\sqrt{\frac{1+\lambda_{j_{0}}}{1-\lambda_{j_{0}}}}$. Thus, from (6.6), (6.7), we infer

$$
\ell_{j_{0}}\left(\Gamma_{\rho}(\tau), \tau\right)=
$$

$$
=|\tau|^{\rho} \exp \left(f_{j_{0}}\left(a_{j_{0}}\right)\right) \text { and } p_{j_{0}}^{(1)}\left(\Gamma_{\rho}(\tau), \tau\right)=-d_{j_{0}} \tau+\mathcal{O}(\log |\tau|) .
$$

By the recursion formula $(6.5)$ and the definition of $\ell_{j_{0}}^{(\kappa)}$, the desired estimates follow easily.

(2) Next we investigate the behaviour of the determinant $p_{a_{j_{0}}}^{+}=$ $\operatorname{det}\left(V_{j j^{\prime}}\right)_{j, j^{\prime} \in \Lambda_{j_{0}}^{+} \cup\left\{j_{0}, \bar{j}_{0}\right\}}$ (for brevity, we shall omit the upper indices) on the curve (6.23). Since its structure

$$
\begin{aligned}
V_{j j^{\prime}}=\widehat{\delta}_{j j^{\prime}} \mathbf{1}_{k_{j} k_{j^{\prime}}}+\widehat{V}_{j j^{\prime}} & \text { with } \quad \widehat{V}_{j j^{\prime}}= \begin{cases}-\mathrm{i} W_{j j^{\prime}}\left(D_{j^{\prime}} L_{j^{\prime}}\right), & j^{\prime}=j_{0} \\
-\mathrm{i} W_{j j^{\prime}}, & j^{\prime} \in \Lambda_{j 0}^{+}\end{cases} \\
\text {and } \quad \widehat{\delta}_{j j^{\prime}} & =\left\{\begin{array}{lll}
1 & \text { if } j, j^{\prime}=j_{0} \\
0 & \text { else }
\end{array}\right.
\end{aligned}
$$


is similar to that of $1+T$ with $T=\left(t_{j j^{\prime}}\right)_{j j^{\prime}=1}^{n}$, we expand it by analogy with the well-known expansion

$$
\operatorname{det}(1+T)=1+\sum_{m=1}^{n} \sum_{\sigma_{1}<\ldots<\sigma_{m}} \operatorname{det}\left(\begin{array}{ccc}
t_{\sigma_{1} \sigma_{1}} & \cdots & t_{\sigma_{1} \sigma_{m}} \\
\ldots \ldots & \ldots & \ldots \\
t_{\sigma_{m} \sigma_{1}} & \cdots & t_{\sigma_{m} \sigma_{m}}
\end{array}\right) .
$$

To this end, we need some additional notations:

$$
\text { Let } T=\left(T_{j j^{\prime}}\right)_{j j^{\prime}} \text { with } T_{j j^{\prime}}=\left(T_{j j^{\prime}}^{\left(\kappa \kappa^{\prime}\right)}\right)_{\substack{\kappa=1, \ldots, k_{j} \\ \kappa^{\prime}=1, \ldots, k_{j^{\prime}}}} \text {. For an index set }
$$

$J \subseteq\left\{1, \ldots, k_{j_{0}}\right\}$, we denote by $T[J]$ the matrix built from $T$ restricting the index sets in the following way:

$$
\kappa \in J \quad \text { if } j=j_{0} \quad \text { and } \quad \kappa^{\prime} \in J \quad \text { if } j^{\prime}=j_{0} .
$$

With this notations,

$$
\operatorname{det}(V)=\operatorname{det}\left(\widehat{V}_{j j^{\prime}}\right)_{j, j^{\prime} \in \Lambda_{j_{0}}^{+}}+\sum_{m=1}^{k_{j_{0}}} \sum_{\sigma_{1}<\ldots<\sigma_{m}} \operatorname{det}\left(\widehat{V}\left[\left\{\sigma_{1}, \ldots, \sigma_{m}\right\}\right]\right),
$$

for $V=\left(V_{j j^{\prime}}\right)_{j, j^{\prime} \in \Lambda_{j 0}^{+} \cup\{j 0\}}$ and $\widehat{V}=\left(\widehat{V}_{j j^{\prime}}\right)_{j, j^{\prime} \in \Lambda_{j_{0}}^{+} \cup\left\{j_{0}\right\}}$.

To take a closer look at each summand, we write down the matrices $\widehat{V}_{j j_{0}}$ explicetly:

$\widehat{V}_{j j_{0}}=-\mathrm{i} W_{j j_{0}}\left(D_{j_{0}} L_{j_{0}}\right)=\left(-\mathrm{i} \sum_{\kappa=1}^{k_{j_{0}}-j_{0}^{\prime}+1} W_{j j_{0}}^{(j \kappa)} \ell_{j_{0}}^{\left(k_{j_{0}}-j_{0}^{\prime}+1-\kappa\right)}\right)_{\substack{j^{\prime}=1, \ldots, k_{j} \\ j_{0}^{\prime}=1, \ldots, k_{0}}}$.

Expanding once more, we obtain

$$
\begin{aligned}
\operatorname{det}\left(\widehat{V}\left[\left\{\sigma_{1}, \ldots, \sigma_{m}\right\}\right]\right)=\sum_{\sigma_{1}^{\prime}=1}^{k_{j_{0}}-\sigma_{1}+1} \sum_{\sigma_{m}^{\prime}=1}^{k_{j_{0}}-\sigma_{m}+1} \ell_{j_{0}}^{\left(k_{j_{0}}+1-\sigma_{1}-\sigma_{1}^{\prime}\right)} \ldots \ell_{j_{0}}^{\left(k_{j_{0}}+1-\sigma_{m}-\sigma_{m}^{\prime}\right)} . \\
\cdot \operatorname{det}\left(-\mathrm{i} W\left[\left\{\sigma_{1}^{\prime}, \ldots, \sigma_{m}^{\prime}\right\}\right]\right)
\end{aligned}
$$

where again $-\mathrm{i} W=\left(-\mathrm{i} W_{j j^{\prime}}\right)_{j, j^{\prime} \in \Lambda_{j 0}^{+} \cup\left\{j_{0}\right\}}$. 
According to estimate (6.24),

$$
\begin{gathered}
\prod_{\kappa=1}^{m} \ell_{j_{0}}^{\left(k_{j_{0}}+1-\sigma_{\kappa}-\sigma_{\kappa}^{\prime}\right)}=\frac{\left(-d_{j_{0}}\right)^{\sum_{\kappa=1}^{m}\left(k_{j_{0}}+1-\sigma_{\kappa}-\sigma_{\kappa}^{\prime}\right)}}{\prod_{\kappa=1}^{m}\left(k_{j_{0}}+1-\sigma_{\kappa}-\sigma_{\kappa}^{\prime}\right) !}\left(\exp \left(f_{j_{0}}\left(a_{j_{0}}\right)\right)\right)^{m} . \\
\cdot|\tau|^{\rho m} \cdot(\tau)^{\sum_{\kappa=1}^{m}\left(k_{j_{0}}+1-\sigma_{\kappa}-\sigma_{\kappa}^{\prime}\right)} \cdot\left[1+\mathcal{O}\left(\frac{\log |\tau|}{\tau}\right)\right] .
\end{gathered}
$$

The values of $\sigma_{\kappa}, \sigma_{\kappa}^{\prime}(\kappa=1, \ldots, m)$ which maximize the power of $\tau$ in this expression can be determined precisely:

For fixed tuples $\left(\sigma_{1}, \ldots, \sigma_{m}\right)$, the corresponding sum $\sum_{\kappa=1}^{m}\left(k_{j_{0}}+1-\right.$ $\left.\sigma_{\kappa}-\sigma_{\kappa}^{\prime}\right)$, which is responsible for the power of $\tau$, is maximal if and only if $\sum_{\kappa=1}^{m} \sigma_{\kappa}^{\prime}$ is minimal. In addition, all $\sigma_{\kappa}^{\prime}$ have to be different, otherwise the determinant in (6.26) contains linearly dependent columns and hence vanishes. Thus, the right condition is

$\sigma_{\kappa}^{\prime}=\pi(\kappa) \quad(\kappa=1, \ldots, m) \quad$ with $\pi \in S_{m}$ (permutations on $m$ figures).

In the sum over all possible (ordered) tuples $\left(\sigma_{1}, \ldots, \sigma_{m}\right)$, the term of maximal order in $\tau$ corresponds to the choice $\sigma_{\kappa}=\kappa(\kappa=1, \ldots, m)$, hence the maximal contribution to the power of $\tau$ is

$$
\sum_{\kappa=1}^{m}\left(k_{j_{0}}+1-(\kappa+\pi(\kappa))\right)=\left(k_{j_{0}}-m\right) m .
$$

In addition, we have to examine the range of the summation index, $\kappa+\pi(\kappa)=\sigma_{\kappa}+\sigma_{\kappa}^{\prime} \leq k_{j_{0}}+1$. For this we replace the faculties $1 /\left(k_{j_{0}}+\right.$ $\left.1-\sigma_{\kappa}-\sigma_{\kappa}^{\prime}\right)$ ! in (6.27) by zero according to the rule

$$
\begin{gathered}
\operatorname{fak}_{\kappa \kappa^{\prime}}=\frac{1}{\left(k_{j_{0}}+1-\left(\kappa+\kappa^{\prime}\right)\right) !} \text { for } \kappa+\kappa^{\prime} \leq k_{j_{0}}+1 \text { and } \\
\operatorname{fak}_{\kappa \kappa^{\prime}}=0 \text { for } \kappa+\kappa^{\prime}>k_{j_{0}}+1 .
\end{gathered}
$$

Combining those conditions with (6.26), (6.27), we get

$$
\begin{gathered}
\sum_{\sigma_{1}<\ldots<\sigma_{m}} \operatorname{det}\left(\widehat{V}\left[\left\{\sigma_{1}, \ldots, \sigma_{m}\right\}\right]\right)=H_{m} \cdot\left(\left(-d_{j_{0}}\right)^{\left(k_{j_{0}}-m\right)} \exp \left(f_{j_{0}}\left(a_{j_{0}}\right)\right)\right)^{m} \\
\cdot|\tau|^{\rho m}(\tau)^{\left(k_{j_{0}}-m\right) m} \cdot\left[1+\mathcal{O}\left(\frac{\log |\tau|}{\tau}\right)\right]
\end{gathered}
$$




$$
\text { with } \begin{aligned}
& H_{m}=\sum_{\pi \in S_{m}} \prod_{\kappa=1}^{m} \mathrm{fak}_{\kappa \pi(\kappa)} \cdot \operatorname{det}(-\mathrm{i} W[\{\pi(1), \ldots, \pi(m)\}]) \\
&=\sum_{\pi \in S_{m}} \operatorname{sgn}(\pi) \prod_{\kappa=1}^{m} \mathrm{fak}_{\kappa \pi(\kappa)} \cdot \operatorname{det}(-\mathrm{i} W[\{1, \ldots, m\}]) \\
&=(-\mathrm{i})^{\sum_{j \in \Lambda_{j_{0}}^{+}}^{k_{j}}}(-\mathrm{i})^{m} \operatorname{det}\left(\mathrm{fak}_{\kappa \kappa^{\prime}}\right)_{\kappa, \kappa^{\prime}=1}^{m} \operatorname{det}(W[\{1, \ldots, m\}]) .
\end{aligned}
$$

By Theorem 6.1, we observe

$$
\begin{gathered}
\operatorname{det}(W[\{1, \ldots, m\}])=\Omega_{m}^{+} \cdot \operatorname{det}\left(W_{j j^{\prime}}\right)_{j, j^{\prime} \in \Lambda_{j_{0}}^{+}} \\
\text {for } \Omega_{m}^{+}=\left[\frac{1}{2 a_{j_{0}}}\right]^{m^{2}} \prod_{j \in \Lambda_{j_{0}}^{+}}\left[\frac{a_{j}-a_{j_{0}}}{a_{j}+a_{j_{0}}}\right]^{2 m k_{j}},
\end{gathered}
$$

and by Corollary 6.4 we get

$$
\operatorname{det}\left(\operatorname{fak}_{\kappa \kappa^{\prime}}\right)_{\kappa, \kappa^{\prime}=1}^{m}=(-1)^{\frac{m(m+3)}{2}}\left(\prod_{\kappa=1}^{m-1} \kappa !\right)\left(\prod_{\kappa=1}^{m}(n-\kappa) !\right)^{-1} .
$$

Hence $H_{m}=(-\mathrm{i})^{\sum_{j \in \Lambda_{j}^{+}} k_{j}} \operatorname{det}\left(W_{j j^{\prime}}\right)_{j, j^{\prime} \in \Lambda_{j_{0}}^{+}}$.

$$
\cdot(-1)^{\frac{m(m+3)}{2}} \frac{\prod_{\kappa=1}^{m-1} \kappa !}{\prod_{\kappa=1}^{m}\left(k_{j_{0}}-\kappa\right) !} \cdot(-\mathrm{i})^{m} \Omega_{m}^{+}
$$

Finally, we insert $(6.28),(6.31)$ in (6.25). This yields

$$
\begin{aligned}
& p_{a_{j_{0}}}^{+}\left(\Gamma_{\rho}(\tau), \tau\right)=(-\mathrm{i})^{\sum_{j \in \Lambda_{j_{0}}^{+}} k_{j}} X^{+} . \\
& \quad\left(1+\sum_{m=1}^{k_{j_{0}}}(-\mathrm{i})^{m} \Omega_{m}^{+} X_{m}^{+}|\tau|^{\rho m}(\tau)^{\left(k_{j_{0}}-m\right) m}\left[1+\mathcal{O}\left(\frac{\log |\tau|}{\tau}\right)\right]\right)
\end{aligned}
$$

with

$$
X_{m}^{+}=(-1)^{\frac{m(m+3)}{2}} \frac{\prod_{\kappa=1}^{m-1} \kappa !}{\prod_{\kappa=1}^{m}\left(k_{j_{0}}-\kappa\right) !}\left(\left(-d_{j_{0}}\right)^{k_{j_{0}}-m} \exp \left(f_{j_{0}}\left(a_{j_{0}}\right)\right)\right)^{m}
$$




$$
\text { and } X^{+}:=\operatorname{det}\left(W_{j j^{\prime}}\right)_{j, j^{\prime} \in \Lambda_{j_{0}}^{+}}
$$

Remark. To carry out the asymptotic analysis for $\tau \longrightarrow-\infty$, it is more convenient to consider $-\tau \longrightarrow+\infty$ and use $\widehat{X}_{m}^{-}=(-1)^{\left(k_{j_{0}}-m\right) m} X_{m}^{-}$ instead of $X_{m}^{-}$.

(3) In what follows, $\kappa$ is always an element of the index set specified in the assertion, and $\kappa^{\prime}$ is associated to $\kappa$ as described there.

Consider the intervals

$$
\begin{gathered}
I_{\kappa_{0}}(\tau):=\left(\lambda_{j_{0}} \tau+\sqrt{1-\lambda_{j_{0}}^{2}}\left(\left(\kappa_{0}-\delta_{\kappa_{0}}\right) \log |\tau|\right), \lambda_{j_{0}} \tau+\sqrt{1-\lambda_{j_{0}}^{2}}\left(\left(\kappa_{0}+\delta_{\kappa_{0}}\right) \log |\tau|\right)\right) \\
0<\delta_{\kappa_{0}}<\frac{1}{2}, \quad \forall \kappa_{0}, \quad(6.35) \\
I_{\kappa_{1} \kappa_{2}}(\tau):=\left(\lambda_{j_{0}} \tau+\sqrt{1-\lambda_{j_{0}}^{2}}\left(\left(\kappa_{1}+\delta_{\kappa_{1} \kappa_{2}}\right) \log |\tau|\right), \lambda_{j_{0}} \tau+\sqrt{1-\lambda_{j_{0}}^{2}}\left(\left(\kappa_{2}-\delta_{\kappa_{1} \kappa_{2}}\right) \log |\tau|\right)\right),
\end{gathered}
$$

$0<\delta_{\kappa_{1} \kappa_{2}}<\kappa_{2}-\kappa_{1}$, if $\kappa_{1}<\kappa_{2}$ and there is no $\kappa \in\left(\kappa_{1}, \kappa_{2}\right), \quad \forall \kappa_{1}, \kappa_{2}$, and

$$
\begin{gathered}
I_{-\infty}(\tau)=\left(-\infty, \lambda_{j_{0}} \tau+\sqrt{1-\lambda_{j_{0}}^{2}}\left(\left(\min \kappa-\delta_{-}\right) \log |\tau|\right)\right) \\
I_{+\infty}(\tau)=\left(\lambda_{j_{0}} \tau+\sqrt{1-\lambda_{j_{0}}^{2}}\left(\left(\max \kappa+\delta_{+}\right) \log |\tau|\right),+\infty\right) \\
\text { for } \delta_{-}, \delta_{+}>0
\end{gathered}
$$

(remark $\left.\min \kappa=-\left(k_{j_{0}}-1\right), \max \kappa=+\left(k_{j_{0}}-1\right)\right)$.

Geometrically, we have defined logarithmically increasing sectors, namely $S_{\kappa_{0}}=\left\{(\xi, \tau): \xi \in I_{\kappa_{0}}(\tau)\right\}$ containing the curve $\xi=\Gamma_{\kappa_{0}}(\tau)=$ $\lambda_{j_{0}} \tau+\sqrt{1-\lambda_{j_{0}}^{2}}\left(\kappa_{0} \log |\tau|\right), S_{\kappa_{1} \kappa_{2}}=\left\{(\xi, \tau): \xi \in I_{\kappa_{1} \kappa_{2}}(\tau)\right\}$ between two such curves, and $S_{-\infty}=\left\{(\xi, \tau): \xi \in I_{-\infty}(\tau)\right\}, S_{+\infty}=\{(\xi, \tau)$ : $\left.\xi \in I_{+\infty}(\tau)\right\}$ at the outside. Again our aim is to find estimates in each of the sectors showing that the only contributions to the cluster come from the participating soliton $u_{a_{j_{0}}, \kappa_{0}}^{+}$in the sector $S_{\kappa_{0}} \forall \kappa_{0}$. More precisely, $u_{a_{j_{0}}, \kappa_{0}}^{+}$dominates in the sector $S_{\kappa_{0}}$, and all other terms under consideration decay to zero. 
a) Now fix $\kappa_{0}$ and take $\xi \in I_{\kappa_{0}}(\tau)$, i.e.

$$
\xi=\Gamma_{\kappa_{0}+\rho}(\tau) \quad \text { with } \quad \rho \in\left(-\delta_{\kappa_{0}}, \delta_{\kappa_{0}}\right) .
$$

Note that we now have to replace $\rho$ in (1), (2) by $\rho+\kappa_{0}$.

In (6.32), the order of $\tau$ is determined by the exponents

$$
F_{\rho}(m)=-m^{2}+\left(k_{j_{0}}+\kappa_{0}+\rho\right) m, \quad m \in\left\{0, \ldots, k_{j_{0}}\right\} .
$$

Since $F_{\rho}$ has a global maximum in $m_{0}=\frac{k_{j_{0}}+\kappa_{0}+\rho}{2}=\left(\kappa_{0}^{\prime}+\frac{1}{2}\right)+\frac{\rho}{2} \in \mathbf{R}$ and $|\rho|<\frac{1}{2}$, the dominating exponents in (6.32) are attained for the two summands $m=\kappa_{0}^{\prime}, \kappa_{0}^{\prime}+1$. Moreover, the distance to the other exponents in the sum (6.32) is at least

$$
\begin{aligned}
\min \left\{F_{\rho}\left(\kappa_{0}^{\prime}\right),\right. & \left.\left.\left.F_{\rho}\left(\kappa_{0}^{\prime}+1\right)\right)\right\}-\max \left\{F_{\rho}\left(\kappa_{0}^{\prime}-1\right), F_{\rho}\left(\kappa_{0}^{\prime}+2\right)\right)\right\} \\
& =\left\{\begin{array}{ccc}
F_{\rho}\left(\kappa_{0}^{\prime}+1\right)-F_{\rho}\left(\kappa_{0}^{\prime}-1\right) & =2(1+\rho), \quad \rho \leq 0 \\
F_{\rho}\left(\kappa_{0}^{\prime}\right)-F_{\rho}\left(\kappa_{0}^{\prime}+2\right) & =2(1-\rho), \quad \rho \geq 0
\end{array}\right.
\end{aligned}
$$

Therefore we obtain in $(6.32)$

$$
\begin{aligned}
& p_{a_{j_{0}}}^{+}\left(\Gamma_{\kappa_{0}+\rho}(\tau), \tau\right)= \\
& =(-\mathrm{i})^{\sum_{j \in \Lambda_{j_{0}}^{+}} k_{j}} X^{+}(-\mathrm{i})^{\kappa^{\prime}}\left(\Omega_{\kappa_{0}^{\prime}}^{+} X_{\kappa_{0}^{\prime}}^{+}-\mathrm{i} \Omega_{\kappa_{0}^{\prime}+1}^{+} X_{\kappa_{0}^{\prime}+1}^{+} \tau^{\rho}\right) \tau^{\kappa_{0}^{\prime}\left(\kappa_{0}^{\prime}+1+\rho\right)} \cdot\left[1+\mathcal{O}\left(\frac{\log |\tau|}{\tau}\right)\right],
\end{aligned}
$$

and hence,

$$
\frac{p_{a_{j_{0}}}^{+}\left(\Gamma_{\kappa_{0}+\rho}(\tau), \tau\right)}{\overline{p_{a_{j_{0}}^{+}}^{+}}\left(\Gamma_{\kappa_{0}+\rho}(\tau), \tau\right)}=(-1)^{\kappa_{0}^{\prime}+\sum_{j \in \Lambda_{j_{0}}^{+}} k_{j}} \frac{1-\mathrm{i} \mathcal{X}_{\kappa_{0}^{\prime}}^{+} \tau^{\rho}}{1+\mathrm{i} \mathcal{X}_{\kappa_{0}^{\prime}}^{+} \tau^{\rho}}+\mathcal{O}\left(\frac{\log |\tau|}{\tau}\right),
$$

with $\mathcal{X}_{\kappa_{0}^{\prime}}^{+}=\frac{\Omega_{\kappa_{0}^{\prime}+1}^{+}}{\Omega_{\kappa_{0}^{\prime}}^{+}} \frac{X_{\kappa_{0}^{\prime}+1}^{+}}{X_{\kappa_{0}^{\prime}}^{+}}=(-1) \frac{\left(k_{j_{0}}-1\right)-\kappa_{0}}{2} \exp \left(\varphi_{j_{0}}+\varphi_{j_{0}}^{+}+\varphi_{j_{0}, \kappa_{0}}^{+}\right)$.

Since the latter expression in (6.39) equals $(-1)^{\kappa_{0}^{\prime}+\sum_{j \in \Lambda_{j_{0}}^{+}} k_{j}} \frac{p_{a_{j_{0}}, \kappa_{0}}^{+}\left(\Gamma_{\kappa_{0}+\rho}(\tau), \tau\right)}{\overline{p_{a_{0}, \kappa_{0}}^{+}}\left(\Gamma_{\kappa_{0}+\rho}(\tau), \tau\right)}$, we have proved that the cluster $\left(u_{a_{j_{0}}}^{+}+c_{j_{0}}^{+}\right)$converges to $\left(u_{a_{j_{0}, \kappa_{0}}^{+}}^{+}+2 \mathrm{i} \log (-1)^{\kappa_{0}^{\prime}+\sum_{j \in \Lambda_{j_{0}}^{+}} k_{j}}\right)$ uniformly in $S_{\kappa 0}$. 
In an analogous way to the modifications in (6.39), one shows

$$
\begin{aligned}
\frac{p_{a_{j_{0}, \kappa_{*}}}^{+}\left(\Gamma_{\kappa_{0}+\rho}(\tau), \tau\right)}{\overline{p_{a_{0}, \kappa_{*}}^{+}}\left(\Gamma_{\kappa_{0}+\rho}(\tau), \tau\right)} & =\frac{1-\mathrm{i} \mathcal{X}_{\kappa_{*}^{\prime}}^{+} \tau^{\kappa_{0}-\kappa_{*}+\rho}}{1+\mathrm{i} \mathcal{X}_{\kappa_{*}^{\prime}}^{+} \tau^{\kappa_{0}-\kappa_{*}+\rho}} \quad\left(\mathcal{X}_{\kappa_{*}^{\prime}}^{+} \text {defined as above }\right) \\
& =\left\{\begin{array}{cc}
1+\mathcal{O}\left(\frac{1}{\tau}\right), & \kappa_{0}<\kappa_{*} \\
-1+\mathcal{O}\left(\frac{1}{\tau}\right), & \kappa_{0}>\kappa_{*}
\end{array} .\right.
\end{aligned}
$$

Thus, $u_{a_{j_{0}}, \kappa_{*}}^{+}$converges to $\left\{\begin{array}{cl}0, & \text { for } \kappa_{0}<\kappa_{*} \\ 2 \mathrm{i} \log (-1) & \text { for } \kappa_{0}>\kappa_{*}\end{array}\right.$ uniformly in $S_{\kappa_{0}}$.

$$
\text { Remarking } c_{j_{0}}^{+}-2 \mathrm{i} \log (-1)^{\kappa_{0}^{\prime}+\sum_{j \in \Lambda_{j}^{+}} k_{j}}=-2 \mathrm{i} \log (-1)^{\kappa_{0}^{\prime}}=
$$

$-2 \mathrm{i} \log (-1)^{\#\left\{\kappa: \kappa<\kappa_{0}\right\}}$, we infer the desired convergence of $u_{a_{j_{0}}}^{+}$(uniformly in $S_{\kappa_{0}}$ ) from

$$
\begin{array}{r}
u_{a_{j_{0}}}^{+}-\sum_{\kappa} u_{a_{j_{0}, \kappa}}^{+}=\left(\left(u_{a_{j_{0}}}^{+}+c_{j_{0}}^{+}\right)-\left(u_{a_{j_{0}}, \kappa_{0}}^{+}+2 \mathrm{i} \log (-1)^{\kappa_{0}^{\prime}+\sum_{j \in \Lambda_{j_{0}}^{+}} k_{j}}\right)\right) \\
-\sum_{\kappa_{0}<\kappa} u_{a_{j_{0}, \kappa}^{+}}^{+}-\left(\sum_{\kappa<\kappa_{0}} u_{a_{j_{0}}, \kappa}^{+}-2 \mathrm{i} \log (-1)^{\kappa_{0}^{\prime}}\right) .
\end{array}
$$

b) Next, fix $\kappa_{1}<\kappa_{2}$ such that there is no $\kappa \in\left(\kappa_{1}, \kappa_{2}\right)$ and take $\xi \in$ $I_{\kappa_{1} \kappa_{2}}(\tau)$, i.e.

$$
\xi=\Gamma_{\rho}(\tau) \quad \text { with } \quad \rho \in\left(\kappa_{1}+\delta_{\kappa_{1} \kappa_{2}}, \kappa_{2}-\delta_{\kappa_{1} \kappa_{2}}\right) .
$$

Again we determine the maximal order of $\tau$ in (6.32). Since $\widetilde{F}_{\rho}(m)=$ $-m^{2}+\left(k_{j_{0}}+\rho\right) m$ attains a global maximum in $m_{0}=\frac{k_{j_{0}}+\rho}{2} \in\left(\kappa_{1}^{\prime}+\right.$ $\left.\frac{1+\delta_{\kappa_{1} \kappa_{2}}}{2}, \kappa_{2}^{\prime}+\frac{1-\delta_{\kappa_{1} \kappa_{2}}}{2}\right)$ for $\rho \in\left(\kappa_{1}+\delta_{\kappa_{1} \kappa_{2}}, \kappa_{2}-\delta_{\kappa_{1} \kappa_{2}}\right)$, the dominating summand in (6.32) is $m=\kappa_{1}^{\prime}+1$. Once more we calculate the distance to the other exponents, at least we get

$$
\begin{array}{ll}
\widetilde{F}_{\rho}\left(\kappa_{1}^{\prime}+1\right)-\widetilde{F}_{\rho}\left(\kappa_{1}^{\prime}\right) & =\rho-\kappa_{1} \\
\widetilde{F}_{\rho}\left(\kappa_{1}^{\prime}+1\right)-\widetilde{F}_{\rho}\left(\kappa_{1}^{\prime}+2\right) & =\kappa_{2}-\rho
\end{array} \quad>\delta_{\kappa_{1} \kappa_{2}}>0
$$

This shows

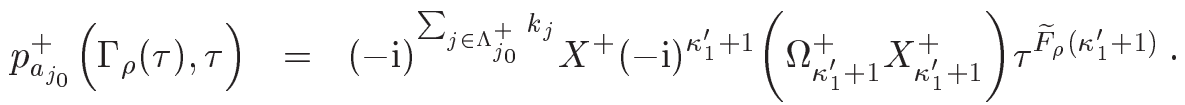

$$
\begin{aligned}
& \cdot\left[1+\mathcal{O}\left(\frac{\log |\tau|}{\tau}\right)+\mathcal{O}\left(\frac{1}{\tau^{\delta_{\kappa_{1} \kappa_{2}}}}\right)\right]
\end{aligned}
$$


where $\widetilde{F}_{\rho}\left(\kappa_{1}^{\prime}+1\right)>\kappa_{1}^{\prime}+\delta_{\kappa_{1} \kappa_{2}}>0$, and, consequently,

$$
\frac{p_{a_{j_{0}}}^{+}\left(\Gamma_{\rho}(\tau), \tau\right)}{\overline{p_{a_{j_{0}}}^{+}}\left(\Gamma_{\rho}(\tau), \tau\right)}=(-1)^{\kappa_{1}^{\prime}+1+\sum_{j \in \Lambda_{j_{0}}^{+}} k_{j}}+\mathcal{O}\left(\frac{1}{\tau^{\delta_{\kappa_{1} \kappa_{2}}}}\right) .
$$

Thus, we have proved that $\left(u_{a_{0}}^{+}+c_{j_{0}}^{+}\right)$converges to the constant $2 \mathrm{i} \log (-1)^{\#\left\{\kappa: \kappa \leq \kappa_{1}\right\}+\sum_{j \in \Lambda_{j_{0}}^{+}} k_{j}}$ uniformly in the sector $S_{\kappa_{1} \kappa_{2}}$.

Just as in a), one shows easily

$$
\frac{p_{a_{j_{0}}, \kappa_{*}}^{+}\left(\Gamma_{\rho}(\tau), \tau\right)}{\overline{p_{a_{j_{0}}, \kappa_{*}}^{+}}\left(\Gamma_{\rho}(\tau), \tau\right)}=\left\{\begin{array}{cl}
1+\mathcal{O}\left(\frac{1}{\tau^{\delta_{\kappa_{1} \kappa_{2}}}}\right), & \kappa_{2} \leq \kappa_{*} \\
-1+\mathcal{O}\left(\frac{1}{\tau^{\delta_{\kappa_{1} \kappa_{2}}}}\right), & \kappa_{*} \leq \kappa_{1}
\end{array},\right.
$$

that is, $u_{a_{j_{0}}, \kappa_{*}}^{+}$converges to $\left\{\begin{array}{cc}0, & \text { for } \kappa_{2} \leq \kappa_{*} \\ 2 \mathrm{i} \log (-1) & \text { for } \kappa_{*} \leq \kappa_{1}\end{array}\right.$ uniformly in $S_{\kappa_{1} \kappa_{2}}$.

The convergence of $u_{a_{j_{0}}}^{+}$(uniformly in $S_{\kappa_{1} \kappa_{2}}$ ) now follows from

$$
\begin{array}{r}
u_{a_{j_{0}}}^{+}-\sum_{\kappa} u_{a_{j_{0}}, \kappa}^{+}=\left(\left(u_{a_{j_{0}}}^{+}+c_{j_{0}}^{+}\right)-2 \mathrm{i} \log (-1)^{\#\left\{\kappa: \kappa \leq \kappa_{1}\right\}+\sum_{j \in \Lambda_{j_{0}}^{+}} k_{j}}\right) \\
-\sum_{\kappa_{2} \leq \kappa} u_{a_{j_{0}}, \kappa}^{+}-\left(\sum_{\kappa \leq \kappa_{1}} u_{a_{j_{0}, \kappa}}^{+}-2 \mathrm{i} \log (-1)^{\#\left\{\kappa: \kappa \leq \kappa_{1}\right\}}\right)
\end{array}
$$

in the usual manner.

c) Next consider $\xi \in I_{-\infty}(\tau)$ or $\xi \in I_{+\infty}(\tau)$, i.e.

$$
\begin{aligned}
& \xi=\Gamma_{\rho}(\tau) \text { with } \rho \in\left(-\infty,-\left(k_{j_{0}}-1\right)-\delta_{-}\right) \quad \text { or } \\
& \xi=\Gamma_{\rho}(\tau) \text { with } \rho \in\left(\left(k_{j_{0}}-1\right)+\delta_{+},+\infty\right) .
\end{aligned}
$$


Repeating the arguments of $\mathbf{b}$ ), we obtain

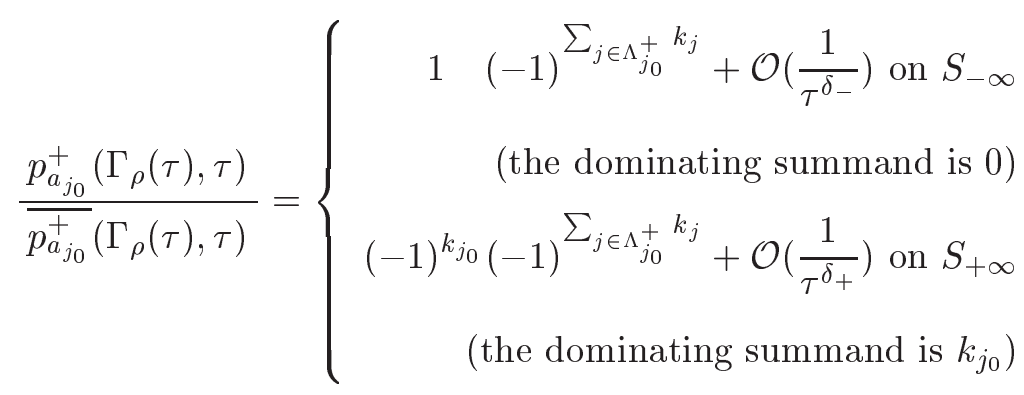

and

$$
\frac{p_{a_{j_{0}, \kappa_{*}}}^{+}\left(\Gamma_{\rho}(\tau), \tau\right)}{\overline{p_{a_{j_{0}}, \kappa_{*}}^{+}}\left(\Gamma_{\rho}(\tau), \tau\right)}=\left\{\begin{array}{ccc}
1+\mathcal{O}\left(\frac{1}{\tau^{\delta_{-}}}\right) & \forall \kappa_{*} & \text { on } S_{-\infty} \\
-1+\mathcal{O}\left(\frac{1}{\tau^{\delta_{+}}}\right) & \forall \kappa_{*} & \text { on } S_{+\infty} .
\end{array}\right.
$$

The same reasoning as in b) shows that $u_{a_{j_{0}}}^{+}$converges to $\sum_{\kappa} u_{a_{j_{0}, \kappa}}^{+}$, uniformly in $S_{-\infty}$ and $S_{+\infty}$.

d) Finally, we remark that all constants $\delta_{\kappa_{0}}, \delta_{\kappa_{1} \kappa_{2}}$ and $\delta_{+}, \delta_{-}$can be chosen such that the intervals $I_{\kappa_{0}}(\tau)\left(\forall \kappa_{0}\right), I_{\kappa_{1} \kappa_{2}}(\tau)\left(\forall \kappa_{1}, \kappa_{2}\right.$ possible) and $I_{+\infty}(\tau), I_{-\infty}(\tau)$ cover $\mathbf{R}(\forall \tau)$.

This immediately proves that the desired convergence assertion holds even uniformly in $\mathbf{R}$.

(4) At last, we indicate the notational changes in the treatment of clusters corresponding to a pair of complex conjugate eigenvalues $a_{\bar{\jmath}_{0}}=\bar{a}_{j_{0}}$.

For a pair of complex conjugated eigenvalues, $a_{\bar{\jmath}_{0}}=\bar{a}_{j_{0}}$ with $\Im\left(a_{j_{0}}\right) \geq$ 0 , we used the notation $a_{j_{0}}=\left|a_{j_{0}}\right|\left(\sqrt{1-\omega_{j_{0}}^{2}}+\mathrm{i} \omega_{j_{0}}\right), a_{\bar{\jmath}_{0}}=\left|a_{j_{0}}\right|\left(\sqrt{1-\omega_{j_{0}}^{2}}-\right.$ $\left.\mathrm{i} \omega_{j_{0}}\right)$.

Here, the logarithmic curves $\xi=\Gamma_{\rho}(\tau)$ are defined in a slightly different manner,

$$
\xi=\Gamma_{\rho}(\tau) \Longleftrightarrow \sqrt{1-\omega_{j_{0}}^{2}} \frac{\xi-\lambda_{j_{0}} \tau}{\sqrt{1-\lambda_{j_{0}}^{2}}}=\rho \log |\tau| .
$$

On this curve, one proves the estimate

$\ell_{j}^{(\kappa)}\left(\Gamma_{\rho}(\tau), \tau\right)=\frac{\left(-d_{j}\right)^{\kappa}}{\kappa !} \exp \left(\phi_{j}(\tau)\right) \tau^{\kappa}|\tau|^{\rho} \cdot\left[1+\mathcal{O}\left(\frac{\log |\tau|}{\tau}\right)\right] \quad \forall \kappa$ 
for $j \in\left\{j_{0}, \bar{\jmath}_{0}\right\}$,

where $d_{j_{0}}=\frac{2 \Re\left(a_{j_{0}}\right)}{a_{j_{0}}\left(1+\left|a_{j_{0}}\right|^{2}\right)}, \phi_{j_{0}}(\tau)=-\mathrm{i} \omega_{j_{0}} \frac{\tau-\lambda_{j_{0}} \Gamma_{\rho}(\tau)}{\sqrt{1-\lambda_{j_{0}}^{2}}}+f_{j_{0}}\left(a_{j_{0}}\right), d_{\bar{\jmath}_{0}}=$ $\bar{d}_{j_{0}}$, and $\phi_{\bar{j}_{0}}(\tau)=\bar{\phi}_{j_{0}}(\tau)$.

For the investigation of the determinant $p_{a_{j_{0}}}^{+}=\operatorname{det}\left(V_{j j^{\prime}}\right)_{j, j^{\prime} \in \Lambda_{j_{0}}^{+} \cup\left\{j_{0}, \bar{J}_{0}\right\}}$ on the logarithmic curve, we have to generalize the notations introduced in (2) in the following manner:

$$
\text { Let } T=\left(T_{j j^{\prime}}\right)_{j j^{\prime}} \text { with } T_{j j^{\prime}}=\left(T_{j j^{\prime}}^{\left(\kappa \kappa^{\prime}\right)}\right)_{\substack{\kappa=1, \ldots, k_{j} \\ \kappa^{\prime}=1, \ldots, k_{j^{\prime}}}} \text {. For index sets }
$$

$J, \bar{J} \subseteq\left\{1, \ldots, k_{j_{0}}\right\}$, we denote by $T[J, \bar{J}]$ the matrix built from $T$ restricting the index sets by

$$
\begin{array}{ll}
\kappa \in J & \text { if } j=j_{0} \\
\kappa \in \bar{J} & \text { if } j=\bar{\jmath}_{0}
\end{array} \quad \text { and } \quad \begin{array}{ll}
\kappa^{\prime} \in J & \text { if } j^{\prime}=j_{0} \\
\kappa^{\prime} \in \bar{J} & \text { if } j^{\prime}=\bar{\jmath}_{0}
\end{array} .
$$

With this notations, the expansion of the determinant results in $\operatorname{det}(V)=$ $\operatorname{det}\left(\widehat{V}_{j j^{\prime}}\right)_{j, j^{\prime} \in \Lambda_{j_{0}}^{+}}+\sum_{m=1}^{k_{j_{0}}} \sum_{\bar{m}=1}^{k_{j_{0}}} \sum_{\substack{\sigma_{1}<\ldots<\sigma_{m} \\ \bar{\sigma}_{1}<\ldots<\overline{\sigma_{m}}}} \operatorname{det}\left(\widehat{V}\left[\left\{\sigma_{1}, \ldots, \sigma_{m}\right\},\left\{\bar{\sigma}_{1}, \ldots, \bar{\sigma}_{\bar{m}}\right\}\right]\right)$ for $V=\left(V_{j j^{\prime}}\right)_{j, j^{\prime} \in \Lambda_{j_{0}}^{+} \cup\left\{j_{0}, \bar{\jmath}_{0}\right\}}, \widehat{V}=\left(\widehat{V}_{j j^{\prime}}\right)_{j, j^{\prime} \in \Lambda_{j_{0}}^{+} \cup\left\{j_{0}, \bar{\jmath}_{0}\right\}}$, and, analogously to $(2)$,

$$
\begin{gathered}
\operatorname{det}\left(\widehat{V}\left[\left\{\sigma_{1}, \ldots, \sigma_{m}\right\},\left\{\bar{\sigma}_{1}, \ldots, \bar{\sigma}_{\bar{m}}\right\}\right]\right)= \\
=\sum_{\sigma_{1}^{\prime}=1}^{k_{j_{0}}-\sigma_{1}+1} \ldots \sum_{\sigma_{m}^{\prime}=1}^{k_{j_{0}}-\sigma_{m}+1} \ell_{j_{0}}^{\left(k_{j_{0}}+1-\sigma_{1}-\sigma_{1}^{\prime}\right)} \ldots \ell_{j_{0}}^{\left(k_{j_{0}}+1-\sigma_{m}-\sigma_{m}^{\prime}\right)} . \\
\sum_{\bar{\sigma}_{1}^{\prime}=1}^{k_{j_{0}}-\bar{\sigma}_{1}+1} \cdots \sum_{\bar{\sigma}_{\bar{m}}^{\prime}=1}^{k_{j_{0}}-\bar{\sigma}_{\bar{m}}+1} \ell_{j_{0}}^{\left(k_{j_{0}}+1-\bar{\sigma}_{1}-\bar{\sigma}_{1}^{\prime}\right)} \ldots \ell_{j_{0}}^{\left(k_{j_{0}}+1-\bar{\sigma}_{\bar{m}}-\bar{\sigma}_{\bar{m}}^{\prime}\right)} . \\
\operatorname{det}\left(-\mathrm{i} W\left[\left\{\sigma_{1}^{\prime}, \ldots, \sigma_{m}^{\prime}\right\},\left\{\bar{\sigma}_{1}^{\prime}, \ldots, \bar{\sigma}_{\bar{m}}^{\prime}\right\}\right]\right), \\
\text { where }-\mathrm{i} W=\left(-\mathrm{i} W_{j j^{\prime}}\right)_{j, j^{\prime} \in \Lambda_{j_{0}}^{+} \cup\left\{j_{0}, \bar{J}_{0}\right\}} .
\end{gathered}
$$


Now one may follow precisely the line of reasoning in (2) with the only difference that there are always two expressions to bear in mind instead of one. Those expressions, one in $m$, one in $\bar{m}$, are of the same kind and can be treated separately. We end up with

$$
\begin{array}{r}
p_{a_{j_{0}}}^{+}\left(\Gamma_{\rho}(\tau), \tau\right)=(-\mathrm{i})^{\sum_{j \in \Lambda_{j_{0}}^{+}}^{k_{j}} X^{+}\left(1+\sum_{m=1}^{k_{j_{0}}} \sum_{\bar{m}=1}^{k_{j_{0}}}(-\mathrm{i})^{m+\bar{m}} \Omega_{m, \bar{m}}^{+} X_{m}^{+} \bar{X}_{\bar{m}}^{+} .\right.} \\
\left.|\tau|^{\rho(m+\bar{m})}(\tau)^{\left(k_{j_{0}}-m\right) m+\left(k_{j_{0}}-\bar{m}\right) \bar{m}}\left[1+\mathcal{O}\left(\frac{\log |\tau|}{\tau}\right)\right]\right)
\end{array}
$$

with $X^{+}=\operatorname{det}\left(W_{j j^{\prime}}\right)_{j, j^{\prime} \in \Lambda_{j_{0}}^{+}}$,

$X_{m}^{+}=(-1)^{\frac{m(m+3)}{2}} \frac{\prod_{\kappa=1}^{m-1} \kappa !}{\prod_{\kappa=1}^{m}\left(k_{j_{0}}-\kappa\right) !}\left(\left(-d_{j_{0}}\right)^{k_{j_{0}}-m} \exp \left(\phi_{j_{0}}(\tau)+f_{j_{0}}\left(a_{j_{0}}\right)\right)\right)^{m}$,

$\bar{X}_{\bar{m}}^{+}=(-1)^{\frac{\bar{m}(\bar{m}+3)}{2}} \frac{\prod_{\kappa=1}^{\bar{m}-1} \kappa !}{\prod_{\kappa=1}^{\bar{m}}\left(k_{j_{0}}-\kappa\right) !}\left(\left(-\bar{d}_{j_{0}}\right)^{k_{j_{0}}-\bar{m}} \exp \left(\bar{\phi}_{j_{0}}(\tau)+\overline{f_{j_{0}}\left(a_{j_{0}}\right)}\right)\right)^{\bar{m}}$,

and

$\Omega_{m, \bar{m}}^{+}=\left[\frac{1}{2 a_{j_{0}}}\right]^{m^{2}}\left[\frac{1}{2 \bar{a}_{j_{0}}}\right]^{\bar{m}^{2}}\left[\frac{a_{j_{0}}-\bar{a}_{j_{0}}}{a_{j_{0}}+\bar{a}_{j_{0}}}\right]^{2 m \bar{m}} \prod_{j \in \Lambda_{j_{0}}^{+}}\left(\left[\frac{a_{j}-a_{j_{0}}}{a_{j}+a_{j_{0}}}\right]^{m}\left[\frac{a_{j}-\bar{a}_{j_{0}}}{a_{j}+\bar{a}_{j_{0}}}\right]^{\bar{m}}\right)^{2 k_{j}}$,

and we remark

$$
\left[\frac{a_{j_{0}}-\bar{a}_{j_{0}}}{a_{j_{0}}+\bar{a}_{j_{0}}}\right]^{2 m \bar{m}}=\left(-\alpha_{j_{0}}^{2}\right)^{m \bar{m}} \quad \text { for } \quad \alpha_{j_{0}}=\frac{\omega_{j_{0}}}{\sqrt{1-\omega_{j_{0}}^{2}}}
$$

At this point, it is quite clear that one can take over the argumentation in (3) with minor changes due to the fact that the power of $\tau$ has to be maximized for $m, \bar{m}$ separately. Thus, here we omit the lengthy analysis to show uniform convergence on the logarithmic sectors introduced in (3) just stating the result instead.

Result.

$$
u_{a_{j_{0}}}^{+}(\xi, \tau) \approx \sum_{\kappa_{0}} u_{a_{j_{0}}, \kappa_{0}}^{+}(\xi, \tau)
$$

with $\kappa_{0}=-\left(k_{j_{0}}-1\right)+2 \kappa_{0}^{\prime}$ for $\kappa_{0}^{\prime}=0, \ldots, k_{j_{0}}-1$ (vice versa, $\kappa_{0}^{\prime}=$ $\left.\frac{\left(k_{j_{0}}-1\right)+\kappa_{0}}{2}\right)$. The participating pulsating solitons $u_{a_{j_{0}}, \kappa_{0}}^{+}(\xi, \tau)$ are defined along the curves

$$
\mathcal{A}_{a_{j_{0}, \kappa_{0}}}(\xi, \tau)=\sqrt{1-\omega_{j_{0}}^{2}} \frac{\xi-\lambda_{j_{0}} \tau}{\sqrt{1-\lambda_{j_{0}}^{2}}}+\mathrm{i} \psi_{j_{0}}(\xi, \tau)-\kappa_{0} \log |\tau|+
$$




$$
+\left(\varphi_{j_{0}}+\varphi_{j_{0}}^{+}+\varphi_{j_{0}, \kappa_{0}}^{+}\right)+\left(k_{j_{0}}+\kappa_{0}\right) \log \alpha_{j_{0}}
$$

by $\quad u_{a_{j_{0}, \kappa_{0}}}^{+}=2 \mathrm{i} \log \frac{p_{a_{j_{0}, \kappa_{0}}^{+}}^{+}}{\overline{p_{a_{j_{0}}, \kappa_{0}}^{+}}}$

with $p_{a_{j_{0}}, \kappa_{0}}^{+}(\xi, \tau)=1-\mathrm{i}(-1)^{\left(k_{j_{0}}-1\right)} \frac{1}{\alpha_{j_{0}}} \frac{2 \Re\left(\exp \left(\mathcal{A}_{a_{j_{0}, \kappa_{0}}}(\xi, \tau)\right)\right)}{1+\left|\exp \left(\mathcal{A}_{a_{j_{0}, \kappa_{0}}}(\xi, \tau)\right)\right|^{2}}$.

The constants are defined quite similar as before, namely

$$
\begin{gathered}
e^{\varphi_{j_{0}}}=\frac{\exp \left(f_{j_{0}}\left(a_{j_{0}}\right)\right)}{\left(2 a_{j_{0}}\right)^{k_{j_{0}}}}, \quad e^{\varphi_{j_{0}}^{+}}=\prod_{j \in \Lambda_{j_{0}}^{+}}\left(\frac{a_{j}-a_{j_{0}}}{a_{j}+a_{j_{0}}}\right)^{2 k_{j}}, \\
e^{\varphi_{j_{0}, \kappa_{0}}^{+}}=\frac{\left(\frac{\left(k_{j_{0}}-1\right)+\kappa_{0}}{2}\right) !}{\left(\frac{\left(k_{j_{0}}-1\right)-\kappa_{0}}{2}\right) !}\left(\frac{4 \Re\left(a_{j_{0}}\right)}{1+\left|a_{j_{0}}\right|^{2}}\right)^{-\kappa_{0}},
\end{gathered}
$$

and there is an oscillating term due to $\psi_{j_{0}}(\xi, \tau)=-\omega_{j_{0}} \frac{\tau-\lambda_{j_{0}} \xi}{\sqrt{1-\lambda_{j_{0}}^{2}}}$.

Step 3. To conclude Theorem C, we combine the results of Step 1 and Step 2, modify the formulas according to $2 \mathrm{i} \log \frac{1-\mathrm{i} z}{1+\mathrm{i} z}=4 \arctan (z)$, and adapt the notation of Theorem $\mathrm{C}$ for the two characteristic features of eigenvalues.

The reader should observe that at this point the ambiguities related to the choice of log disappear, and we can always work with the main branch of arctan.

\section{Computer graphics for the lowest dimensional cases}

In the final section, we assemble some computer graphics which we found instructive during the preparation. The main point is of course the difference between straight lines of solitary waves and logarithmic rayes of the members of clusters. Further the diagrams underline that the convergence we established by asymptotic formulas is in fact very rapid 
and that during the elastic collisions the shape is as well conserved as in the familiar case of solitons.

First we present pictures of the building blocks, solitons and breathers, and their derivatives, which will most often be more convenient for the visualization. Note that the derivative of the breather confirms nicely the interpretation of Novikov [18] explaining the breather as a bound state of a soliton and an antisoliton.

In the pictures, the variables $\xi$ and $\tau$ are depicted in canonical fashion. If not remarked differently, we always draw the derivatives.

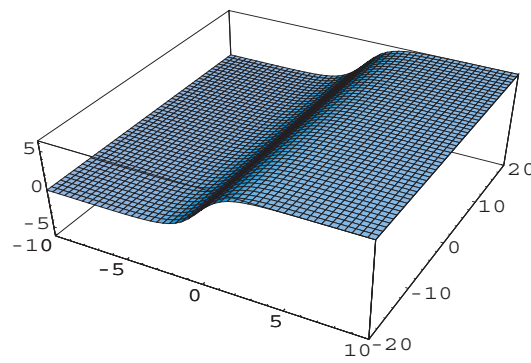

$\operatorname{kink}(b=1$, i.e. stationary)

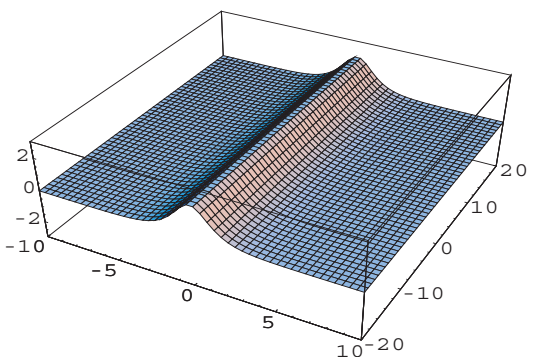

derivative of the kink

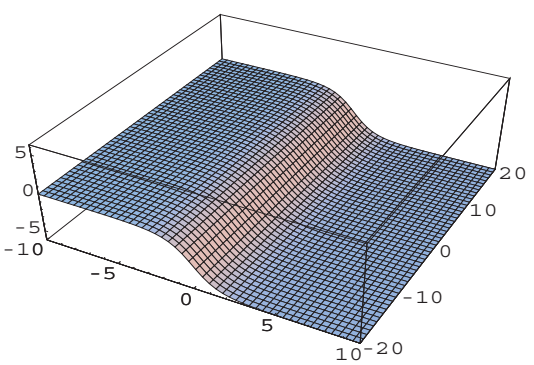

$\operatorname{antikink}(b=1$, i.e. stationary)

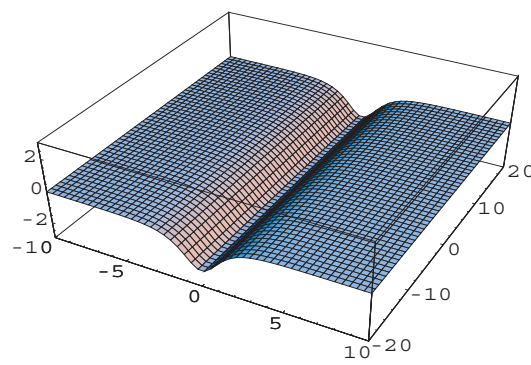

derivative of the antikink 

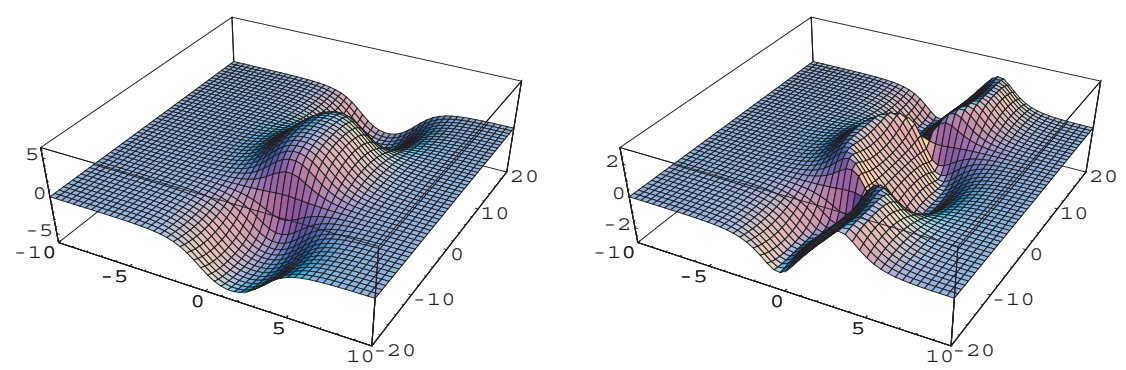

breather $\left(c=\sqrt{1-0.2^{2}}+0.2\right.$ i, i.e. stationary $)$ and its derivative

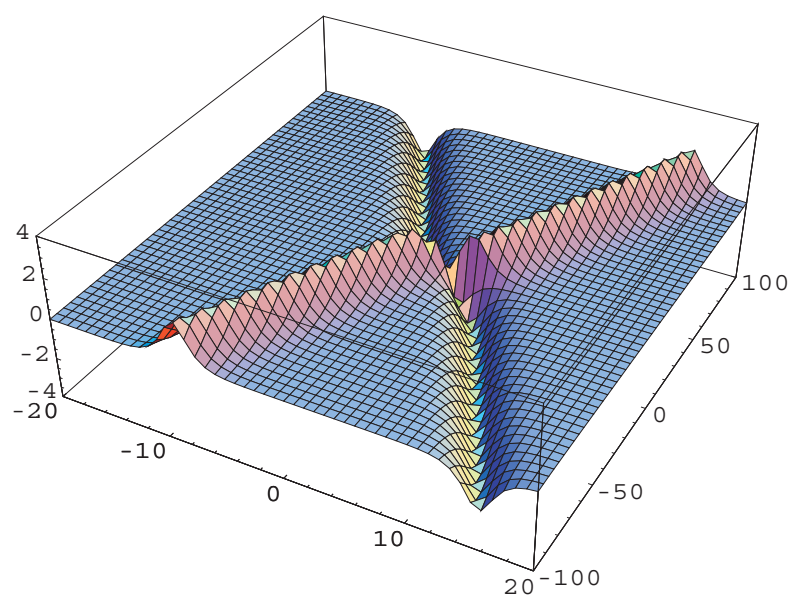

kink $\left(b_{1}=1.1\right)$ meets antikink $\left(b_{2}=0.9\right)$ 
C. SCHIEBOLD

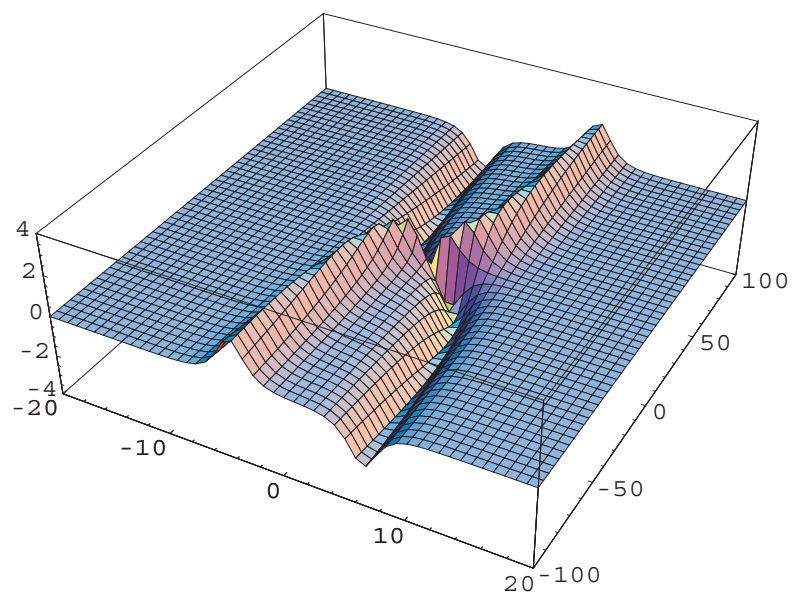

cluster $(b=1.0)$ of a kink and an antikink

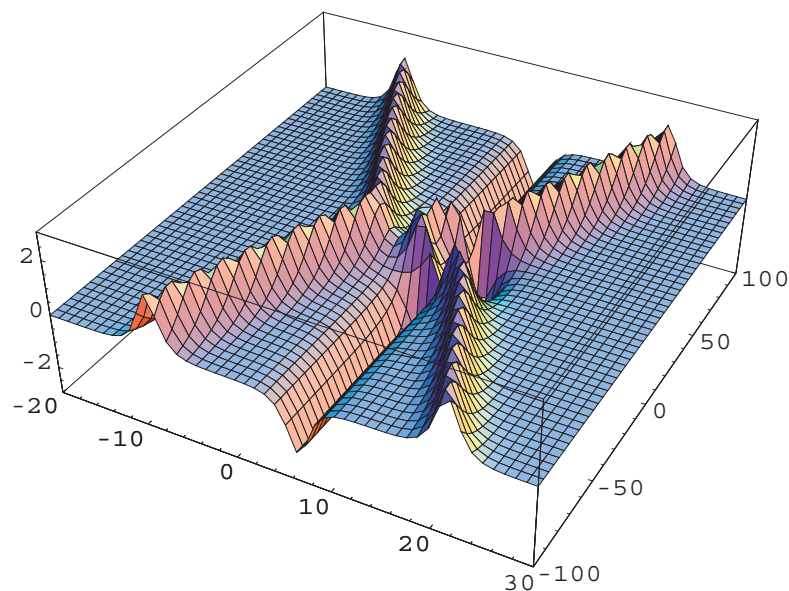

two kinks $\left(b_{1}=0.9, b_{2}=1.1\right)$ meet an antikink $\left(b_{3}=1.0\right)$ 
C. SCHIEBOLD SOLITONS OF THE SINE-GORDON EQUATION ...

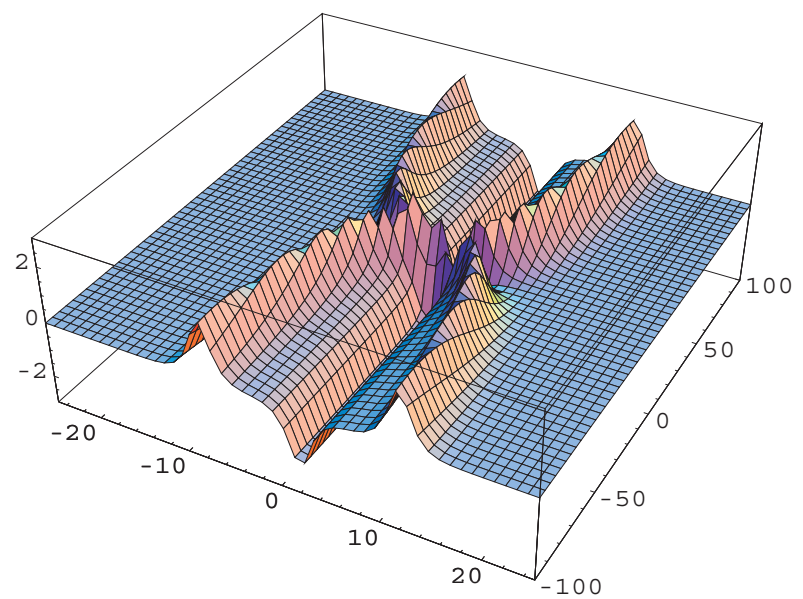

cluster $(b=1.0)$ of two kinks and an antikink

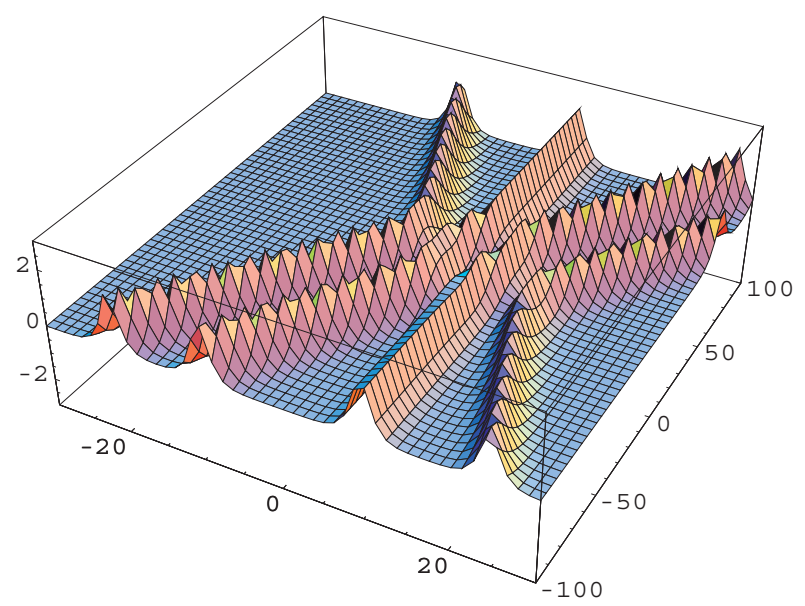

four kinks meet $\left(b_{1}=0.9, b_{2}=1.0, b_{3}=1.1\right.$, and $\left.b_{4}=1.25\right)$ 
C. SCHIEBOLD

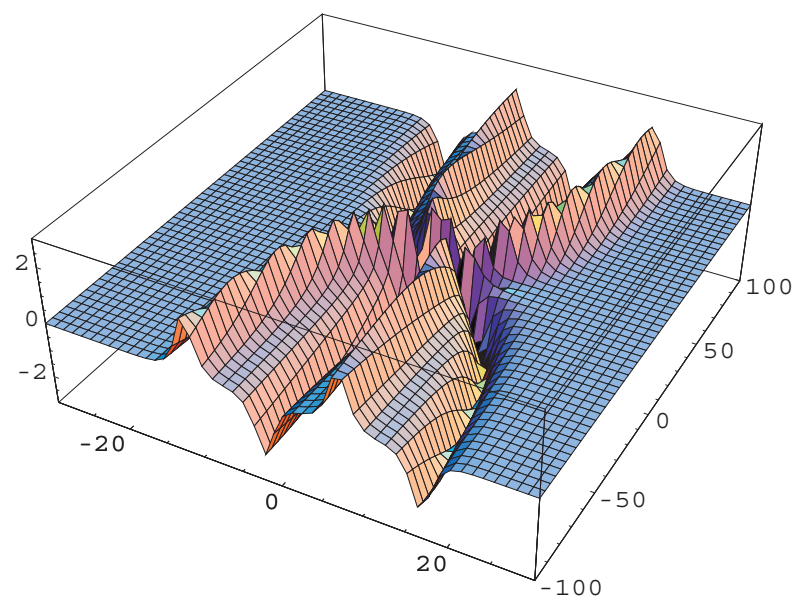

cluster $(b=1.0)$ of two kinks and two antikinks

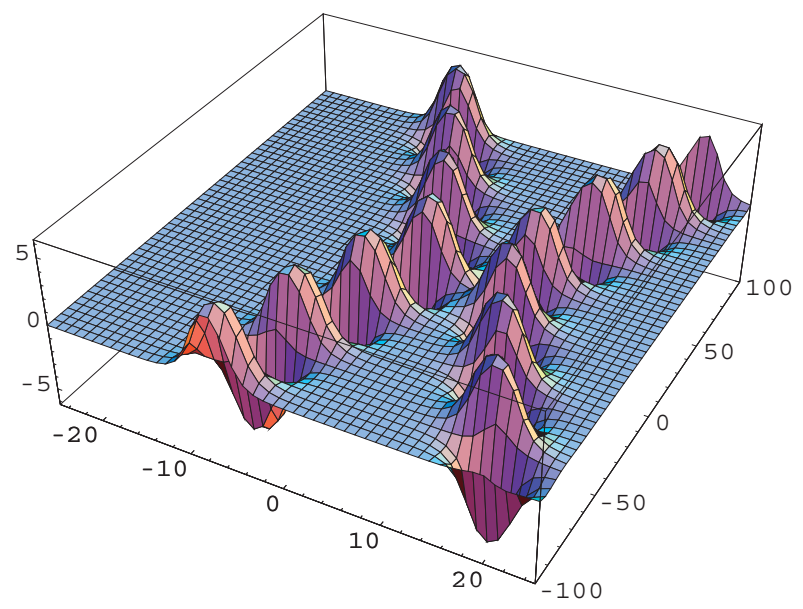

two breathers meet $\left(c_{1}=0.9\left(\sqrt{1-0.2^{2}}+0.2 \mathrm{i}\right), c_{2}=1.1\left(\sqrt{1-0.2^{2}}+0.2 \mathrm{i}\right)\right)$ (here we did not plot the derivative!) 
C. SCHIEBOLD SOLITONS OF THE SINE-GORDON EQUATION ...

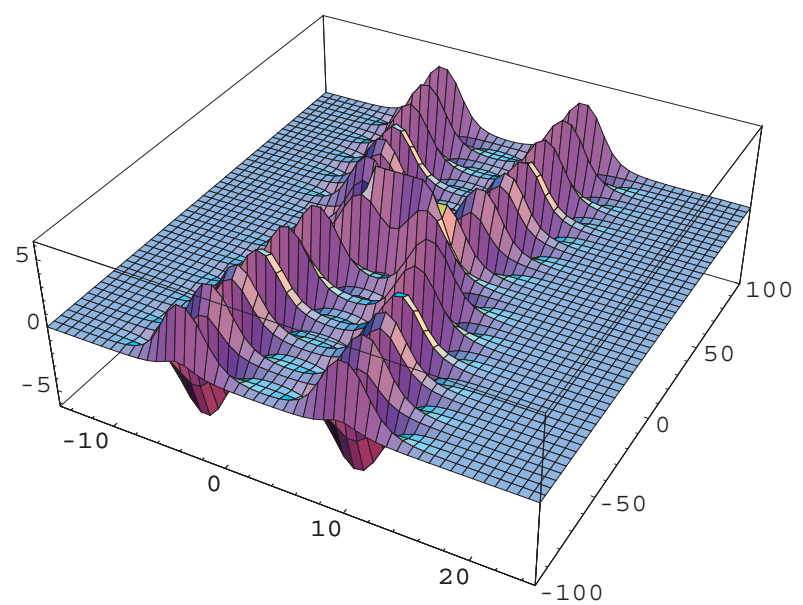

cluster $\left(c=\sqrt{1-0.4^{2}}+0.4 \mathrm{i}\right)$ of two breathers (here we did not plot the derivative!)

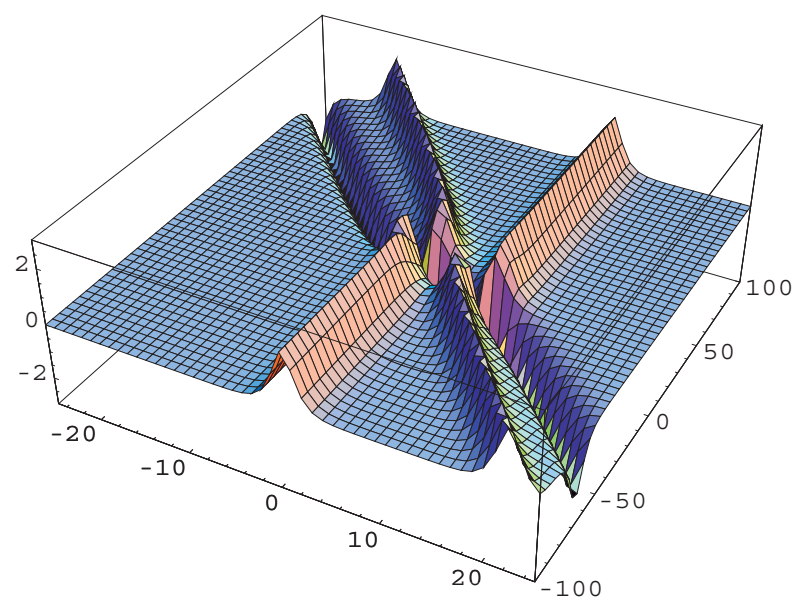

kink $\left(b_{1}=1.0\right)$ meets cluster $\left(b_{2}=0.8\right)$ of a kink and an antikink 
C. SCHIEBOLD

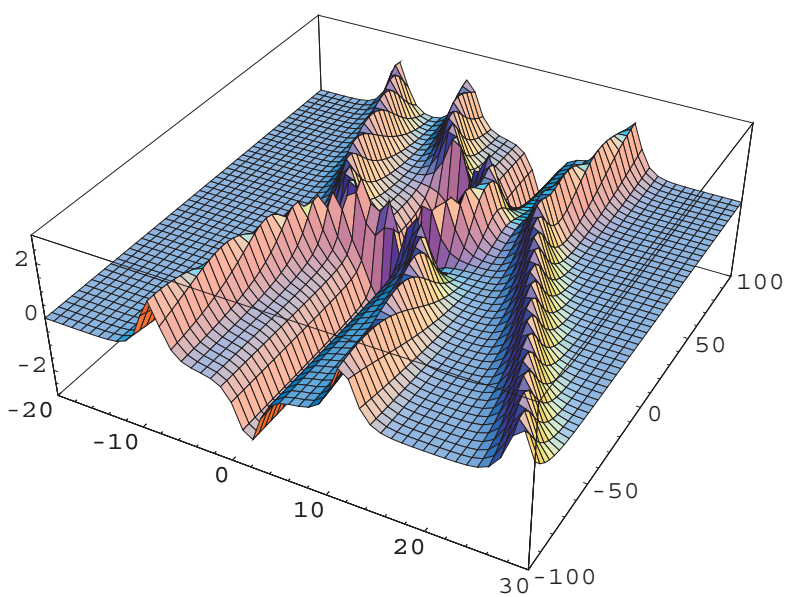

kink $\left(b_{1}=0.9\right)$ meets cluster $\left(b_{2}=1.0\right)$ of two kinks and an antikink

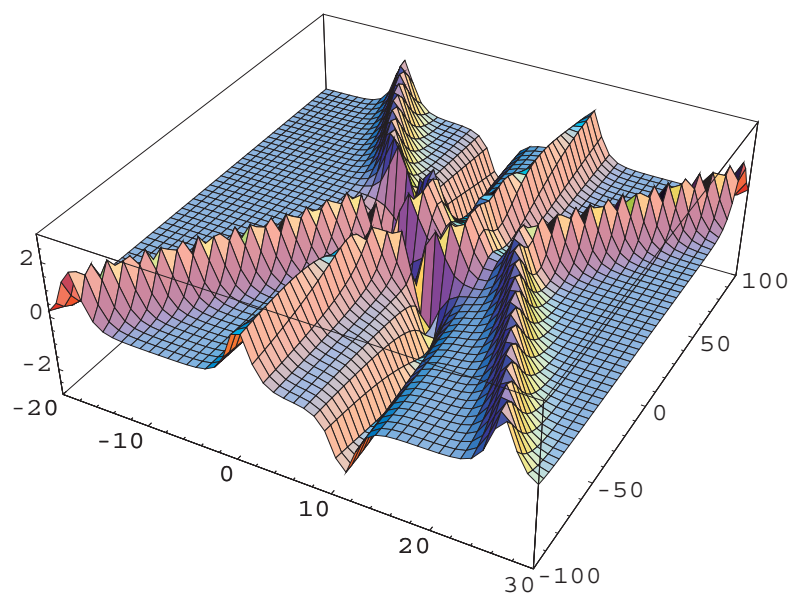

two kinks $\left(b_{1}=0.9, b_{2}=1.2\right)$ meet a cluster $\left(b_{3}=1.0\right)$ of a kink and an antikink 
C. SCHIEBOLD SOLITONS OF THE SINE-GORDON EQUATION ...

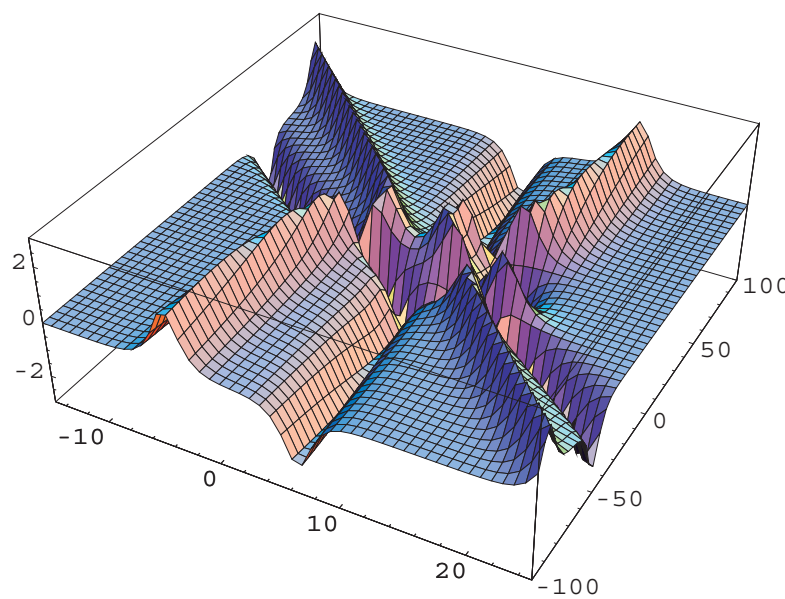

two clusters $\left(b_{1}=0.8, b_{2}=1.0\right)$ of a kink and an antikink meet

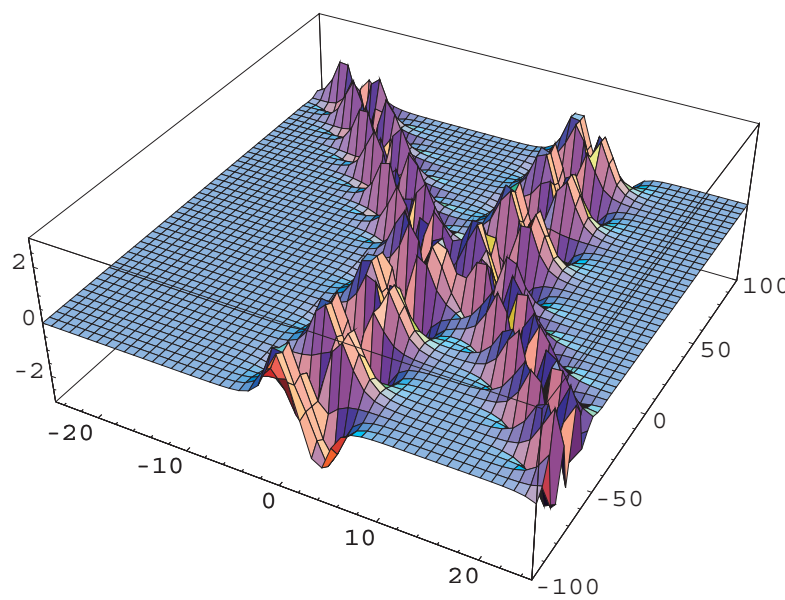

two breathers meet $\left(c_{1}=0.8\left(\sqrt{1-0.4^{2}}+0.4 \mathrm{i}\right), c_{2}=1.0\left(\sqrt{1-0.2^{2}}+0.2 \mathrm{i}\right)\right)$ 


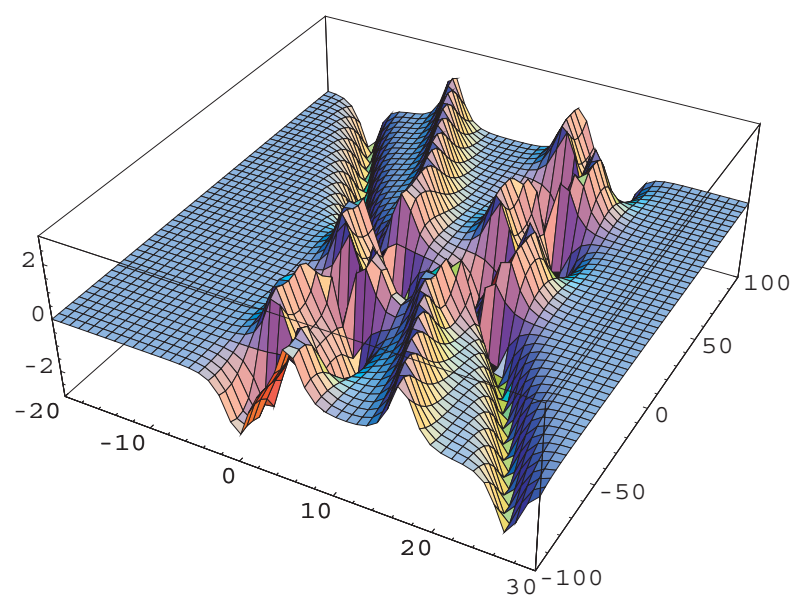

breather $\left(c=\sqrt{1-0.1^{2}}+0.1 \mathrm{i}\right)$ meets cluster $(b=0.9)$ of a kink and an antikink

\section{References}

[1] H. Aden and B. Carl, "On realizations of solutions of the KdV equation by determinants on operator ideals", J. Math. Phys. 37, 1833-1857 (1996).

[2] V. A. Andreev, "Matrix sine-Gordon equation", Theor. Math. Phys. 84, 920-929 (1990).

[3] R. Beutler, "Positon solutions of the sine-Gordon equation", J. Math. Phys. 34, 3098-3109 (1993).

[4] R. Bhatia and P. Rosenthal, "How and why to solve the operator equation $A X-X B=Y$ ", Bull. London Math. Soc. 29, 1-21 (1997).

[5] F. Calogero and A. Degasperis, "Nonlinear evolution equations solvable by the inverse spectal transform I", Nuovo Cimento 32B, 201242 (1976). 
[6] B. Carl and C. Schiebold, "Nonlinear equations in soliton physics and operator ideals", Nonlinearity 12, 333-364 (1999).

[7] F. Gesztesy, W. Karwowski, and Z. Zhao, "Limits of soliton solutions", Duke Math. J. 68, 101-150 (1992).

[8] F. Gesztesy and W. Renger, "New classes of Toda soliton solutions", Commun. Math. Phys. 184, 27-50 (1997).

[9] R. Hirota, "Exact solutions of the sine-Gordon equation for multiple collisions of solitons", J. Phys. Soc. Japan 33, 1459-1463 (1972).

[10] M. Jaworski and J. Zagrodzinski, "Positon and positon-like solutions of the Korteweg-de Vries and sine-Gordon equations", Chaos, Solitons \& Fractals 5, 2229-2233 (1995).

[11] D. J. Kaup, "Method for solving the sine-Gordon equation in laboratory coordinates", Stud. Appl. Math. 54, 165-179 (1975).

[12] V. A. Marchenko, Nonlinear Equations and Operator Algebras, Mathematics and Its Applications 17 (D. Reidel Publishing Company, Dordrecht 1988).

[13] V. B. Matveev and M. A. Salle, Darboux Transformations and Solitons, Springer Series in Nonlinear Dynamics (Springer-Verlag, Berlin 1991).

[14] V. B. Matveev, "Theory of Positons I", Preprint des Max-PlanckInstitutes für Metallforschung, Stuttgart (1992).

[15] V. B. Matveev, "Generalized Wronskian formula for solutions of the KdV equations: first applications", Physics Letters A 166, 205-208 (1992).

[16] V. B. Matveev, "Positon-positon and soliton-positon collisions: KdV case", Physics Letters A 166, 209-212 (1992).

[17] V. B. Matveev, "Asymptotics for the multipositon-soliton $\tau$ function of the Korteweg-de Vries equation and the supertransparency", J. Math. Phys. 35, 2955-2970 (1994). 
[18] S. Novikov, S. V. Manakov, L. P. Pitaevskii, and V. E. Zakharov, Theory of Solitons. The Inverse Scattering Method (Consultants Bureau, New York 1984).

[19] C. Pöppe, "Construction of solutions of the sine-Gordon equation by means of Fredholm determinants", Physica 9D, 103-139 (1983).

[20] G. Pólya and G. Szegö, Problems and Theorems in Analysis. Vol.II, Springer Study Edition (Springer-Verlag, Berlin 1976).

[21] C. Rasinariu, U. Sukhatme, and A. Khare, "Negaton and positon solutions of the KdV and mKdV hierarchy", J. Phys. A: Math. Gen. 29, 1803-1823 (1996).

[22] C. Schiebold, Funktionalanalytische Methoden bei der Behandlung von Solitonengleichungen (Thesis, Jena 1996).

[23] C. Schiebold, "An operator theoretic approach to the Toda lattice equation", Physica D 122, 37-61 (1998).

[24] P. C. Schuur, Asymptotic Analysis of Soliton Problems. An Inverse Scattering Approach, Lecture Notes in Mathematics 1232 (SpringerVerlag, Berlin 1986).

[25] J. Sylvester, "Sur l'equation en matrices $p x=x q$ ", C. R. Acad. Sci. Paris 99, 67-71 and 115-116 (1884).

[26] M. Toda, Nonlinear waves and solitons, Mathematics and Its Applications: Japanese Series (KTK Scientific Publishers, Tokyo 1989).

[27] V. E. Zakharov (Editor), What is Integrability? Springer Series in Nonlinear Dynamics (Springer-Verlag, Berlin 1991).

Fakultät für Mathematik und Informatik

Universität Jena

Ernst-Abbe-Platz 1-4

D-07743 Jena, Germany

E-mail: cornelia@minet.uni-jena.de

Recibido: 3 de Mayo de 2001

Revisado: 3 de Mayo de 2001 